US Army Corps

of Engineers $\mathrm{B}_{\circledast}$

Engineer Research and

Development Center

Military Engineering

Geospatial Research and Engineering

\title{
Infrasound Propagation in the Arctic
}

D. Keith Wilson, Vladimir E. Ostashev, Michael J. Shaw,

December 2021

Michael B. Muhlestein, John W. Weatherly, Michelle E.

Swearingen, and Sarah L. McComas
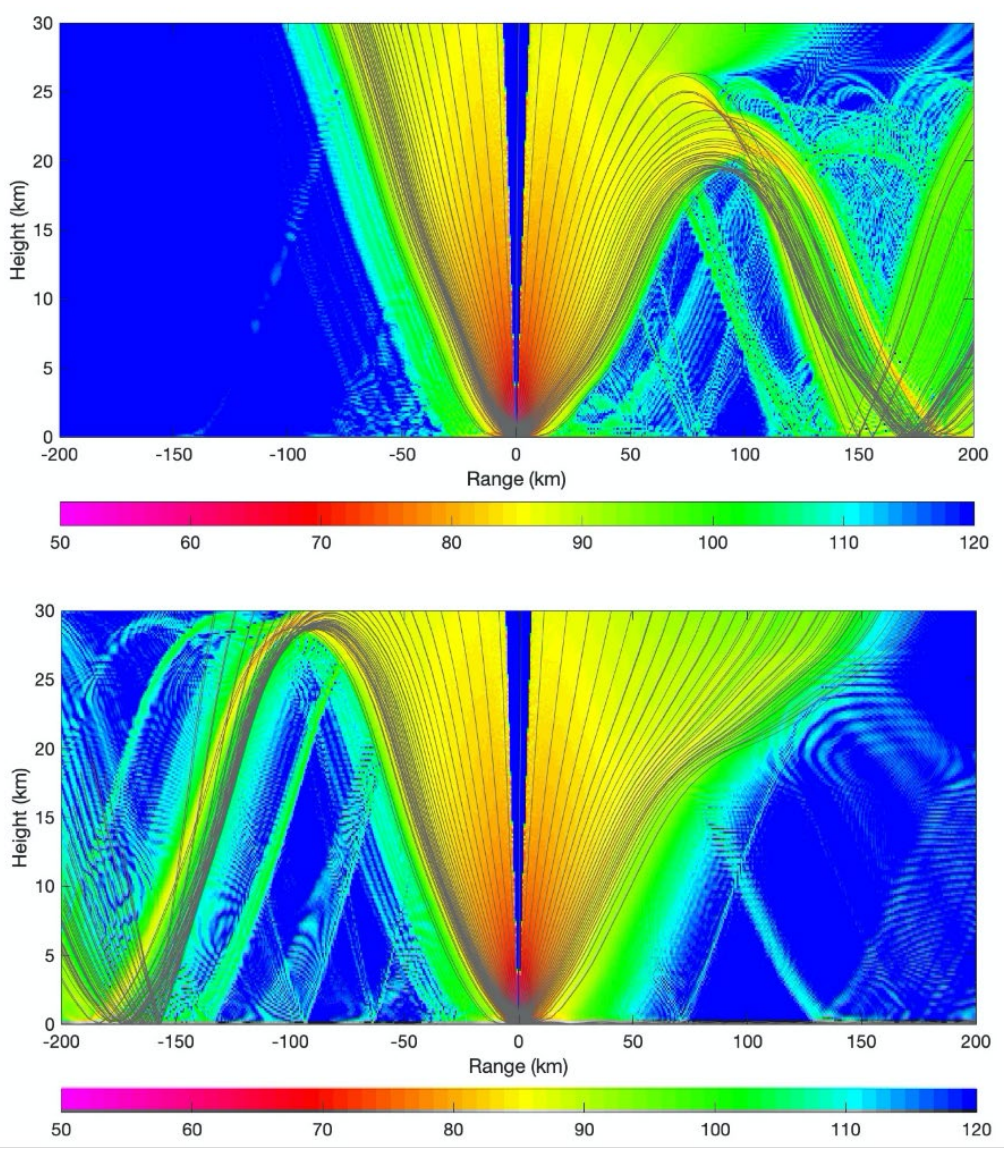

Approved for public release; distribution is unlimited. 
The U.S. Army Engineer Research and Development Center (ERDC) solves the nation's toughest engineering and environmental challenges. ERDC develops innovative solutions in civil and military engineering, geospatial sciences, water resources, and environmental sciences for the Army, the Department of Defense, civilian agencies, and our nation's public good. Find out more at www.erdc.usace.army.mil.

To search for other technical reports published by ERDC, visit the ERDC online library at https://erdclibrary.on.worldcat.org/v2. 


\section{Infrasound Propagation in the Arctic}

D. Keith Wilson, Vladimir E. Ostashev, Michael J. Shaw, Michael B. Muhlestein, and John W. Weatherly

U.S. Army Engineer Research and Development Center (ERDC)

Cold Regions Research and Engineering Laboratory (CRREL)

72 Lyme Road

Hanover, NH 03755-1290

Michelle E. Swearingen

Construction Engineering Research Laboratory (CERL)

U.S. Army Engineer Research and Development Center (ERDC)

2902 Newmark Drive

Champaign, IL 61822

Sarah L. McComas

Geotechnical and Structures Laboratory (GSL)

U.S. Army Engineer Research and Development Center (ERDC)

3909 Halls Ferry Road

Vicksburg, MS 39180-6199

Final Report

Approved for public release; distribution is unlimited.

Prepared for U.S. Army Engineer Research and Development Center Cold Regions Research and Engineering Laboratory 72 Lyme Road Hanover, NH 03755-1290

Under PE 0601102A/T22/02 


\section{Abstract}

This report summarizes results of the basic research project "Infrasound Propagation in the Arctic.” The scientific objective of this project was to provide a baseline understanding of the characteristic horizontal propagation distances, frequency dependencies, and conditions leading to enhanced propagation of infrasound in the Arctic region. The approach emphasized theory and numerical modeling as an initial step toward improving understanding of the basic phenomenology, and thus lay the foundation for productive experiments in the future. The modeling approach combined mesoscale numerical weather forecasts from the Polar Weather Research and Forecasting model with advanced acoustic propagation calculations. The project produced significant advances with regard to parabolic equation modeling of sound propagation in a windy atmosphere. For the polar low, interesting interactions with the stratosphere were found, which could possibly be used to provide early warning of strong stratospheric warming events (i.e., the polar vortex). The katabatic wind resulted in a very strong low-level duct, which, when combined with a highly reflective icy ground surface, leads to efficient long-distance propagation. This information is useful in devising strategies for positioning sensors to monitor environmental phenomena and human activities.

DISCLAIMER: The contents of this report are not to be used for advertising, publication, or promotional purposes. Citation of trade names does not constitute an official endorsement or approval of the use of such commercial products. All product names and trademarks cited are the property of their respective owners. The findings of this report are not to be construed as an official Department of the Army position unless so designated by other authorized documents.

DESTRUCTION NOTICE-Destroy by any method that will prevent disclosure of contents or reconstruction of the document. 


\section{Contents}

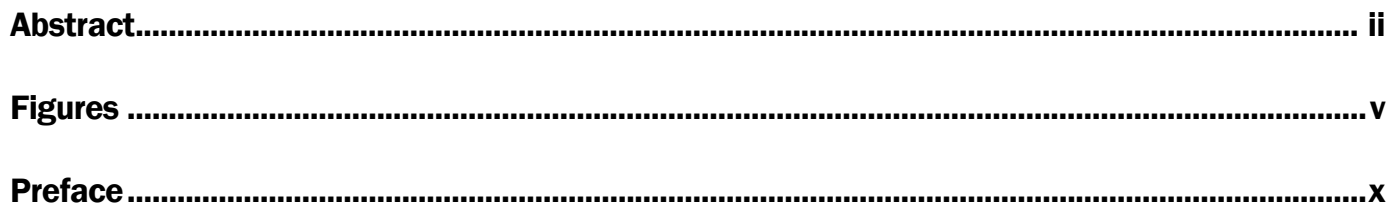

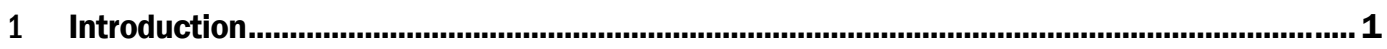

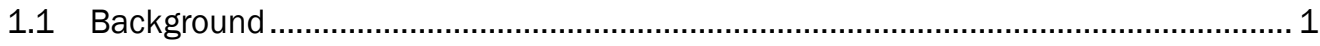

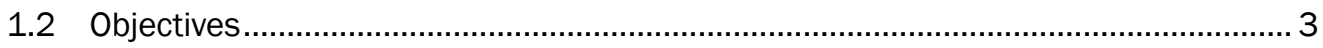

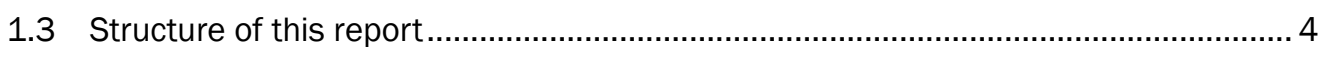

2 Project Overview and Accomplishments ............................................................................. 5

2.1 Narrative ........................................................................................................... 5

2.1.1 Wide-angle parabolic equation (WAPE) methods ..................................................... 5

2.1.2 Comparative study of propagation in Arctic and temperate conditions.......................... 6

2.1.3 High-resolution mesoscale modeling of Arctic weather coupled to acoustic

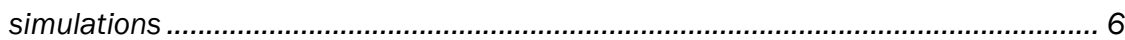

2.2 Publications.......................................................................................................... 7

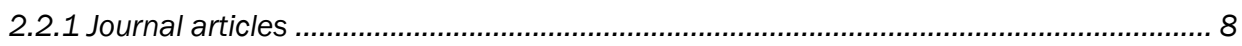

2.2.2 Conference presentations and proceedings.............................................................. 9

2.2.3 Significant unpublished presentations................................................................ 13

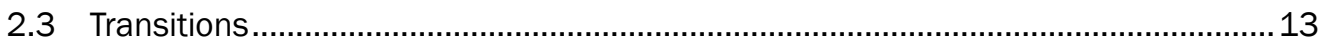

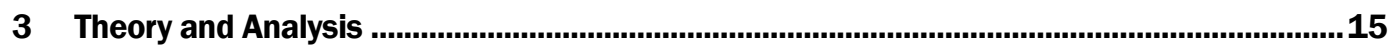

3.1 Background ...................................................................................................... 15

3.2 Extra-wide-angle parabolic equation (EWAPE) ...................................................... 15

3.3 Approximation for short wavelengths/large inhomogeneities .................................18

3.4 Padé series solution ......................................................................................... 20

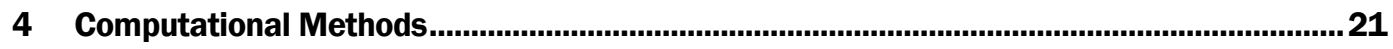

4.1 Numerical solution of the parabolic equation........................................................ 21

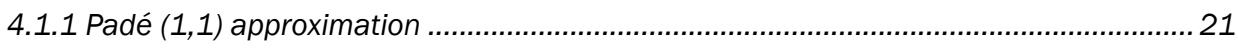

4.1.2 Padé $(2,2)$ approximation ................................................................................ 22

4.2 Polar Weather Research and Forecasting (PWRF) ........................................ 25

4.3 Interfacing PWRF to the parabolic equation .....................................................26

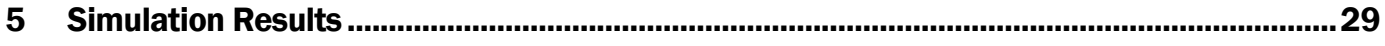

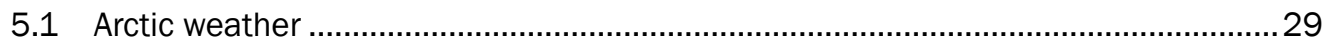

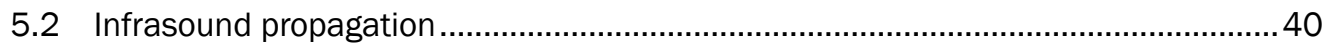

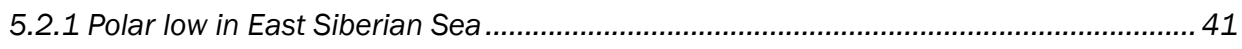

5.2.2 Katabatic wind in northern Greenland .................................................................... 44

5.3 Comparison of infrasound propagation at temperate and Arctic locations ..............46

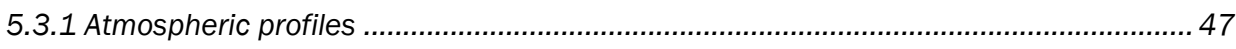

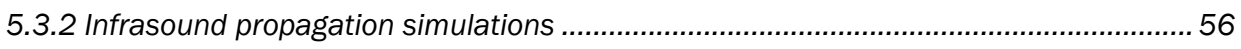

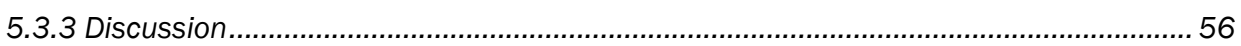




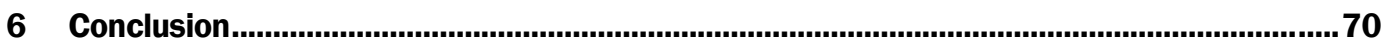

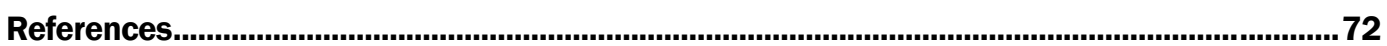

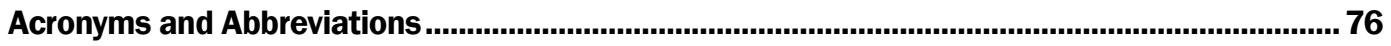

Report Documentation Page 


\section{Figures}

\section{Figures}

Figure 1. Normalized differences between the exact phase increment of a plane wave after propagation through the 3-D homogeneous slab and those calculated with various parabolic equations versus the angle relative to the propagation axis, $\theta_{0}$. For this calculation, the Mach number parallel to the propagation is $M x=0.025$, whereas the Mach number perpendicular to the propagation is $M \perp=0$. NAPE is the narrow-angle PE, the Padé approximations are wide-angle PEs, and the EWAPEs are the extra-wideangle PEs. The subscript "eff" indicates the effective sound-speed approximation. (The horizontal axis is truncated before $90^{\circ}$ because some of the solutions become infinite.)

Figure 2. Same as Figure 1, except that for this calculation, the Mach number parallel to the propagation is $M x=0$, whereas the Mach number perpendicular to the propagation is $M \perp=0.025$.

Figure 3. Comparison of basic narrow-angle PE (left), Green's function PE (center), and EWAPE (right) for propagation in a turbulent atmosphere.

Figure 4. The EASEE environmental abstraction layer (EnvironScenario) is constructed from a variety of data resources for terrain elevation, land cover, soils, and atmospheric forecasts and models. The environmental data are converted to the propagator media, which are then used for signal propagation modeling.

Figure 5. Data from the Climate Prediction Center (CPC) of the National Oceanic and Atmospheric Administration (NOAA) depicting major stratospheric warming during the winter of 2012-2013. Temperature at $50 \mathrm{mb}$ averaged from $65-90^{\circ} \mathrm{N}$, with minimum, maximum, and mean temperature for the period of record (1979-2008).

Figure 6. Geopotential height (roughly the height of the $50 \mathrm{mb}$ pressure surface) for the peak of the major SSW event on 6 January 2013 highlighting the typical bifurcation of the stratospheric vortex with localized (and zonally averaged) reversal of the zonal wind (in the west-east direction).

Figure $7.50 \mathrm{mb}$ surface temperatures on 6 January 2013 highlighting pronounced warm sectors that are tens of degrees Kelvin higher than normal for the date.......

Figure 8. Blue transect geolocating the wind and temperature cross section in Figure 9.

Figure 9. Zonal wind (solid and dashed black contours and labels) and air temperature at $\mathrm{OZ}$ on 6 January 2013 for latitude/longitude pairs along the $x$-axis of the figure. Note the warm perturbations and stratospheric jet reversal in the upper left of the figure......

Figure 10. The "Great Arctic Cyclone of 2012" as seen in the surface pressure field at $21 Z$ on 6 August 2012 exhibiting an intense low-pressure core.

Figure 11. A vertical cross section of the Great Arctic Cyclone of 2012 exhibiting strong jets (wind cores), temperature gradients, and a warm core or "eye." Color fill refers to zonal wind speed $(\mathrm{m} / \mathrm{s})$, and contour lines refer to air temperature (K).

Figure 12. A complex of polar lows off the coast of Norway at $12 Z$ on 19 November 
2015 as depicted through the surface pressure field. The yellow line represents a transect through the core of one polar low that is highlighted in a vertical cross section in the following figure.

Figure 13. A vertical cross section of the polar low in Figure 12, showing temperature and zonal wind. Note the occlusion, temperature inversions, and a lowlevel jet (LL) in the lower left of the figure.

Figure 14. Red transect near the sea ice edge to geolocate the LL via cross section on August 10, 2007.

Figure 15. Several jets (the blue, finger-like protrusions of high wind speed) in a vertical cross section near the sea ice edge, with a characteristic temperature inversion over and near the near-surface jet core.

Figure 16. A longitudinal view of wind and temperature inversions associated with the LU in Figure 15.

Figure 17. Vertical profiles for the East Siberian Sea, as extracted from the PWRF simulation at $\mathrm{OZ}$ on 6 January 2013 , at $73.372^{\circ}$ latitude and $150.555^{\circ}$ longitude. Height is $\mathrm{km}$ above ground (sea) level.

Figure 18. WAPE (Padé $(1,1)$ ) calculations for the East Siberian Sea polar low case, for a $5 \mathrm{Hertz}(\mathrm{Hz})$ source at $5 \mathrm{~m}$ height. In the top figure, westward propagation is to the left, eastward is to the right. In the bottom figure, southward propagation is to the left, northward to the right. The color scale represents transmission loss (TL) from $50 \mathrm{~dB}$ to $120 \mathrm{~dB}$. Gray lines are rays launched at $3^{\circ}$ increments.

Figure 19. Similar to Figure 18, except that the difference between the TLs predicted by the WAPE and NAPE calculations is shown. The color scale represents difference in TLs on a scale from $-20 \mathrm{~dB}$ to $20 \mathrm{~dB}$. Dashed lines are shown at angles of $25^{\circ}, 50^{\circ}$, and $80^{\circ}$ and relative to the horizontal. (Note that the horizontal and vertical scales differ so that the plotted angles of the lines differ from the physical angles.).

Figure 20. Close-up of the eastward propagation in the vicinity of a caustic. Top left is the NAPE calculation with rays calculated using the correct equations for a moving medium. Top right is the NAPE calculation with rays calculated using the ESSA. Bottom is the WAPE calculation with rays calculated using the correct equations for a moving medium. The location of the caustic is indicated by the white ellipses.

Figure 21. Vertical profiles for northern Greenland, as extracted from the PWRF simulation at $\mathrm{OZ}$ on $6 \mathrm{Jan} 2013$, at $77.592^{\circ}$ latitude and $-49.926^{\circ}$ longitude. Height is in $\mathrm{km}$ above ground level, which is at $2480 \mathrm{~m}$.

Figure 22. WAPE (Padé $(1,1)$ ) calculations for the northern Greenland katabatic wind case, for a $5 \mathrm{~Hz}$ source at $5 \mathrm{~m}$ height. See caption to Figure 18 for further explanation.

Figure 23. Radiosonde profiles for Danmarkshavn, Greenland, $12 Z$ March 2018. Shown are the sound speed (left), eastward (vx) wind component (center), and northward (vy) wind component (right). Each color is a different daily profile over the course of a single week. The thick black line is the mean profile for the week.

Figure 24. Radiosonde profiles for Fairbanks, AK, $12 Z$ March 2018. The profiles and colors are explained in Figure 23

Figure 25. Radiosonde profiles for Jackson, MS, $12 Z$ March 2018. The profiles and colors are explained in Figure 23. 
Figure 26. Radiosonde profiles for Danmarkshavn, Greenland, $12 Z$ June 2018. The profiles and colors are explained in Figure 23.

Figure 27. Radiosonde profiles for Fairbanks, AK, $12 Z$ June 2018. The profiles and colors are explained in Figure 23.

Figure 28. Radiosonde profiles for Jackson, MS, $12 Z$ June 2018. The profiles and colors are explained in Figure 23.

Figure 29. Radiosonde profiles for Danmarkshavn, Greenland, 12Z September 2018. The profiles and colors are explained in Figure 23.

Figure 30. Radiosonde profiles for Fairbanks, AK, $12 Z$ September 2018. The profiles and colors are explained in Figure 23.

Figure 31. Radiosonde profiles for Jackson, MS, $12 Z$ September 2018. The profiles and colors are explained in Figure 23.

Figure 32. Radiosonde profiles for Danmarkshavn, Greenland, 12Z December 2018. The profiles and colors are explained in Figure 23...

Figure 33. Radiosonde profiles for Fairbanks, AK, $12 Z$ December 2018. The profiles and colors are explained in Figure 23.

Figure 34. Radiosonde profiles for Jackson, MS, $12 Z$ December 2018. The profiles and colors are explained in Figure 23. 55

Figure 35. TL versus distance, 17-23 March 2018, 12Z, Danmarkshavn, Greenland. Thick black line represents the mean profile for the week, all other lines represent individual days. Top plot shows propagation to the west (left) and east (right). Bottom plot shows propagation to the south (left) and north (right). Frequency is $4 \mathrm{~Hz}$.

Figure 36. TL as a function of range and altitude, mean profile for the 17-23 March 2018 time period, 12Z, in Danmarkshavn, Greenland. Top plot shows propagation to the west (left) and east (right). Bottom plot shows propagation to the south (left) and north (right). Frequency is $4 \mathrm{~Hz}$.

Figure 37. TL versus distance, 17-23 March 2018, 12Z, Fairbanks, AK. Thick black line represents the mean profile for the week, all other lines represent individual days. Top plot shows propagation to the west (left) and east (right). Bottom plot shows propagation to the south (left) and north (right). Frequency is $4 \mathrm{~Hz}$.

Figure 38. TL as a function of range and altitude, mean profile for the 17-23 March 2018 time period, 12Z, in Fairbanks, AK. Top plot shows propagation to the west (left) and east (right). Bottom plot shows propagation to the south (left) and north (right). Frequency is $4 \mathrm{~Hz}$.

Figure 39. TL versus distance, 17-23 March 2018, 12Z, Jackson, MS. Thick black line represents the mean profile for the week, all other lines represent individual days. Top plot shows propagation to the west (left) and east (right). Bottom plot shows propagation to the south (left) and north (right). Frequency is $4 \mathrm{~Hz}$.

Figure 40. TL as a function of range and altitude, mean profile for the 17-23 March 2018 time period, 12Z, in Jackson, MS. Top plot shows propagation to the west (left) and east (right). Bottom plot shows propagation to the south (left) and north (right). Frequency is $4 \mathrm{~Hz}$.

Figure 41. TL versus distance, 18-24 June 2018, 12Z, Danmarkshavn, Greenland. Thick black line represents the mean profile for the week, all other lines represent individual days. Top plot shows propagation to the west (left) and east (right). Bottom plot shows propagation to the south (left) and 
north (right). Frequency is $4 \mathrm{~Hz}$.

Figure 42. TL as a function of range and altitude, mean profile for the 18-24 June 2018 time period, 12Z, in Danmarkshavn, Greenland. Top plot shows propagation to the west (left) and east (right). Bottom plot shows propagation to the south (left) and north (right). Frequency is $4 \mathrm{~Hz}$.

Figure 43. TL versus distance, 18-24 June 2018, 12Z, Fairbanks, AK. Thick black line represents the mean profile for the week, all other lines represent individual days. Top plot shows propagation to the west (left) and east (right). Bottom plot shows propagation to the south (left) and north (right). Frequency is $4 \mathrm{~Hz}$.

Figure 44. TL as a function of range and altitude, mean profile for the 18-24 June 2018 time period, 12Z, in Fairbanks, AK. Top plot shows propagation to the west (left) and east (right). Bottom plot shows propagation to the south (left) and north (right). Frequency is $4 \mathrm{~Hz}$.

Figure 45. TL versus distance, 18-24 June 2018, 12Z, Jackson, MS. Thick black line represents the mean profile for the week, all other lines represent individual days. Top plot shows propagation to the west (left) and east (right). Bottom plot shows propagation to the south (left) and north (right). Frequency is $4 \mathrm{~Hz}$.

Figure 46. TL as a function of range and altitude, mean profile for the 18-24 June 2018 time period, 12Z, in Jackson, MS. Top plot shows propagation to the west (left) and east (right). Bottom plot shows propagation to the south (left) and north (right). Frequency is $4 \mathrm{~Hz}$..

Figure 47. TL versus distance, 19-25 September 2018, 12Z, Danmarkshavn, Greenland. Thick black line represents the mean profile for the week, all other lines represent individual days. Top plot shows propagation to the west (left) and east (right). Bottom plot shows propagation to the south (left) and north (right). Frequency is $4 \mathrm{~Hz}$..

Figure 48. TL as a function of range and altitude, mean profile for the 19-25 September 2018 time period, 12Z, in Danmarkshavn, Greenland. Top plot shows propagation to the west (left) and east (right). Bottom plot shows propagation to the south (left) and north (right). Frequency is $4 \mathrm{~Hz}$.

Figure 49. TL versus distance, 19-25 September 2018, 12Z, Fairbanks, AK. Thick black line represents the mean profile for the week, all other lines represent individual days. Top plot shows propagation to the west (left) and east (right). Bottom plot shows propagation to the south (left) and north (right). Frequency is $4 \mathrm{~Hz}$.

Figure 50. TL as a function of range and altitude, mean profile for the 19-25 September 2018 time period, 12Z, in Fairbanks, AK. Top plot shows propagation to the west (left) and east (right). Bottom plot shows propagation to the south (left) and north (right). Frequency is $4 \mathrm{~Hz}$.

Figure 51. TL versus distance, 19-25 September 2018, 12Z, Jackson, MS. Thick black line represents the mean profile for the week, all other lines represent individual days. Top plot shows propagation to the west (left) and east (right). Bottom plot shows propagation to the south (left) and north (right). Frequency is $4 \mathrm{~Hz}$.

Figure 52. TL as a function of range and altitude, mean profile for the 19-25 September 2018 time period, 12Z, in Jackson, MS. Top plot shows propagation to the west (left) and east (right). Bottom plot shows propagation to the south (left) and north (right). Frequency is $4 \mathrm{~Hz}$. 
Figure 53. TL versus distance, 18-24 December 2018, 12Z, Danmarkshavn, Greenland. Thick black line represents the mean profile for the week, all other lines represent individual days. Top plot shows propagation to the west (left) and east (right). Bottom plot shows propagation to the south (left) and north (right). Frequency is $4 \mathrm{~Hz}$..

Figure 54. TL as a function of range and altitude, mean profile for the 18-24

December 2018 time period, 12Z, in Danmarkshavn, Greenland. Top plot shows propagation to the west (left) and east (right). Bottom plot shows propagation to the south (left) and north (right). Frequency is $4 \mathrm{~Hz}$.

Figure 55. TL versus distance, 18-24 December 2018, 12Z, Fairbanks, AK. Thick black line represents the mean profile for the week, all other lines represent individual days. Top plot shows propagation to the west (left) and east (right). Bottom plot shows propagation to the south (left) and north (right). Frequency is $4 \mathrm{~Hz}$.

Figure 56. TL as a function of range and altitude, mean profile for the 18-24 December 2018 time period, 12Z, in Fairbanks, AK. Top plot shows propagation to the west (left) and east (right). Bottom plot shows propagation to the south (left) and north (right). Frequency is $4 \mathrm{~Hz}$. 68

Figure 57. TL versus distance, 18-24 December 2018, 12Z, Jackson, MS. Thick black line represents the mean profile for the week, all other lines represent individual days. Top plot shows propagation to the west (left) and east (right). Bottom plot shows propagation to the south (left) and north (right). Frequency is $4 \mathrm{~Hz}$.

Figure 58. TL as a function of range and altitude, mean profile for the 18-24 December 2018 time period, 12Z, in Jackson, MS. Top plot shows propagation to the west (left) and east (right). Bottom plot shows propagation to the south (left) and north (right). Frequency is $4 \mathrm{~Hz}$. 


\section{Preface}

This study was conducted for the Assistant Secretary of the Army for Acquisition, Logistics, and Technology under the U.S. Army Engineer Research and Development Center (ERDC) 6.1 Geospatial Research and Engineering (GRE) Basic Research Program, PE 0601102A/T24, Work Item B78BoJ, and the 6.1 Military Engineering (ME) Basic Research Program, PE 0601102A/T22/o2, Work Item 3CL2G6.

The work was performed by the Signature Physics Branch, CEERD-RRD, of the Research \& Engineering Division, CEERD-RR, Engineer Research and Development Center, Cold Regions Research and Engineering Laboratory (ERDC-CRREL). At the time of publication, Dr. M. Andrew Niccolai was Chief, CEERD-RRD, Dr. George Calfas was Acting Chief, CEERD-RR, and Dr. Robert E. Davis was Technical Director for Geospatial Research and Engineering/Military Engineering. The Deputy Director of ERDC-CRREL was Mr. David B. Ringelberg, and the Director was Dr. Joseph L. Corriveau.

COL Teresa A. Schlosser was Commander of ERDC, and Dr. David W. Pittman was the Director. 


\section{Introduction}

\subsection{Background}

Due to changing climate and the reemerging threat posed by near-peer adversaries, the Arctic and sub-Arctic are becoming increasingly contested militarily and economically (Center for Strategic and International Studies 2020). By better understanding the impacts of this unique environment on infrasound, infrasound from military and industrial activities can potentially be sensed over long distances (Gibbons and Ringdal 2010). Infrasound also presents opportunities for monitoring environmental phenomena related to climate change. Glacier destruction, for example, has been shown to produce infrasonic emissions (Asming et al. 2016). It is also plausible that atmospheric infrasound can be used to monitor largescale cracking events and collisions in ice floes, as is done with underwater sound (Farmer and Xie 1989). Furthermore, new remote sensing techniques could possibly be developed for probing the upper atmosphere with infrasound to provide advance warning of sudden stratospheric warming (SSW) events, which are the precursor of the polar vortex.

Phenomena particular to the polar regions-including circumpolar wind patterns, low-level jets above strongly stable layers, strong temperature and humidity gradients (particularly near the land and ocean surfaces), and density currents-combine with snow and ice at northern latitudes to produce unique propagation effects that are not ordinarily observed at temperate and tropical latitudes. The circumpolar wind, for example, could cause unusual asymmetries in propagation. Extremely strong temperature inversions, when coupled with acoustically hard surfaces such as ice and water, enable propagation over long distances with minimal transmission losses. Latent heat fluxes over sea ice and snow are potentially strong enough to produce significant refraction by humidity gradients, which is rarely observed at lower latitudes.

While underwater sound propagation in the Arctic has been extensively studied (e.g., Gavrilov and Mikhalevsky 2006, and references therein) and remains an important topic for research at the present time, sound propagation in the Arctic atmosphere has received considerably less attention. Previous research on infrasound propagation in the Arctic has generally focused on propagation over global scales, as pertinent to 
monitoring nuclear tests and other very large explosions (Wilson et al. 2005; Olson and Szuberla 2005; Szuberla and Olson 2004). The basic refraction phenomena involving the upper atmosphere are largely well understood. In particular, the stratospheric temperature inversion and jet stream lead to zones of audibility and inaudibility at long distances (e.g., Pierce 1981; Drob et al. 2003; Ostashev and Wilson 2015). In fact, the presence of such "abnormal" audibility zones was one of the primary phenomena used to infer the presence of the stratospheric temperature inversion and jet stream over a century ago. (See, for example, the discussion in Pierce [1981].)

At the regional and near-regional scale (distances up to roughly 1000 kilometers [km]), infrasound research has focused primarily on the temperate and equatorial latitudes (McKenna et al. 2008; McKenna et al. 2012), due partly to the challenges of locating stations in the polar regions and the limited activity in these regions. A notable exception is Ringdal et al. (2010), who deployed regional seismic and infrasound arrays in northern Europe. Gibbons and Ringdal (2010) reported that military and industrial activities in Finland and Northwest Russia generate infrasound, which was detectable regionally; 350 events were detected over an 8-year period.

Many propagation phenomena are likely unique or unusually strong in the Arctic. Most obvious, perhaps, is the impact of very low temperatures and specific humidity. Andreas (1988) showed that the Arctic climate has very complex impacts on the optical index of refraction; however, impacts on acoustic index of refraction and attenuation have not been closely examined. Some insight into the behavior of acoustic attenuation in very cold air is provided by studies in northern temperate climates (Wilson and Thomson 1991; Okada et al. 2016), which found that attenuation during the winter is substantially higher and varies dramatically with specific humidity.

Another Arctic phenomenon that is likely important to sound propagation is the presence of extremely strong surface-based stable layers (temperature inversions) capped by low-level jets. Some insights into this phenomenon can be gained from previous investigations of low-frequency sound propagation through continental boundary layers in nighttime, fair weather conditions (e.g., Wilson et al. 2003; Waxler et al. 2006). Although the acoustic wavelengths and the scale of the pertinent atmospheric 
structure are considerably smaller, such features as strong surface-based temperature inversions and strong wind shear aloft are present. While strongly stable atmospheric conditions normally appear very calm to a casual observer, they produce very strong, dynamic scattering of sound, as illustrated by the parabolic equation (PE) calculations shown in Wilson et al. (2003).

\subsection{Objectives}

This report summarizes results of the two-year basic research project "Infrasound Propagation in the Arctic." The primary objective of this project is to better understand the impacts of land, sea-surface, and the atmosphere of the Arctic region on sound propagation. The focus is on infrasound $(<20 \mathrm{~Hz})$ propagation over horizontal distances of several thousand $\mathrm{km}$ and less, although the research also has pertinence to lowfrequency sound in the audible range $(20 \mathrm{~Hz}-200 \mathrm{~Hz})$.

In particular, we hypothesize that atmospheric phenomena particular to the polar regions, which include wind patterns such as the polar vortex, low-level jets above strongly stable layers, strong temperature and humidity gradients (particularly near the land and ocean surfaces, but also at other sharp interfaces between air masses), and density currents, have unique impacts on infrasonic and low-frequency propagation that are not ordinarily observed at temperate and tropical latitudes. This study endeavored to provide a baseline understanding of conditions leading to enhanced propagation in the Arctic, as well as what frequency ranges, horizontal distances, and strategies for sensor positioning will be most useful for monitoring activities in the Arctic and for remote sensing of the upper atmosphere.

This project emphasized theory and numerical modeling as the initial step toward improving understanding of the basic phenomenology of infrasonic propagation in the Arctic. With the advances in numerical weather and sound propagation modeling made over the past several decades, simulations can attain a very high level of realism and lay the ground work for productive experiments in the future, which involve substantial logistic challenges and expense in polar regions. The modeling approach for this project combined mesoscale ${ }^{*}$ numerical weather forecasts and advanced

\footnotetext{
* In atmospheric sciences, mesoscale typically refers to phenomena with a spatial scale between $2 \mathrm{~km}$ and $2000 \mathrm{~km}$. Smaller phenomena are termed microscale, whereas larger phenomena are synoptic scale.
} 
acoustic propagation techniques suitable for a windy atmosphere with temperature and humidity stratification.

From an acoustical modeling perspective, propagation in polar regions presents formidable challenges with regard to high wind speeds and strong wind shear, strong temperature inversions and humidity gradients, and parameterizations of processes involving snow and ice. Therefore, a substantial portion of the project effort involved formulating correct theoretical equations suitable to propagation calculations in a windy atmosphere and numerical methods that could solve these equations efficiently and accurately. Extensive attention also had to be paid to properly interfacing the mesoscale numerical weather forecasts to the acoustic propagation calculations since the former is performed on a 3-D grid in polar stereographic coordinates, whereas the latter assumes a Cartesian grid.

\subsection{Structure of this report}

Section 2 provides an overview of the project and its primary accomplishments, including publications. Section 3 summarizes the theory formulated for sound propagation in a moving medium, which is needed to simulate propagation in high winds and shear as occurs in Arctic environments. Next, in Section 4, new methods are described for solving the propagation equations, which enable efficient, accurate infrasound propagation calculations over long distances. Lastly, in Section 5, the simulations of infrasound propagation in the Arctic are detailed, which suggest a number of interesting propagation effects related to polar lows, katabatic winds, and persistent Arctic temperature inversions. 


\section{Project Overview and Accomplishments}

\subsection{Narrative}

This project had three primary components. The following subsections summarize these components and the progress made in addressing them.

\subsubsection{Wide-angle parabolic equation (WAPE) methods}

Narrow-angle parabolic equations (NAPEs) have been the most popular numerical methodology for outdoor sound propagation calculations. They are suitable for propagation angles up to $15^{\circ}-20^{\circ}$ off the horizontal axis (i.e., propagation that is slanted at angles up to $15^{\circ}-20^{\circ}$ above the horizon). However, many practical long-range sound propagation problems involve refraction and scattering from elevated layers such that these angular limitations of NAPEs are exceeded. Wide-angle parabolic equations (WAPEs) can overcome this limitation; depending on the formulation, they are typically valid to angles of $40^{\circ}-45^{\circ}$. However, numerically feasible WAPEs that properly account for wind in the atmosphere or currents in the ocean were unavailable prior to this project.

To address the need for an accurate and computationally practical WAPE, we devised a fundamentally new approach that takes as the starting point the correct general equation set for sound propagation in a moving medium, developed by Ostashev and Wilson (2015), as approximated to sound-speed and wind velocity variations, which are relatively large compared to the wavelength. A new class of PEs called EWAPEs (extrawide-angle parabolic equations) was then derived. The EWAPEs are described in a journal article published in the Journal of the Acoustical Society of America (Ostashev et al. 2019). That article shows how other PEs in the literature, for both moving and nonmoving media, are special cases of the EWAPEs and compares calculation errors among the various methods. Pros and cons of the various approaches are discussed, and some numerical examples are provided.

Next, an efficient solution method was formulated as described in a conference paper for the International Congress on Acoustics (ICA) (Wilson et al. 2019) and in a journal article (Ostashev et al. 2020). This solution involved deriving a WAPE from the EWAPE using a Padé $(1,1)$ approximation, which is valid for low Mach numbers and angles up to 
roughly $40^{\circ}$. This new WAPE can be conveniently implemented with relatively small changes to conventional (narrow-angle Crank-Nicholson) $\mathrm{PE}$ codes, yet is accurate at wider angles and provides correct results for a moving atmosphere. We have also derived a Padé $(2,2)$ approximation, which is valid for angles of roughly $60^{\circ}$ and hence provides a suitable solution for nearly any practical problem involving infrasound propagation. However, implementation of this new Padé $(2,2)$ approximation has not yet been undertaken.

The PE modeling for this project significantly advanced the state of the art for sound propagation modeling in a moving medium. The objective was met for formulating viable methods for infrasound and low-frequency sound propagation through an inhomogeneous atmosphere; these methods provide accurate solutions for high wind speeds and shear for distances out to hundreds of kilometers.

\subsubsection{Comparative study of propagation in Arctic and temperate conditions}

This component of the project contrasted the impacts of the Arctic environment on infrasound propagation with those at temperate latitudes. The comparisons were based on atmospheric profiles collected in Vicksburg, Mississippi; Fairbanks, Alaska; and Danmarkshavn, Greenland. Results were originally presented at a meeting of the Acoustical Society of America and are also presented in more detail in Section 5.3 of this report.

\subsubsection{High-resolution mesoscale modeling of Arctic weather coupled to acoustic simulations}

As input to the infrasound propagation calculations with the WAPE methods described above, we chose to use the Weather Research and Forecasting (WRF) model, specifically the Arctic System Reanalysis (ASRv2) (Bromwich et al. 2018) utilizing the polar adaptation of WRF, called Polar WRF or PWRF. PWRF includes sea-ice surface, ice-cloud microphysics, and other modifications to the standard WRF release. ASRv2 provides time-varying, 3-D atmospheric fields. One of the practical challenges of the project was coding an interface that drives the 3-D sound propagation calculations with the 3-D atmospheric forecast data. To do this, we leveraged the ERDC EASEE (Environmental Awareness for Sensor and Emitter Employment) software, which enables full 3-D environmental 
variability and resampling to arbitrary geographic coordinate projections. The transformation of the weather model predictions from polar stereographic coordinates, to the cylindrical coordinate system for the acoustic calculations, was completed.

The importance of 3-D propagation effects was demonstrated in the first project year for a heterogeneous domain, namely the Chesapeake Bay region, and published in an SPIE conference paper (Wilson et al. 2018). In the second project year, we used data from PWRF to model propagation in the vicinity of a polar low in the East Siberian Sea, and with a katabatic wind (a type of density current) in northern Greenland using data from the PWRF model. The polar low exhibits interesting refractive returns from the stratosphere, which could be useful for detecting SSW events, the precursor to the polar vortex. The katabatic flow creates long-range surface ducting, with little attenuation of the sound energy.

\subsection{Publications}

This section provides a complete listing of the publications for this project, along with the abstracts from those publications.

Two journal articles had been accepted for publication at the time of this report. The first, Ostashev et al. (2019), derives a new class of PEs, called EWAPEs. All other PEs in the literature, for both moving and nonmoving media, are shown to be special cases of the EWAPEs. The paper describes many alternative approaches to deriving and implementing PEs, and compares calculation errors among the various methods. The second, Ostashev et al. (2020), examines various formulations of the EWAPEs and approximations to WAPEs in a moving medium with high Mach number. Numerical methods are derived that are shown to provide accurate solutions for sound propagation in a windy atmosphere. This publication provides, for the first time, efficient and accurate PE methods in a moving medium.

One remaining journal article is planned, which will describe the Arctic weather modeling and utilization of the new PE methods to simulate the impacts of the Arctic environment on infrasound propagation.

Six conference presentations were also made-four at Acoustical Society of America meetings, one at the SPIE Defense and Commercial Sensing Symposium, and an invited international presentation at the International 
Congress on Acoustics. A presentation was also made at the Cold Regions Science and Engineering Workshop, which was held at the U.S. Army Cold Regions Research and Engineering Laboratory (CRREL) in Hanover, New Hampshire. A demonstration of the infrasound prediction software was made at the Army Arctic S\&T Workshop, which was also held at CRREL.

\subsubsection{Journal articles}

Ostashev, V. E., M. B. Muhlestein, and D. K. Wilson. 2019. "Extra-WideAngle Parabolic Equations in Motionless and Moving Media." Journal of the Acoustical Society of America 145: 1031-47. doi:10.1121/1.5091011.

Abstract: Wide-angle parabolic equations (WAPEs) play an important role in physics. They are derived by an expansion of a square-root pseudodifferential operator in one-way wave equations and then solved by finitedifference techniques. In the present paper, a different approach is suggested. The starting point is an extra-wide-angle parabolic equation (EWAPE) valid for small variations of the refractive index of a medium. This equation is written in an integral form, solved by a perturbation technique, and transformed to the spectral domain. The resulting splitstep spectral algorithm for the EWAPE accounts for the propagation angles up to $90^{\circ}$ with respect to the nominal direction. This EWAPE is also generalized to large variations in the refractive index. It is shown that WAPEs known in the literature are particular cases of the two EWAPEs. This provides an alternative derivation of the WAPEs, enables a better understanding of the underlying physics and ranges of their applicability, and opens an opportunity for innovative algorithms. Sound propagation in both motionless and moving media is considered. The split-step spectral algorithm is particularly useful in the latter case since complicated partial derivatives of the sound pressure and medium velocity reduce to wave vectors (essentially, propagation angles) in the spectral domain.

Ostashev, V. E., D. K. Wilson, and M. B. Muhlestein. 2020. "Wave and Extra-Wide-Angle Parabolic Equations for Sound Propagation in a Moving Atmosphere." Journal of the Acoustical Society of America 147: 3969-84. doi:10.1121/10.0001397.

Abstract: The narrow-angle parabolic equation (NAPE), with the effective sound speed approximation (ESSA), is widely used for sound and 
infrasound propagation in a moving medium such as the atmosphere. However, it is valid only for angles less than $20^{\circ}$ with respect to the nominal propagation direction. In this paper, the wave equation and extrawide-angle parabolic equation (EWAPE) for high-frequency (shortwavelength) sound waves in a moving medium with arbitrary Mach numbers are derived without the ESSA. For relatively smooth variations in the medium velocity, the EWAPE is valid for propagation angles up to $90^{\circ}$. Using the Padé $(n, n)$ series expansion and narrow-angle approximation, the EWAPE is reduced to the wide-angle parabolic equation (WAPE) and NAPE. Versions of these equations are then formulated for low Mach numbers, which is the case usually considered in the literature. The phase errors pertinent to the equations considered are studied. It is shown that the equations for low Mach numbers and the WAPE with the ESSA are applicable only under rather restrictive conditions on the medium velocity. An effective numerical implementation of the WAPE for arbitrary Mach numbers in the Padé $(1,1)$ approximation is developed and applied to sound propagation in the atmosphere.

Wilson, D. K., M. J. Shaw, V. E. Ostashev, M. B. Muhlestein, R. E. Alter, M. E. Swearingen, S. L. McComas, and J. W. Weatherly. 2021. "Numerical Modeling of Mesoscale Infrasound Propagation in the Arctic." Submitted to Journal of the Acoustical Society of America (special issue on Arctic Ocean Acoustics).

\subsubsection{Conference presentations and proceedings}

Wilson, D. K., D. J. Breton, R. E. Alter, L. E. Waldrop, W. M. Barnes, M. B. Muhlestein, V. E. Ostashev. 2018. "Modeling RF and Acoustic Signal Propagation in Complex Environments." Ground/Air Multisensor Interoperability, Integration, and Networking for Persistent ISR IX, SPIE Defense and Commercial Sensing, Orlando, 15-19 April 2018, paper 1063519. doi:10.1117/12.2311592 (presentation and paper).

A computational framework is described for modeling acoustic and radiofrequency (RF) signal propagation in complex environments, such as urban, mountainous, and forested terrain. In such environments, the influences of three-dimensional atmospheric fields and terrain variations must be addressed. The approach described here involves creation of a full environmental data representation (abstraction layer), which can be initialized with many different environmental data resources, including 
weather forecasts, digital terrain elevations, land cover types, and soil properties. The environmental representation is then converted into the parameters needed for particular signal modalities and classes of propagation algorithms. In this manner, execution of the signal propagation calculations is isolated from the sources of environmental data so that all models will function with all types of environmental data. The formulation of the acoustic (infrasound and audible) and RF (VHF/UHF/SHF) feature spaces is also described. Example calculations involving infrasound propagation with $3-\mathrm{D}$ weather fields and $\mathrm{RF}$ propagation in mountainous terrain are provided.

Muhlestein, M. B., V. E. Ostashev, and D. K. Wilson. 2018. “A Green's Function Parabolic Equation Description of Infrasound Propagation in an Inhomogeneous and Moving Atmosphere." Acoustical Society of America, Minneapolis, 7-11 May 2018. doi:10.1121/1.5035677 (presentation and abstract).

Abstract: Accurate atmospheric infrasound propagation models must account for variations in local sound speed and ambient flow. This paper describes and demonstrates a method to extend the Green's function parabolic equation (GFPE) to account for 3-D high Mach number ambient flow in addition to an inhomogeneous atmosphere. Predictions of infrasonic propagation using the resulting GFPE model are then compared with predictions using other models.

Alter, R. E., M. E. Swearingen, and D. K. Wilson. 2018. "Determining Essential Scales and Associated Uncertainties for Regional Atmospheric Infrasound Propagation by Incorporating Three-Dimensional Weather Model Forecasts." Acoustical Society of America, Minneapolis, 7-11 May 2018. doi:10.1121/1.5035926 (presentation and abstract).

Abstract: Understanding infrasound propagation is important for geophysical and military applications. Infrasound signatures can be detected from larger sources such as nuclear detonations and from smaller sources such as bridges, dams, and buildings. Infrastructure sources produce signals of lower amplitude, leading to more regional (up to 150 $\mathrm{km}$ ) propagation. However, current methods for calculating regional infrasound propagation involve assumptions about the atmosphere, such as horizontal homogeneity, that deviate from more realistic environmental conditions and decrease the accuracy of the infrasound predictions. To 
remedy this issue, we have interfaced three-dimensional forecasts from the Weather Research and Forecasting (WRF) meteorological model with range-dependent parabolic equation propagation models. To test the improvement of infrasound propagation predictions with more realistic weather data, we conducted sensitivity studies with different propagation ranges and horizontal resolutions and compared them to predictions using simplified meteorological parameters. This allowed identification of the scales most ideal for resolving regional infrasound propagation given the limitations of WRF's spatiotemporal resolutions. Additionally, we present results on quantifying uncertainty in these infrasound simulations by using multiple realizations of WRF forecasts to generate a spread of possible outcomes. Finally, we compare the simulated results to experimental data.

Swearingen, M. E., S. L. McComas, D. K. Wilson, and V. E. Ostashev. 2019. "Similarities and Differences in Infrasound Propagation Effects between Arctic and Temperate Environments." Acoustical Society of America, Louisville, 13-17 MAY 2019. doi:10.1121/15101891 (presentation and abstract).

Abstract: Meteorological conditions in an arctic environment differ significantly from those in a temperate environment. Atmospheric phenomena particular to polar regions, including wind patterns such as the polar vortex and low-level jets above strongly stable layers, strong temperature and humidity gradients, and density currents, could have unique impacts on infrasound propagation that are not observed in temperate locations. In this study, parabolic-equation simulations of sound propagation are performed using measured meteorological conditions for summer and winter conditions in temperate and arctic locations. The similarities and differences in environmental conditions between these two locations and their relative impact on the predicted transmission loss are examined. For summer conditions, a comparison to measured data from explosive sources is performed for both temperate and arctic locations.

Wilson, D. K., M. B. Muhlestein, V. E. Ostashev, M. J. Shaw, M. E. Swearingen, and S. L. McComas. 2019. "Solution of Wide-Angle Parabolic Equations for Long-Range Sound Propagation in a Moving Medium." Proceedings of the International Congress on Acoustics, Aachen, Germany, 
9-13 September 2019 (invited international conference presentation and paper).

Abstract: Narrow-angle parabolic equations (NAPEs), which are widely used for outdoor sound propagation, are suitable for propagation angles up to $15^{\circ}-20^{\circ}$ off the nominal propagation axis (generally the horizontal direction). Wide-angle parabolic equations (WAPEs) are needed to accurately solve problems involving refraction and scattering from elevated layers at larger angles. However, it is challenging to formulate WAPEs that are numerically feasible to solve yet properly account for motion in the propagation medium (e.g., wind in the atmosphere). As a starting point, this paper considers an extra-wide-angle parabolic equation (EWAPE) for moving media that is valid for propagation angles up to $90^{\circ}$. Applying a Padé $(1,1)$ approximation to the EWAPE, a new WAPE, valid for low Mach numbers and angles up to roughly $40^{\circ}$, is then derived. The resulting equation is generally suitable for long-range sound propagation in a windy atmosphere. As an example, calculations are given here for infrasound in the Arctic. The NAPE and WAPE solutions are compared, and the latter is found to be in better agreement with predictions for caustic locations based on ray tracing for a windy atmosphere.

Ostashev, V. E., D. K. Wilson, M. B. Muhlestein, M. J. Shaw, M. E. Swearingen, and S. L. McComas. 2019. "Extra-Wide-Angle Parabolic Equation for Wave Propagation in Inhomogeneous Media." Acoustical Society of America, San Diego, 2-6 December 2019 (presentation and abstract).

Abstract: To describe wave propagation at large angles with respect to a nominal direction, wide-angle parabolic equations (WAPEs) have been widely used in atmospheric and ocean acoustics, geophysics, electromagnetic wave propagation, and other fields of physics. This paper considers an application of an extra-wide-angle parabolic equation (EWAPE) for such problems. For small variations of the refractive index of a medium, the EWAPE describes wave propagation up to 90 degrees with respect to the nominal direction and is more general than the WAPEs used in the literature. The EWAPE can be written in an integral form or as a pseudo-differential equation and may be solved by a split-step spectral algorithm or the Padé series expansions of the pseudo-differential operators. The EWAPE is also generalized to large variations in the refractive index, sound propagation above an impedance boundary, and a 
moving medium with small or large Mach numbers. For sound propagation in a moving medium, the EWAPE enables derivation of new WAPEs, which are accurate and simpler to implement than those currently available in the literature. Numerical examples illustrating the application of these new WAPEs to sound propagation in a moving atmosphere are presented.

\subsubsection{Significant unpublished presentations}

Wilson, D. K., M. J. Shaw, J. W. Weatherly, V. E. Ostashev, M. B. Muhlestein, M. E. Swearingen, and S. L. McComas. 2019. "Infrasound Propagation in the Arctic.” Cold Regions Science and Engineering Workshop, U.S. Army Cold Regions Research and Engineering Laboratory, Hanover, NH, 19-20 March 2019.

Waldrop, L. E., J. J. Gagnon, and D. K. Wilson. 2018. "Environmental Awareness for Sensor and Emitter Employment (EASEE).” Army Arctic S\&T Workshop, U.S. Army Cold Regions Research and Engineering Laboratory, Hanover, NH, 16-18 May 2018. (Demonstration of calculations of sound propagation in the Arctic and impact on air vehicle detection.)

\subsection{Transitions}

The capabilities for interfacing the parabolic equation sound propagation calculations with numerical weather forecast data from the WRF and PWRF models was built into the EASEE software (Wilson and Yamamoto 2014; Wilson et al. 2020). EASEE, which is used to model diverse problems involving signal detection and sensor performance, has been transitioned to multiple intelligence agencies and all five U.S. Department of Defense (DoD) services.

The new modeling capabilities developed for this project provide a foundation for improved Arctic domain awareness, for which vast areas must be efficiently monitored for military activity, including growing threats such as cruise missiles and unmanned aerial systems. Potential customer and partner relationships include the U.S. European Command (EUCOM), U.S. Northern Command (NORTHCOM), and the North American Aerospace Defense Command (NORAD). The research is relevant to the U.S. Army Modernization Priorities for Air and Missile 
Defense, Long-Range Precision Fires, and Network $\mathrm{C}_{3} \mathrm{I}$ (Command, Control, Communications, and Intelligence). 


\section{Theory and Analysis}

\subsection{Background}

NAPEs are widely used to calculate the impacts of refractive gradients and ground interactions on outdoor sound propagation (Gilbert and White 1989; West et al. 1992). They are suitable for propagation angles up to $15^{\circ}-20^{\circ}$ off the nominal propagation axis, which is typically taken to be the horizontal direction in the vertical plane containing the source and receiver. NAPEs usually employ the ESSA, in which the sound speed is replaced by the sum of the actual sound speed and the component of the wind speed along the nominal axis. The ESSA can be derived as a consequence of the narrow-angle approximation (Ostashev and Wilson 2015).

Many practical long-range sound propagation problems, however, involve refraction and scattering from elevated layers that exceed the $15^{\circ}-20^{\circ}$ limitation of NAPEs. WAPES are needed to accurately solve such problems (Ostashev et al. 1997; Blanc-Benon et al. 2001; Ostashev et al. 2002; Lingevitch et al. 2002). However, because the ESSA is inapplicable at wide angles, it is challenging to formulate WAPEs that are numerically feasible to solve yet properly account for motion in the propagation medium.

To address the need for an accurate and computationally practical WAPE, we formulated an entirely new approach to deriving parabolic equations. The starting point is the derivation of a family of EWAPEs for moving media, which account for propagation angles up to $90^{\circ}$ with respect to the nominal propagation direction. A WAPE is then derived from the EWAPE using a Padé approximation. Solution of this WAPE is then shown to be a straightforward extension of the conventional Crank-Nicholson method for solving the NAPE, while involving no significant additional computational burden. The key aspects of this development are described in the remainder of this chapter.

\subsection{EWAPE}

WAPEs play an important role in many fields, including atmospheric and ocean acoustics, geophysics, and electromagnetic wave propagation. They are usually derived from one-way wave equations, which involve a square- 
root pseudo-differential operator. This operator is then typically approximated by a series and solved by finite-difference techniques.

For the present project, we formulated a new approach to generalizing parabolic equations, which is described fully in Ostashev et al. (2019). This approach involved deriving the previously mentioned EWAPE, which is generally valid for small variations of the refractive index of a medium. Sound propagation in both motionless and moving media was examined. For small variations of the refractive index of a medium, the EWAPE describes wave propagation up to $90^{\circ}$ with respect to the nominal axis. The EWAPE can be written in an integral form or as a pseudo-differential equation and may be solved by a split-step spectral algorithm or the Padé series expansions of the pseudo-differential operators.

It was shown that WAPEs known in the literature are all particular cases of the two versions of EWAPEs derived in Ostashev et al. (2019). This approach provides an alternative pathway to deriving WAPEs, which enables a better understanding of the underlying physics and ranges of their applicability. The EWAPE was also generalized to large variations in the refractive index, to sound propagation above an impedance boundary, and to a moving medium with small or large Mach numbers. For sound propagation in a moving medium, the EWAPE enables derivation of new WAPEs that are accurate yet simpler to implement than those currently available in the literature.

For solving the full EWAPE, we employed a split-step spectral algorithm. The spectral approach is particularly useful in the present context since complicated partial derivatives of the medium density and velocity reduce to wave vectors (essentially, propagation angles) in the spectral domain.

Figure 1 and Figure 2 show phase errors associated with various parabolic approximations. The figures are, respectively, for flow along the propagation axis and perpendicular to it. The objective is for the phase error to be small out to as wide an angle as possible. The conventional NAPE has substantial phase errors starting at $20^{\circ}$, whereas the Padé $(1,1)$ approximation (the most widely used type of WAPE) has substantial phase errors starting at $35^{\circ}$. 
Figure 1. Normalized differences between the exact phase increment of a plane wave after propagation through the 3-D homogeneous slab and those calculated with various parabolic equations versus the angle relative to the propagation axis, $\boldsymbol{\theta}_{\mathbf{0}}$. For this calculation, the Mach number parallel to the propagation is $\boldsymbol{M}_{\boldsymbol{x}}=\mathbf{0 . 0 2 5}$, whereas the Mach number perpendicular to the propagation is $M_{\perp}=0$. NAPE is the narrow-angle PE, the Padé approximations are wide-angle PEs, and the EWAPEs are the extra-wide-angle PEs. The subscript "eff" indicates the effective sound-speed approximation. (The horizontal axis is truncated before $90^{\circ}$ because some of the solutions become infinite.)

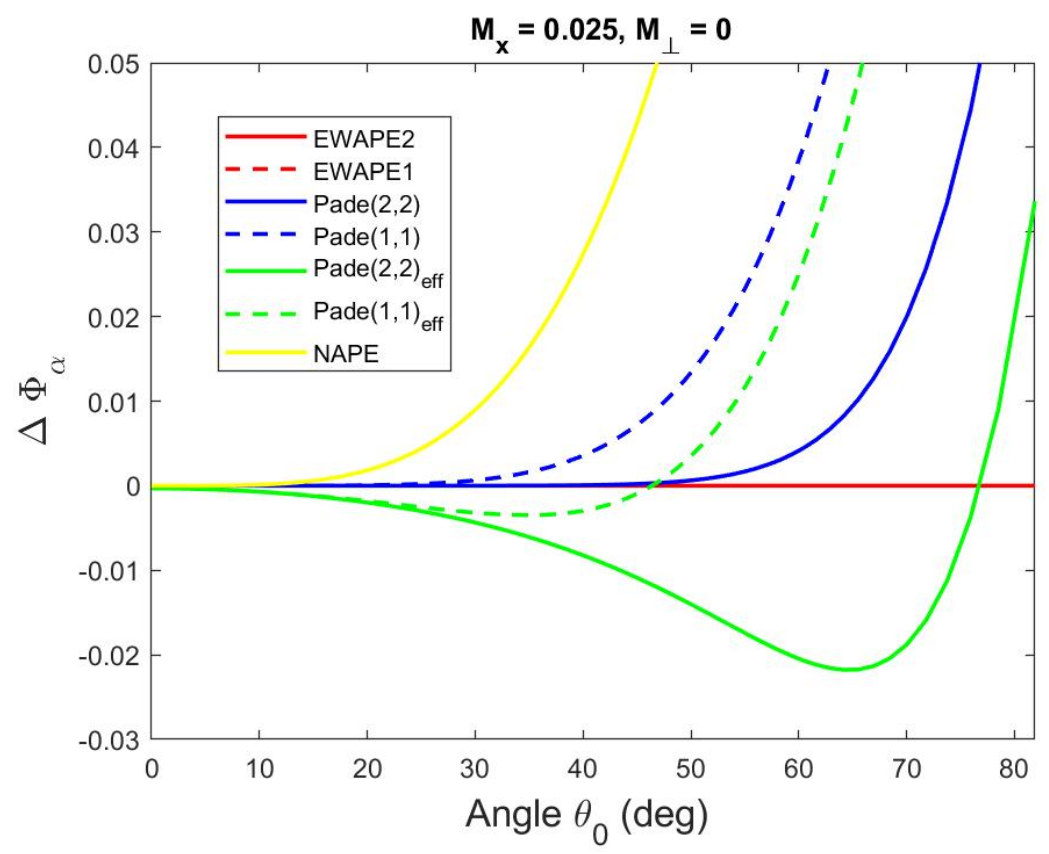

Figure 2. Same as Figure 1, except that for this calculation, the Mach number parallel to the propagation is $\boldsymbol{M}_{\boldsymbol{x}}=\mathbf{0}$, whereas the Mach number perpendicular to the propagation is $M_{\perp}=\mathbf{0 . 0 2 5}$.

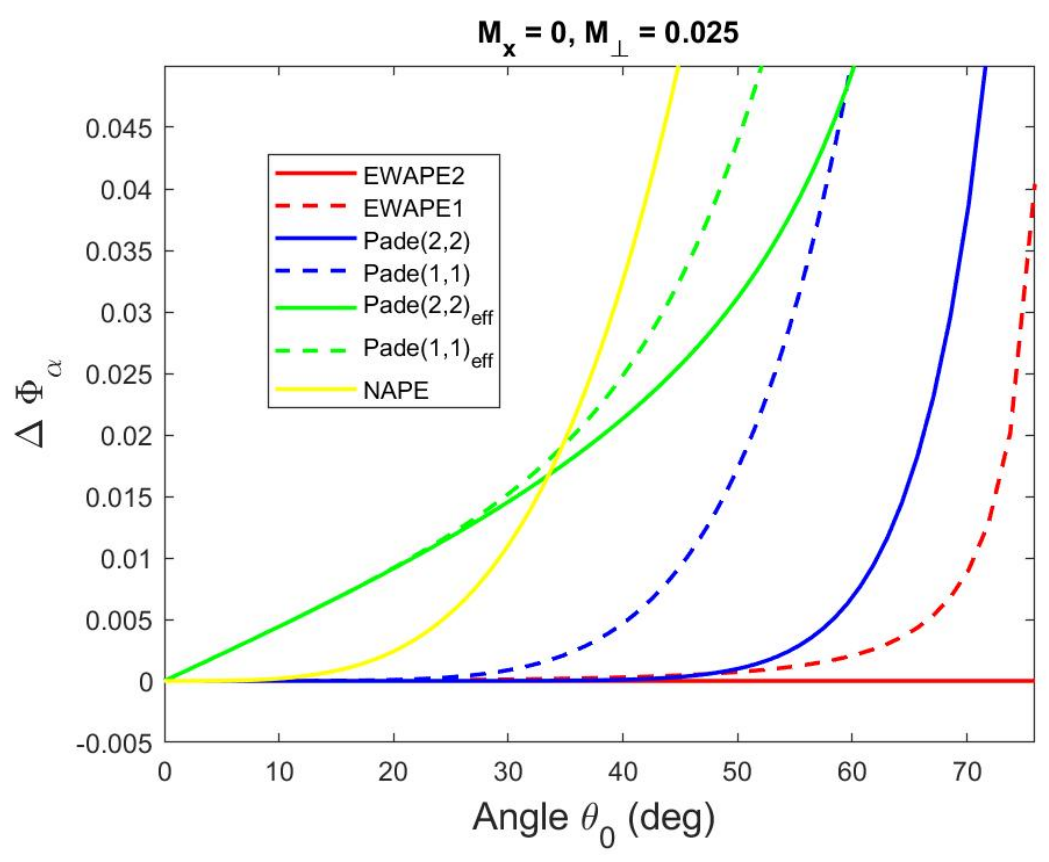


Figure 3 compares spectral calculations by three approaches, namely the basic NAPE (labelled simply "PE" in the figure title), a wide-angle Green's function PE (extended version of the original GFPE from Gilbert and Di [1993]), and the EWAPE. The NAPE calculation is narrow angle with respect to both the propagation angle and scattering by turbulence. The GFPE calculation is wide with respect to the propagation but narrow with respect to the scattering. The EWAPE is wide with respect to both. The atmosphere for all three cases is identical, incorporating a von Karman model of turbulence with moderately windy and sunny conditions. The source is a $500 \mathrm{~Hz}$ Gaussian piston source located at 40 meters (m) above the ground. The propagation was calculated with 40 instances of the atmosphere, after which the results were ensemble averaged. The narrowangle scattering of the GFPE is evident by the fact that close to on-axis, the GFPE and the NAPE are very similar. The EWAPE, on the other hand, exhibits noticeably different behavior in the same region of propagation due to inclusion of wide-angle scattering.

Figure 3. Comparison of basic narrow-angle PE (left), Green's function PE (center), and EWAPE (right) for propagation in a turbulent atmosphere.
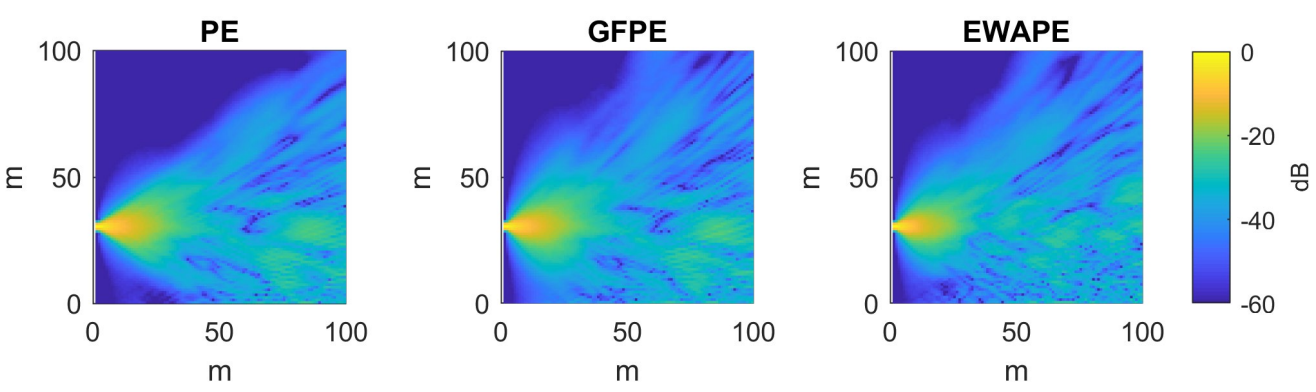

\subsection{Approximation for short wavelengths/large inhomogeneities}

The EWAPE derivation starts with the basic wave equation for a moving medium, namely Eq. (2.88) in the book by Ostashev and Wilson (2015). The derivatives of the wind velocity and density appearing in this equation can be neglected at relatively high frequencies, which results in

$$
\left[\frac{\partial^{2}}{\partial x^{2}}+\frac{\partial^{2}}{\partial \mathbf{r}^{2}}+k_{0}^{2}\left(1+\varepsilon+\frac{2 i}{k_{0} c_{0}} \mathbf{v} \cdot \nabla\right)\right] p(x, \mathbf{r})=0 .
$$

Here, $p(x, \mathbf{r})$ is the complex sound pressure, $x$ is the nominal propagation direction, and $\mathbf{r}=(y, z)$ are the coordinates transverse to this direction. 
Furthermore, $\varepsilon=c_{0}^{2} / c^{2}-1, c$ is the sound speed, $c_{0}$ is the reference sound speed, $k_{0}=2 \pi f / c_{0}$ is the reference wavenumber, $f$ is the frequency, and $\mathbf{v}$ is the wind velocity.

Although it may initially seem strange to make a high-frequency approximation for a project involving infrasound, bear in mind that what matters in the present context is the size of the acoustic wavelength relative to the size of the refractive inhomogeneities of interest. The wavelength at $10 \mathrm{~Hz}$ is approximately $33 \mathrm{~m}$. This value is short in comparison to the thickness of vertical refractive layers in the troposphere and stratosphere (e.g., the vertical extent of the jet stream), which are typically hundreds or thousands of meters. Thus, the approximation is quite reasonable in the present context. It is also consistent with assumptions underlying previous derivations of parabolic equations for non-moving media, which have been previously applied to infrasound propagation in the atmosphere.

Introducing now the longitudinal and transverse Mach numbers, $M_{x}=$ $v_{x} / c_{0}$ and $\mathbf{M}_{\perp}=\mathbf{v}_{\perp} / c_{0}$ (where $\mathbf{v}_{\perp}=\left(v_{y}, v_{z}\right)$ is the transverse wind), the previous result can be written

$$
\left[\frac{\partial^{2}}{\partial x^{2}}+2 i k_{0} M_{x} \frac{\partial}{\partial x}+k_{0}^{2}(1+\hat{\mu}+\hat{\eta})\right] p(x, \mathbf{r})=0,
$$

where we have introduced the operators

$$
\hat{\mu}=\frac{1}{k_{0}^{2}} \frac{\partial^{2}}{\partial \mathbf{r}^{2}} \text { and } \hat{\eta}=\varepsilon+\frac{2 i}{k_{0}} \mathbf{M}_{\perp} \cdot \frac{\partial}{\partial \mathbf{r}} .
$$

Factoring into waves traveling in the $+x$ and $-x$ directions, and retaining only the former, we arrive at the following one-way, pseudo-differential equation:

$$
\left[\frac{\partial}{\partial x}+i k_{0} M_{x}-i k_{0} \sqrt{1+\hat{\mu}+\hat{\eta}}\right] p(x, \mathbf{r})=0 .
$$

For a motionless medium $(\mathbf{v}=0)$, this result coincides with EWAPE2 in Ostashev et al. (2019). 


\subsection{Padé series solution}

Padé series are used to approximate a given function using a series of rational functions. They provide the most accurate approximation, to a given order, of the function. This contrasts with Taylor series, which are non-rational power series and provide less accuracy. Padé approximations have become widely used for parabolic equation solutions.

Let us approximate the pseudo-differential operator $\hat{Q}=\sqrt{1+\hat{\mu}+\hat{\eta}}$ in Eq. (4) with a Padé $(n, n)$ series as described in Collins (1993), namely

$$
\hat{Q}=1+\sum_{j=1}^{n} \frac{a_{j, n}(\hat{\mu}+\hat{\eta})}{1+b_{j, n}(\hat{\mu}+\hat{\eta})},
$$

where the $a_{j, n}$ and $b_{j, n}$ are numerical coefficients. Substituting the Padé series, Eq. (5), and setting $p(x, \mathbf{r})=e^{i k_{0} x} \hat{p}(x, \mathbf{r})$, Eq. (4) becomes

$$
\left[\frac{\partial}{\partial x}+i k_{0} M_{x}-i k_{0} \sum_{j=1}^{n} \frac{a_{j, n}(\hat{\mu}+\hat{\eta})}{1+b_{j, n}(\hat{\mu}+\hat{\eta})}\right] \hat{p}(x, \mathbf{r})=0 .
$$

In the following, we will assume that there is no transverse wind and that the derivatives in the $y$-direction can be neglected. (The latter approximation is typically made for PE calculations in the far field. See, for example, West et al. [1992].) The operators, Eq. (3), then simplify to

$$
\hat{\mu}=\frac{1}{k_{0}^{2}} \frac{\partial^{2}}{\partial z^{2}} \text { and } \hat{\eta}=\varepsilon .
$$

As we shall see in Section 4.1, the system specified by Eqs. (6) and (7) is relatively simple to solve as a straightforward generalization of existing parabolic equation solution methods. 


\section{Computational Methods}

This section describes the computational methods that were employed for the sound propagation and weather and how these were interfaced together.

\subsection{Numerical solution of the parabolic equation}

In this subsection, we discuss the numerical solution of Padé approximations to the WAPE, which were described in Section 3.4.

\subsubsection{Padé $(1,1)$ approximation}

Let us first consider the relatively simple Padé $(1,1)$ approximation, for which $a_{1,1}=1 / 2$ and $b_{1,1}=1 / 4$. (See, for example, Collins [1993].) Eq. (6) then becomes

$$
\begin{aligned}
{\left[\left(\frac{\partial}{\partial x}+i k_{0} M_{x}\right)\right.} & \left(1+b_{1,1}\left(\varepsilon+\frac{1}{k_{0}^{2}} \frac{\partial^{2}}{\partial z^{2}}\right)\right) \\
& \left.-i k_{0} a_{1,1}\left(\varepsilon+\frac{1}{k_{0}^{2}} \frac{\partial^{2}}{\partial z^{2}}\right)\right] \hat{p}(x, z)=0 .
\end{aligned}
$$

The numerical solution as presented in Ostashev and Wilson (2015) is based on casting Eq. (8) in the following form (Eq. [11.78] in Ostashev and Wilson [2015]):

$$
\Psi_{1}(x, z) \frac{\partial \hat{p}}{\partial x}=i k_{0} \Psi_{2}(x, z) \hat{p}(x, z),
$$

where $\Psi_{1}(x, z)$ and $\Psi_{2}(x, z)$ are operators with the general form

$$
\Psi_{m}(x, z)=\sum_{n} h_{m, n}\left(\frac{1}{k_{0}} \frac{\partial}{\partial z}\right)^{n} .
$$

Comparing Eqs. (8) and (9), we find the following non-zero values for the $h_{m, n}$ :

$$
\begin{array}{r}
h_{1,0}=1+b_{1,1} \varepsilon, \\
h_{1,2}=b_{1,1},
\end{array}
$$




$$
h_{2,0}=a_{1,1} \varepsilon-\left(1+b_{1,1} \varepsilon\right) M_{x}
$$

and

$$
h_{2,2}=a_{1,1}-b_{1,1} M_{x} .
$$

With these values for the coefficients, numerical solution by the CrankNicholson method proceeds in the manner described in Section 11.2.2 of Ostashev and Wilson (2015). As with the narrow-angle case (which corresponds to the preceding equations with $a_{1,1}=1 / 2$ and $b_{1,1}=1 / 4$ ), the Padé $(1,1)$ formulation here involves derivatives in $z$ up to second order, and thus entails solution of a tridiagonal matrix equation. Thus, the formulation here involves only minimal modifications to existing CrankNicholson NAPE codes and is just as computationally efficient.

The coefficients in Eq. (10) must furthermore be modified at the lower and upper boundaries to account for ground impedance and a radiation condition, respectively. The reader is referred to West et al. (1992), Salomons (2002), and Ostashev and Wilson (2015) for details. For WAPE calculations, a wide-angle starter should also be used. For this purpose, we employ the wide-angle starter derived by Salomons (2002).

\subsubsection{Padé $(2,2)$ approximation}

We next consider the Padé $(2,2)$ approximation, for which $a_{1,2}, a_{2,2}, b_{1,2}$, and $b_{2,2}$ are non-zero. (We will discuss values for these coefficients at the end of the section.) Eq. (6) becomes, after some algebra,

$$
\begin{gathered}
{\left[1+b_{1,2}\left(\varepsilon+\frac{1}{k_{0}^{2}} \frac{\partial^{2}}{\partial z^{2}}\right)\right]\left[1+b_{2,2}\left(\varepsilon+\frac{1}{k_{0}^{2}} \frac{\partial^{2}}{\partial z^{2}}\right)\right] \frac{\partial \hat{p}(x, z)}{\partial x}} \\
=i k_{0}\left\{-M_{x}\left[1+b_{1,2}\left(\varepsilon+\frac{1}{k_{0}^{2}} \frac{\partial^{2}}{\partial z^{2}}\right)\right]\left[1+b_{2,2}\left(\varepsilon+\frac{1}{k_{0}^{2}} \frac{\partial^{2}}{\partial z^{2}}\right)\right]\right. \\
+a_{1,2}\left(\varepsilon+\frac{1}{k_{0}^{2}} \frac{\partial^{2}}{\partial z^{2}}\right)\left[1+b_{2,2}\left(\varepsilon+\frac{1}{k_{0}^{2}} \frac{\partial^{2}}{\partial z^{2}}\right)\right] \\
\left.+a_{2,2}\left(\varepsilon+\frac{1}{k_{0}^{2}} \frac{\partial^{2}}{\partial z^{2}}\right)\left[1+b_{1,2}\left(\varepsilon+\frac{1}{k_{0}^{2}} \frac{\partial^{2}}{\partial z^{2}}\right)\right]\right\} \hat{p}(x, z) .
\end{gathered}
$$

Comparing Eqs. (11) and (9), we find the following non-zero values for the $h_{m, n}$ : 


$$
\begin{gathered}
h_{1,0}=\left(1+b_{1,2} \varepsilon\right)\left(1+b_{2,2} \varepsilon\right), \\
h_{1,2}=b_{1,2}\left(1+b_{2,2} \varepsilon\right)+b_{2,2}\left(1+b_{1,2} \varepsilon\right), \\
h_{1,4}=b_{1,2} b_{2,2}, \\
h_{2,0}=-M_{x}\left(1+b_{1,2} \varepsilon\right)\left(1+b_{2,2} \varepsilon\right)+a_{1,2} \varepsilon\left(1+b_{2,2} \varepsilon\right) \\
+a_{2,2} \varepsilon\left(1+b_{1,2} \varepsilon\right), \\
h_{2,2}=-M_{x} b_{1,2}\left(1+b_{2,2} \varepsilon\right)-M_{x} b_{2,2}\left(1+b_{1,2} \varepsilon\right)+a_{1,2}\left(1+2 b_{2,2} \varepsilon\right) \\
+a_{2,2}\left(1+2 b_{1,2} \varepsilon\right),
\end{gathered}
$$

and

$$
h_{2,4}=\left(-M_{x} b_{1,2}+a_{1,2}\right) b_{2,2}+a_{2,2} b_{1,2} \text {. }
$$

According to Yevick and Thomson (2000), the following values are standard for WAPEs, as based on matching terms in the Padé approximation to the square root operator:

$$
\begin{gathered}
a_{j, n}=\frac{2}{2 n+1} \sin ^{2} \frac{j \pi}{2 n+1}, \\
b_{j, n}=\sin ^{2} \frac{j \pi}{2 n+1} .
\end{gathered}
$$

These formulas lead to $a_{1,2}=0.138196601125 \ldots, a_{2,2}=$ $0.361803398875 \ldots, b_{1,2}=0.361803398875 \ldots$, and $b_{2,2}=$ $0.095491502813 \ldots$ With these values, we can calculate the $h_{m, n}$ coefficients.

In addition to deriving values for the $h_{m, n}$ coefficients, it is also necessary to implement the finite differencing scheme. Since the equations for the Padé $(2,2)$ WAPE involve derivatives up to fourth order, the finite differencing scheme becomes quite a bit more complex in comparison to the Padé $(1,1)$ WAPE, which involves derivatives up to only second order. To determine the correct finite difference coefficients with second-order accuracy, the iterative method of Fornberg (1988) was implemented. This leads to a pentadiagonal matrix equation for the Padé $(2,2)$ WAPE, as opposed to the familiar tridiagonal matrix equation from the narrow-angle or Padé $(1,1)$ WAPE. The pentadiagonal system can be solved directly and efficiently with Matlab's sparse matrix operations. A similar formulation 
with a pentadiagonal system was considered previously for WAPEs in ocean acoustics by Saad and Lee (1986). Those authors, however, did not include motion in the propagation medium.

Interestingly, the calculations by all three methods (NAPE, Padé $(1,1)$ WAPE, and Padé $(2,2)$ WAPE) were found to have essentially the same calculation time for some low-frequency example problems. It came as a surprise that the Matlab sparse matrix library solved the pentadiagonal system in essentially the same amount of time as it did the tridiagonal system. This behavior may not hold for larger computational grids (i.e., higher frequencies).

Although the Padé $(2,2)$ WAPE implementation was successful from the standpoint of calculation time, it was not from the standpoint of stability. Despite taking great care in deriving the coefficients and implementing the calculation, the calculated fields would grow infinite after several dozen range steps. After experimenting extensively with various formulations for starters and boundary conditions, it became apparent that the fundamental issue is the values of the coefficients in the Padé $(2,2)$ approximation (the values for $a_{1,2}, a_{2,2}, b_{1,2}$, and $b_{2,2}$ ). As described in papers by Collins $(1991,1993)$, these coefficients need to be deliberately chosen in a manner that creates a stable solution. While the standard coefficients for the Padé $(1,1)$ WAPE lead to a stable solution, this is apparently not the case for the Padé $(2,2)$ WAPE. Collins (1991) discusses how stable versions of these coefficients, which are complex rather than real, can be derived and provides his suggested values in Table I of the 1991 paper. However, those values were specifically derived for elastic wave propagation in the ocean, rather than for propagation above an impedance ground boundary condition in the atmosphere.

As a test, we implemented calculations with the values in Table I of Collins (1991). The calculation did indeed stabilize. However, the results were very inaccurate. Collins (1991) indicates that "[t]his Padé series is slightly less accurate than the Padé series of (Eqs. [13] and [14]) because one less derivative is required to match. This trade-off of accuracy for stability is an excellent bargain." But, for the classes of problems of interest in the present study, the utility of this "bargain" is less evident. Since the Padé $(1,1)$ WAPE is accurate and stable, the Padé $(2,2)$ WAPE does not appear to be a satisfactory approach if we have to sacrifice accuracy substantially to 
achieve stability, in order to have a solution that is valid at a somewhat larger angle.

The situation could possibly be remedied by deriving new Padé coefficients by some other method. In Collins (1991), below Eq. (12), there is a paragraph which suggests a procedure for stabilizing the Padé coefficients. The procedure involves setting a small parameter $\epsilon_{1}$ to a non-zero value. (When the parameter equals zero, Eqs. (9) and (10) are recovered.) However, Collins does not actually provide coefficient values derived in this manner. Perhaps by setting $\epsilon_{1}$ to a very small non-zero value, the coefficients may produce a stable result without significant attenuation and loss of accuracy. While this could be a worthwhile topic for future research, we considered such a derivation beyond the scope of the present project.

We also mention that, in addition to the direct implementation of the fourth-order derivative as described here, the Padé $(2,2)$ WAPE can be implemented using the split-step method described in Collins (1993). That approach was also tried and suffered from the same stability issues related to the Padé coefficients.

\subsection{Polar Weather Research and Forecasting (PWRF)}

For the purposes of this project, we wish to model many diverse Arctic phenomena occurring across a range of spatial scales, from major stratospheric warmings to small scale inversions and katabatic wind features. Of course, trade-offs are inevitable when endeavoring to model such a diverse range of phenomena. It was decided that the most appropriate, readily available dataset is the ASRv2 (Bromwich et al. 2018), produced by the Ohio State University Byrd Polar Research Institute and provided through NCAR's Computational and Information Systems Lab (CISL) (NCAR/UCAR/OSU 2017). The reanalysis blends observational data with the PWRF weather model (using the Advanced Research WRF [ARW] solver) (Hines et al. 2008; Bromwich et al. 2018) through 4-D atmospheric variational data assimilation and land data assimilation. The data assimilation is an important advantage of this dataset, as it improves the realism. PWRF includes sea-ice surface, ice-cloud microphysics, and other modifications to the standard WRF release (Wilson et al. 2011; Hines et al. 2015). It employs a rotated grid, for which the numerical pole lies outside the polar domain, to improve the numerical grid convergence. 
The ASRv2 dataset provides $15 \mathrm{~km}$ horizontal resolution over a pan-Arctic domain for the years 2000-2012. The data are stored on a staggered Arakawa C grid with $721 \times 721$ grid points, with a polar stereographic projection. In the vertical direction, this PWRF-based configuration uses a terrain-following dry hydrostatic pressure coordinate system with 71 model levels and a constant pressure surface at the model top of 10 millibars (mb). A numerical upper boundary condition helps to mitigate spurious reflections that adversely impact lower atmospheric model results. The terrain-following nature of the grid helps to ensure more reasonable representation of some phenomena of interest such as katabatic winds.

The $10 \mathrm{mb}$ model top corresponds to an altitude of about $26 \mathrm{~km}$, which is suitable for infrasound propagation calculations into the midstratosphere. This upper height limit is suitable for infrasound calculations out to ranges of several hundred kilometers and are of primary interest in Army applications. For longer-range propagation calculations, interactions with the mesosphere and thermosphere can be important, in which case the $26 \mathrm{~km}$ limitation becomes problematic. Therefore, to support such calculations, Gibson and Drob (2005) merged numerical weather predictions with additional datasets.

The ASRv2 data were subsampled in Network Common Data Form version 4 (netCDF4) format with respect to relevant quantities (wind, pressure, temperature, and humidity) for ingest and coordinate transformations within the EASEE software framework, as described in the next subsection.

\subsection{Interfacing PWRF to the parabolic equation}

To interface the PWRF output to sound propagation calculations, we leveraged the ERDC EASEE software. EASEE provides a well-developed framework capable of resampling environmental data specified in any geographic coordinate projection, such as the polar stereographic representation employed by PWRF, and an interface to propagation calculations performed in cylindrical coordinates, such as the parabolic equation.

EASEE includes a self-contained representation, or data abstraction layer, of the environment. The environment includes terrain surface (elevations and land cover), sub-surface (soil and seismic profiles), and 
atmospheric properties. The abstraction layer is one of the key features of the architecture, as it isolates the modeling capabilities integrated into EASEE from the particular data resources used to specify the environment. Whenever a new type of environmental data resource (e.g., terrain data or a weather forecast model) is interfaced with EASEE, it will become usable by all existing modeling capabilities. Conversely, whenever a new modeling capability is integrated with EASEE, it can be used with all types of environmental data recognized by the abstraction layer. The process through which the environmental data abstraction layer is initialized and feeds into the propagation calculations is shown in Figure 4 .

Figure 4. The EASEE environmental abstraction layer (EnvironScenario) is constructed from a variety of data resources for terrain elevation, land cover, soils, and atmospheric forecasts and models. The environmental data are converted to the propagator media, which are then used for signal propagation modeling.

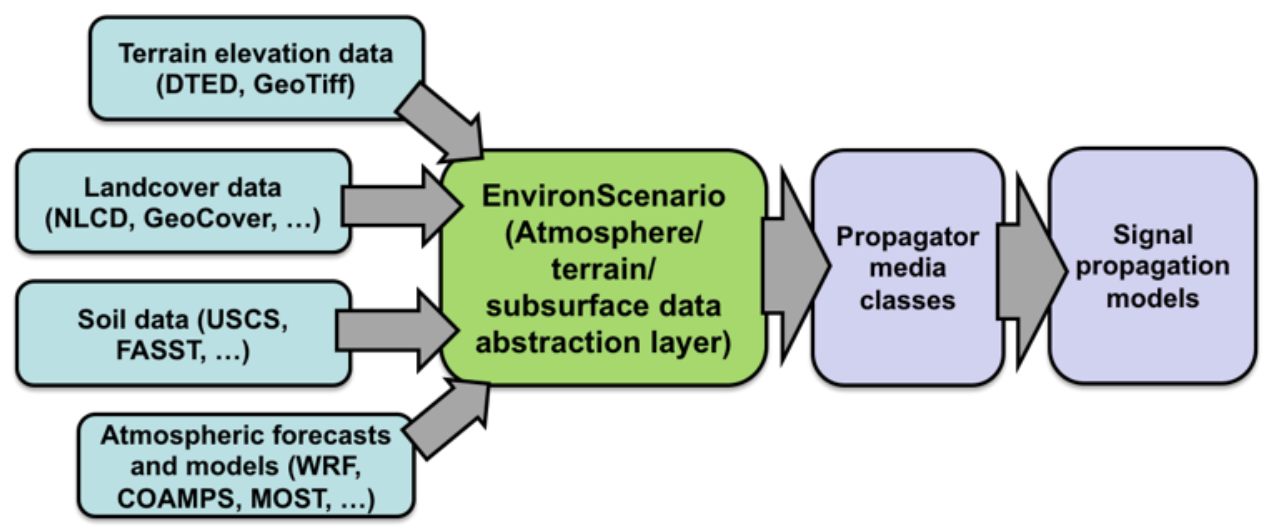

With the completion of EASEE v3 (Wilson et al. 2020), the environmental data abstraction layer was expanded to include full 3-D environmental variability. This includes loading of numerical weather forecast model data in 3-D. Prior to this project, EASEE could already initialize its atmospheric representation using several forecast models, namely (1) WRF, which is the primary forecast model used by the U.S. weather research community and the U.S. Army, (2) Coupled Ocean/Atmosphere Mesoscale Prediction System (COAMPS), which is the U.S. Navy's primary forecast model, and (3) Global Air-Land Weather Exploitation Model (GALWEM), which is a version of the U.K. Meteorology Office forecast model that was modified to meet the needs of the U.S. Air Force, who recently adopted it as their official mesoscale meteorology model. GALWEM is also now being used for Army operational weather support. With COAMPS, WRF, and 
GALWEM, EASEE supports all the major weather forecast models used by DoD.

New for this project was the ability to load data from the polar version of WRF (PWRF). The normal WRF model executes on a Lambert coordinate projection. PWRF, however, executes on a polar stereographic projection. This transformation was thus added to EASEE.

The WRF forecasts are loaded by Java classes called WrfLoader (for 1-D modeling) and WrfLoader3D (for 3-D modeling). These classes load WRF data from files in the Network Common Data Form (NetCDF) format and process them into vertical profiles (for 1-D) or full 3-D grids. The loader handles conversions between the geopotential height (the vertical coordinate used by the forecast model) and the actual height above ground level as needed for the acoustical calculations. 


\section{Simulation Results}

This section discusses significant Arctic weather phenomena as found in the ASRv2 PWRF simulation dataset (Section 5.1), followed by example infrasound calculations showing the impact of these weather phenomena (Section 5.2). Lastly, infrasound propagation characteristics at temperate and Arctic latitudes, as based on measured atmospheric profiles, are compared (Section 5.3).

\subsection{Arctic weather}

The methodology employed for the PWRF simulations was described in Section 4.2. In this section, we provide some example results and discussion of interesting Arctic weather phenomena.

One such phenomenon is SSW events, which are "large and rapid temperature increases in the winter polar stratosphere ... associated with a complete reversal of the climatological westerly winds ..." (Butler et al. 2017). The connection of SSW events to the polar vortex, which can lead to prolonged cold-air outbreaks at temperate latitudes, has drawn much interest. Stratospheric temperature changes of tens of degrees Kelvin (K) can occur (Butler et al. 2017).

Major SSWs are effectively hemispheric phenomena resulting from lowlatitude perturbations that then propagate poleward. As a result of these interactions, the stratospheric polar jet (stream of higher wind speeds) experiences exponential wobble and Rossby wave breaking, which bifurcates the otherwise singular westerly winter jet and polar vortex into two. This ultimately results in a zonally averaged warming of the polar stratosphere through an expulsion of equatorward heat to space via the polar atmosphere, in a process akin to boundary-layer turbulent bursts. However, the winter equator-to-pole gradient of, for example, low-level, near-surface temperature eventually approaches a systemic equilibrium (reduction to more sustainable meridional gradients), resulting in significant variance divergence of the stratospheric polar vortex winds and temperatures, a new behavior regime, and subsequent recovery occurring over approximately one month. From the perspective of infrasound propagation, what might otherwise be expected of a winter atmosphere (e.g., zonally averaged westerly polar jet) is greatly affected on an almost 
hemispheric scale such that vertical and horizontal gradients of temperature and wind invert.

Coy et al. (2015) describe such an event from the winter of 2012-2013 that peaked (in terms of temperature and wind reversal) around January 6 , 2013. This peak is evident in Figure 5, which depicts the zonal mean temperature during the winter of 2012-2013 (averaged north of $65^{\circ} \mathrm{N}$ at $50 \mathrm{mb})$.

Figure 5. Data from the Climate Prediction Center (CPC) of the National Oceanic and Atmospheric Administration (NOAA) depicting major stratospheric warming during the winter of 2012-2013. Temperature at $50 \mathrm{mb}$ averaged from $65-90^{\circ} \mathrm{N}$, with minimum, maximum, and mean temperature for the period of record (1979-2008).

50-hPa Zonal Mean Temperature for 2012 \& 2013

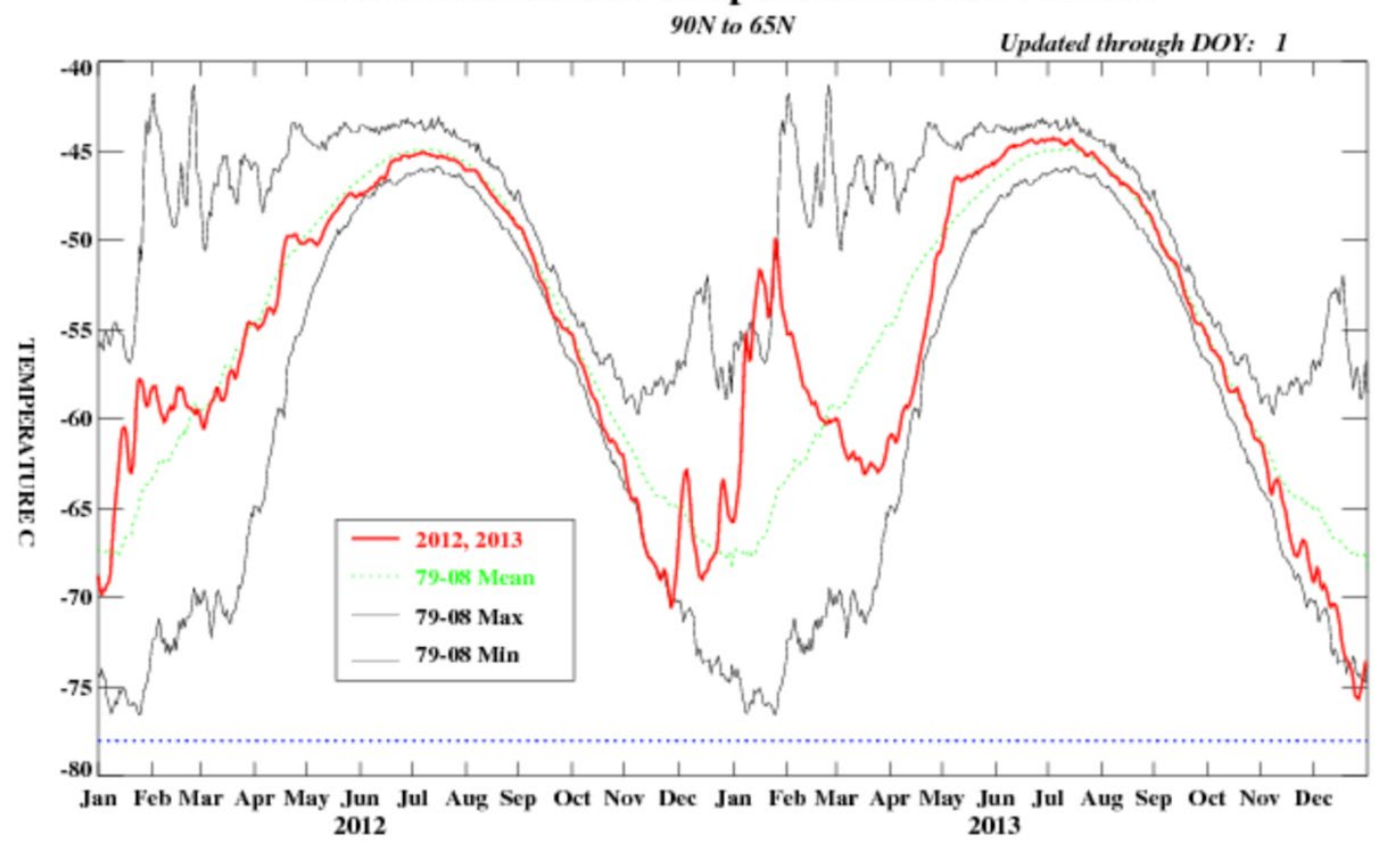

The pronounced bifurcation of the stratospheric polar vortex that is typical of these events is easily seen in the $50 \mathrm{mb}$ plot of geopotential height in Figure 6. (Here, $1 \mathrm{mb}=1$ hectopascal $(\mathrm{hPa})=100 \mathrm{~Pa}$.) The $50 \mathrm{mb}$ temperatures, as depicted in Figure 7, indicate a nearly $40 \mathrm{~K}$ increase over a broad region at that altitude relative to more typical temperatures for that date and time. 
Figure 6. Geopotential height (roughly the height of the $50 \mathrm{mb}$ pressure surface) for the peak of the major SSW event on 6 January 2013 highlighting the typical bifurcation of the stratospheric vortex with localized (and zonally averaged) reversal of the zonal wind (in the west-east direction).

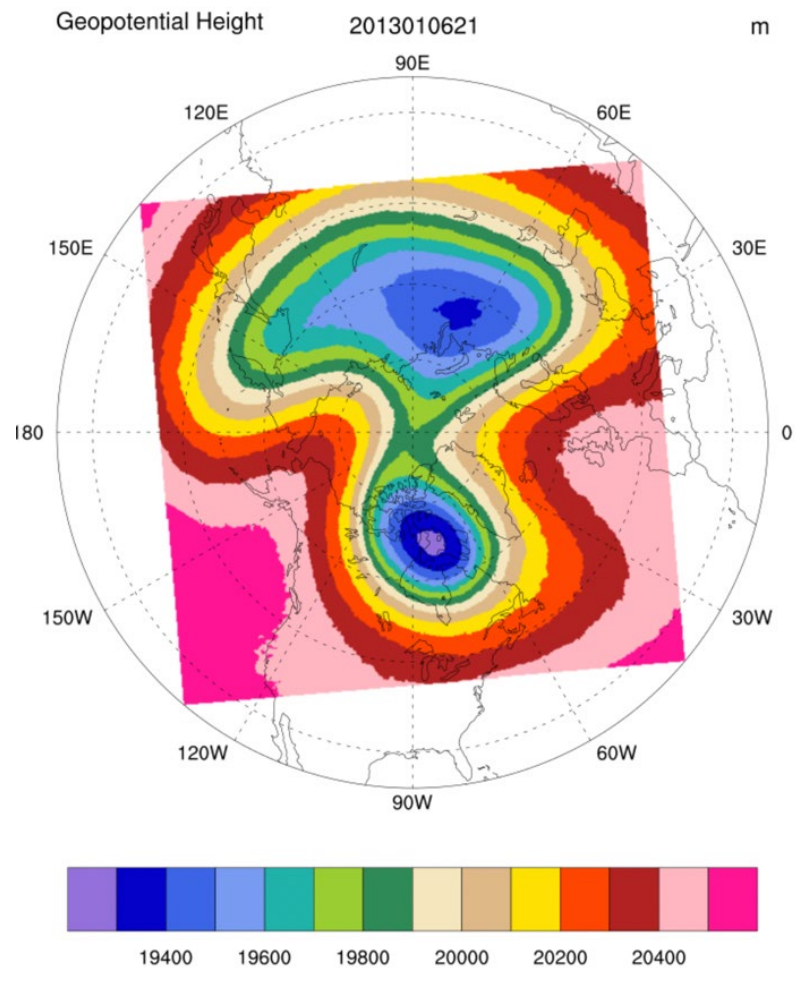

Let us consider a particular transect through the ASRv2 PWRF simulations at oZ (i.e., oooo Universal Coordinated Time, or UTC) on January 6, 2013, as shown in Figure 8. The profiles along this transect, which are shown in Figure 9, highlight the reversal of wind direction aloft that is typical of the major SSW event (e.g., dashed contours in the upper left of the figure). 
Figure $7.50 \mathrm{mb}$ surface temperatures on 6 January 2013 highlighting pronounced warm sectors that are tens of degrees Kelvin higher than normal for the date.

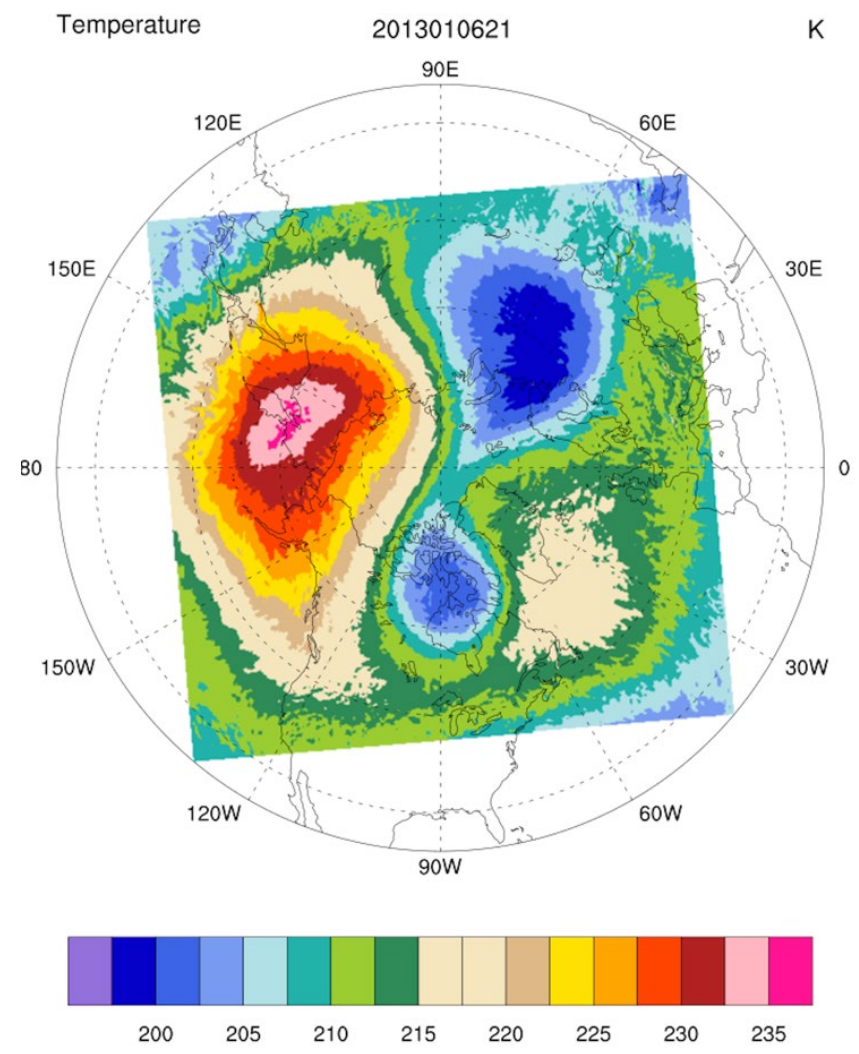

Figure 8. Blue transect geolocating the wind and temperature cross section in Figure 9.

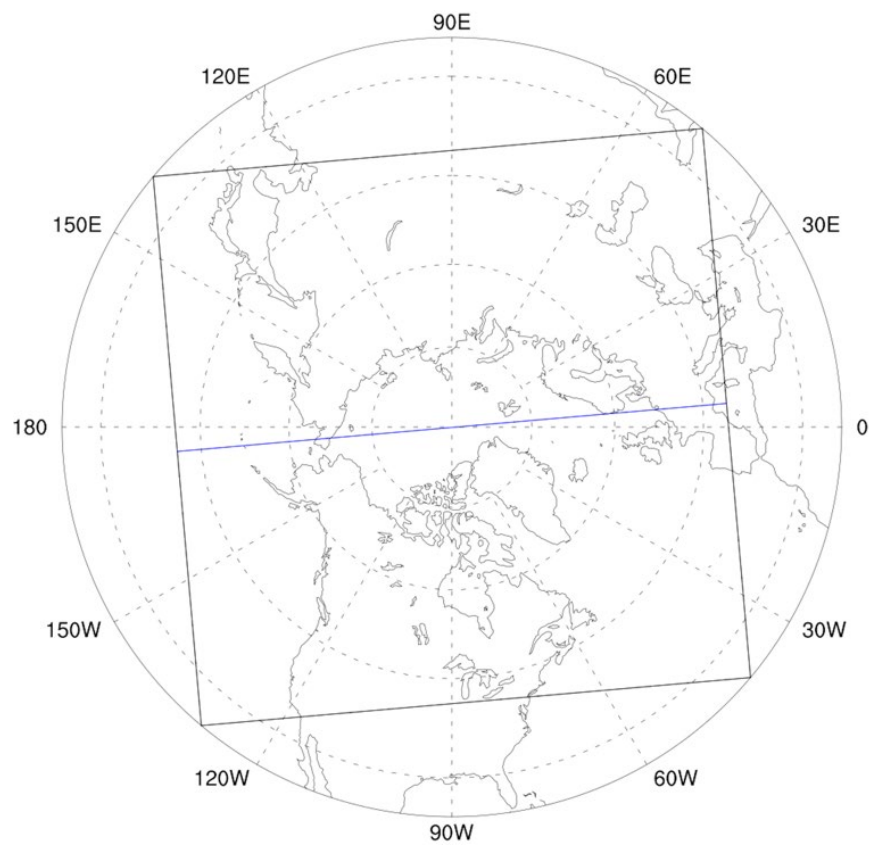


Figure 9. Zonal wind (solid and dashed black contours and labels) and air temperature at $\mathrm{OZ}$ on 6 January 2013 for latitude/longitude pairs along the x-axis of the figure. Note the warm perturbations and stratospheric jet reversal in the upper left of the figure.

Zonal Wind $(\mathrm{m} / \mathrm{s})$ and Temperature $(\mathrm{K})$

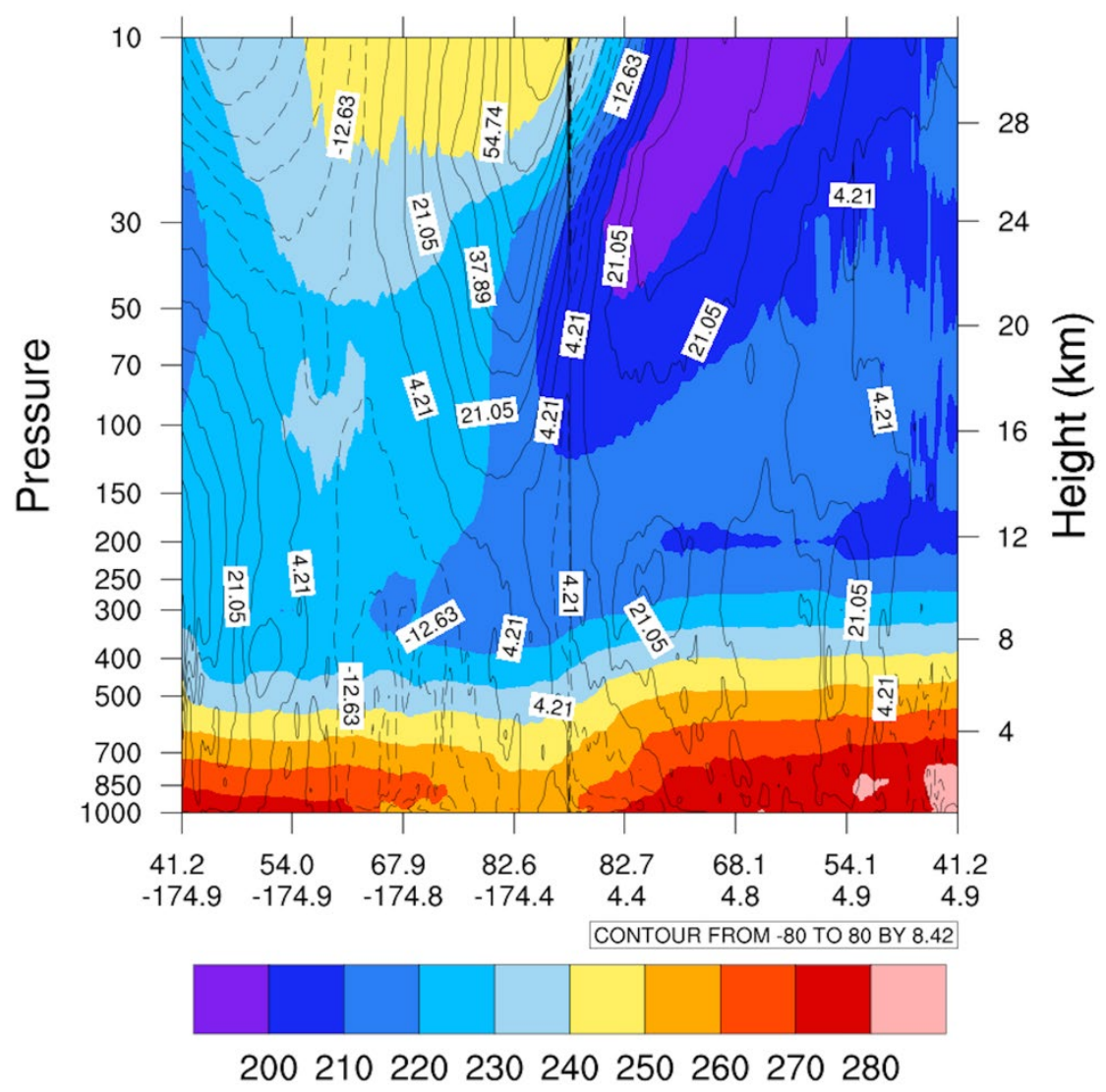

Another interesting feature at relatively large mesoscales is a polar low or "Arctic hurricane," defined as a small but intense cyclone that forms in cold polar air advected over warmer water. These vortices often form in the subpolar North Pacific or North Atlantic, equatorward of the sea ice margin. Horizontal scales range from several tens to several hundreds of kilometers. Regarding meteorological gradients that are important to acoustic propagation (e.g., refraction and ducting), temperature within these systems might be expected to decrease with height and with radial distance from the center of the storm, and wind speed might be expected to increase with height (with some locally higher wind speeds near the tropopause, approximately 5-10 km above the Earth's surface) and increase then decrease with radial distance from the center of the storm.

Some of these characteristics are especially notable in a historic example from early August 2012 known as "The Great Arctic Cyclone of 2012" due to its especially low surface pressure (minimum of $966 \mathrm{mb}$ ) and high 
sustained winds (approximately $36 \mathrm{~m} / \mathrm{s}$ ). This event is described more fully in Simmonds et al. (2012). The surface pressure map for this event, as depicted in the National Centers for Environmental Prediction Climate Forecast System Reanalysis, is shown in Figure 10. Such storms are known to interact with sea ice by inducing wave action on the ocean surface, which can break up the ice near its edges. These storms could significantly impact infrasound propagation through modification of both surface properties and the atmospheric profiles throughout the depth of the Arctic troposphere.

Figure 10. The "Great Arctic Cyclone of 2012" as seen in the surface pressure field at $21 Z$ on 6 August 2012 exhibiting an intense low-pressure core.

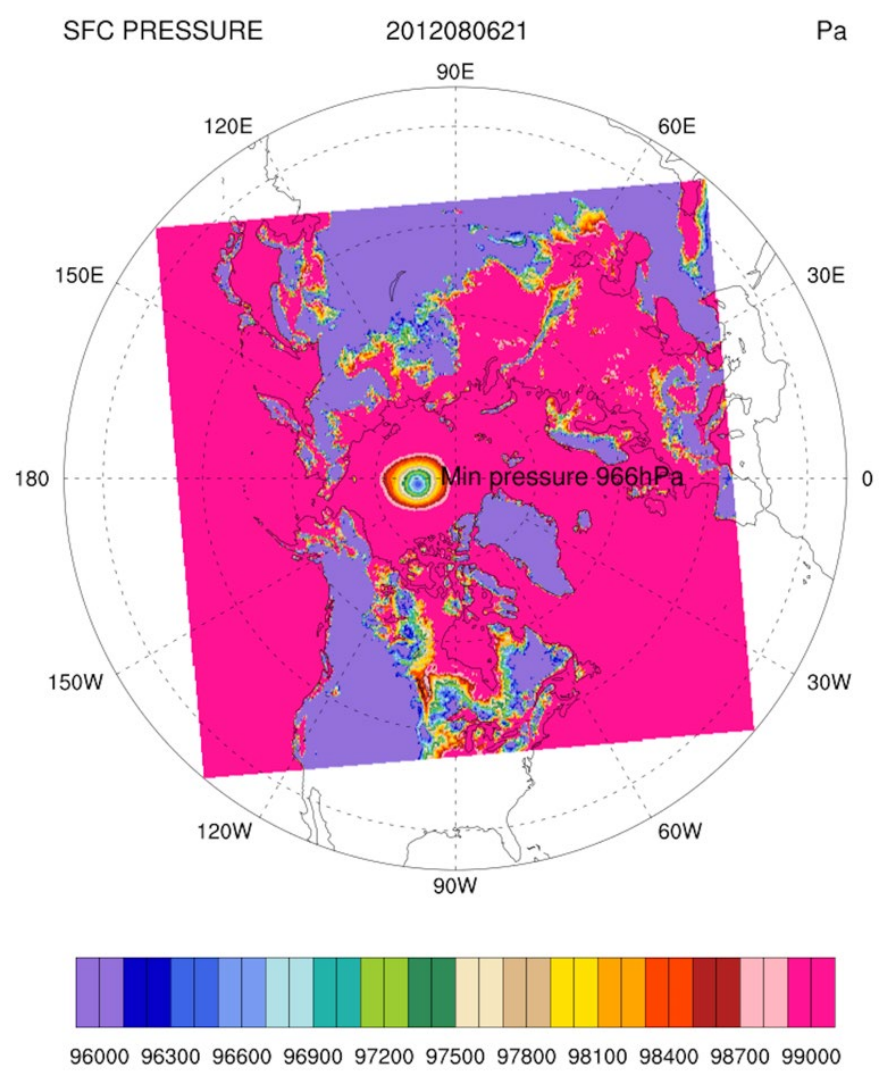

A vertical cross section of zonal wind through this system is depicted in Figure 11. The minimum in zonal wind speed near the center of the image is close to the low-pressure center or "eye" of the storm. 
Figure 11. A vertical cross section of the Great Arctic Cyclone of 2012 exhibiting strong jets (wind cores), temperature gradients, and a warm core or "eye." Color fill refers to zonal wind speed $(\mathrm{m} / \mathrm{s})$, and contour lines refer to air temperature $(\mathrm{K})$.

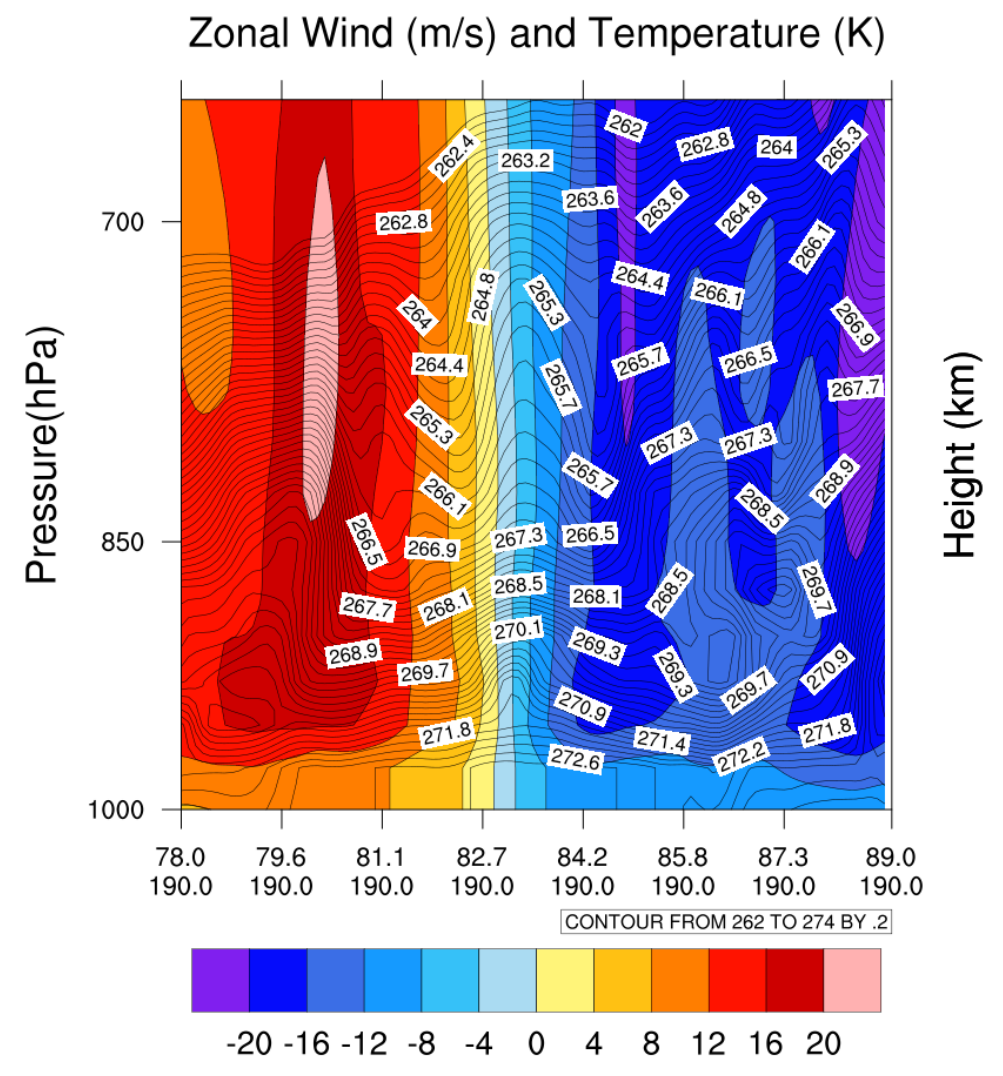

Longitude/Latitude

Additionally, various polar low events-including one peaking around 21 March 2001 (centered approximately at $66^{\circ} \mathrm{N}$ latitude and $10^{\circ} \mathrm{E}$ longitude) and one peaking around November 19, 2015 (centered approximately at $70^{\circ} \mathrm{N}$ latitude and $5^{\circ} \mathrm{E}$ longitude)-have occurred off the coast of Norway. A transect for the November event is depicted as a yellow line in Figure 12, and a vertical cross section for the event is shown in Figure 13. Note in Figure 13 the fairly typical temperature inversion with occlusion and overriding low-level jet (LLJ, lower left of the figure). 
Figure 12. A complex of polar lows off the coast of Norway at $12 Z$ on 19 November 2015 as depicted through the surface pressure field. The yellow line represents a transect through the core of one polar low that is highlighted in a vertical cross section in the following figure.

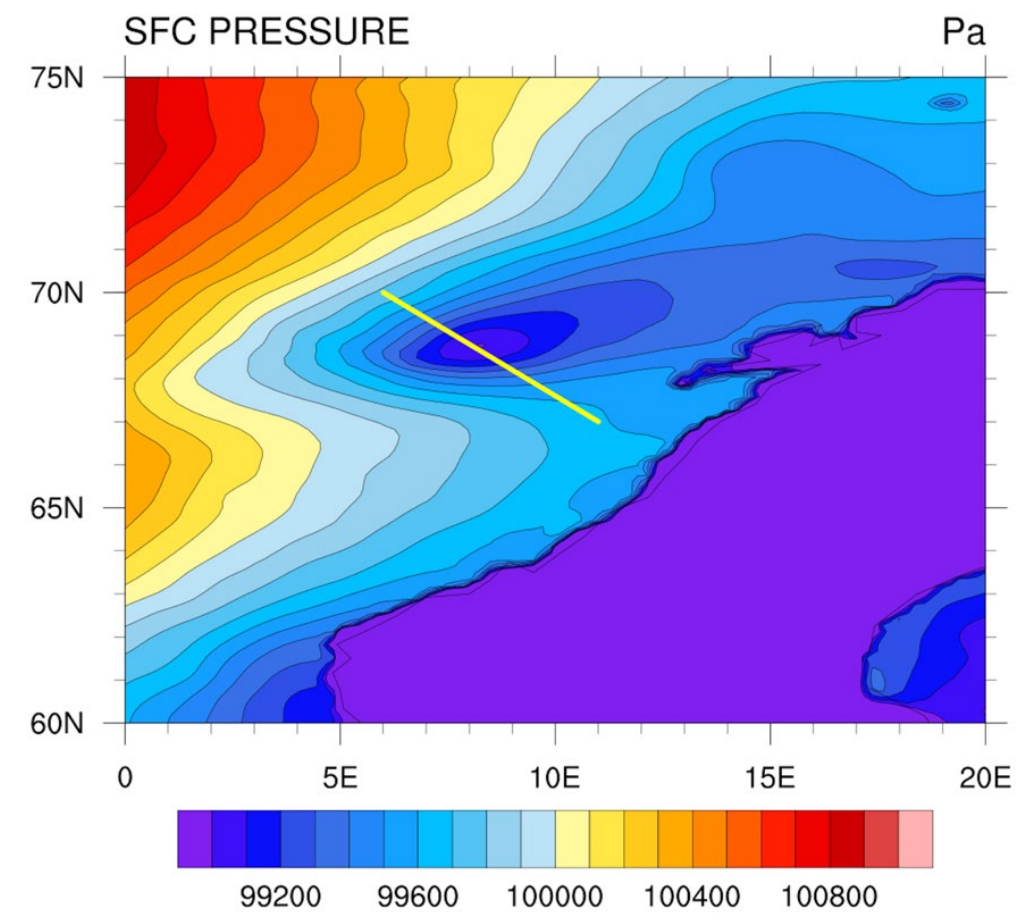


Figure 13. A vertical cross section of the polar low in Figure 12, showing temperature and zonal wind. Note the occlusion, temperature inversions, and a low-level jet (LU) in the lower left of the figure.

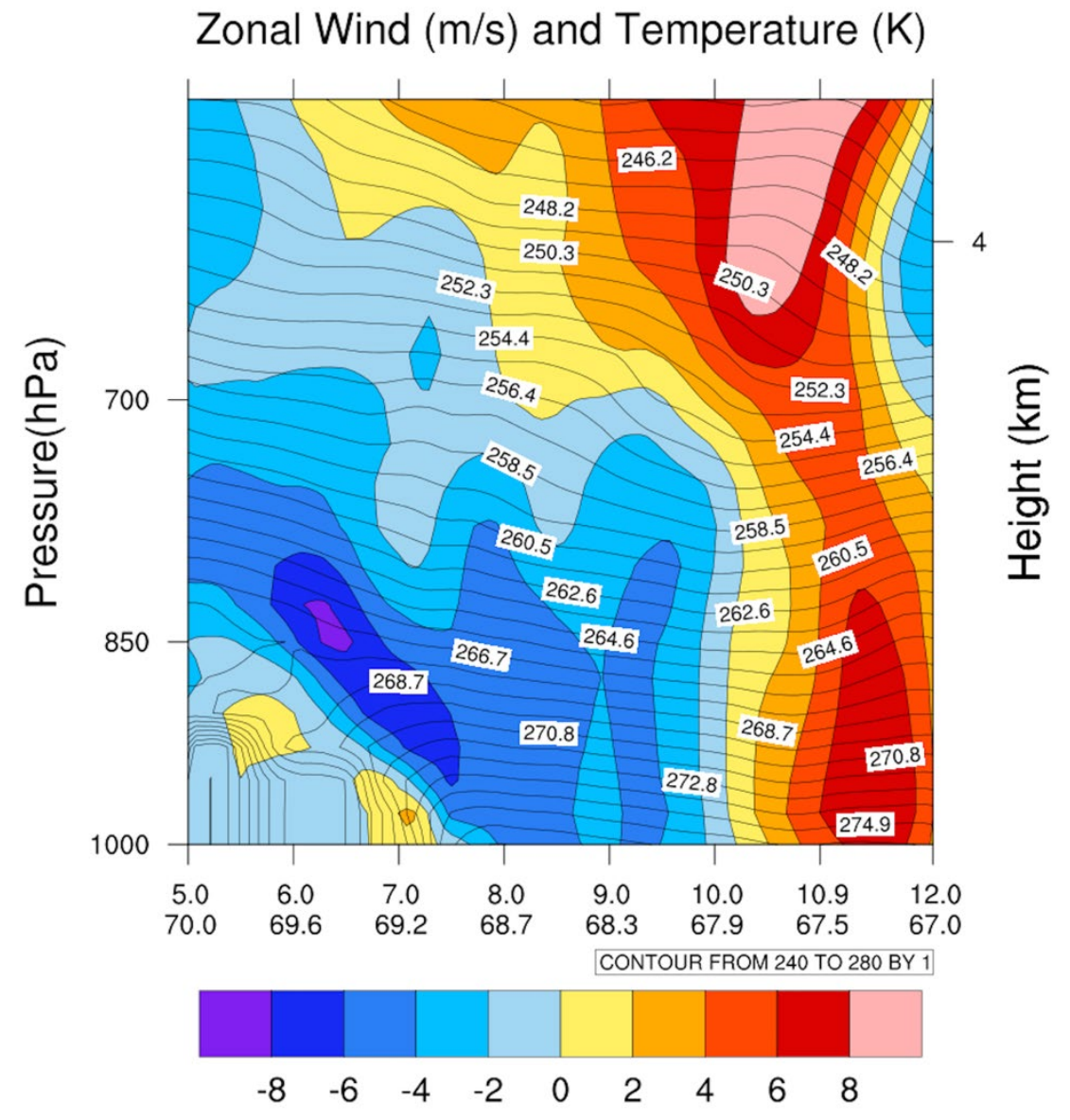

At smaller spatial scales (the meso-beta to micro) and lower altitudes, but still of interest for infrasound propagation, is the occurrence of LLJs in the Arctic. LLJs occur throughout the world and develop partly as a function of gradients of near-surface temperature, pressure, and humidity that, through baroclinicity, generate a solenoidal impulse to atmospheric motion in a "streak" or "sheet" of approximately geostrophic wind not far above Earth's surface (on the order of $1 \mathrm{~km}$ above ground level). Wind speeds in LLJs can be on the order of several tens of $\mathrm{m} / \mathrm{s}$, and the pronounced inversion of motion and temperature from what is otherwise typical above LLJs can affect acoustic refraction. LLJs have been observed and simulated to run along the baroclinicity encountered at sea ice edges, as was evident during the Sea State cruise in the fall of 2015 in the Chukchi/Beaufort Sea region (Guest et al. 2018). During this cruise, winds of $8 \mathrm{~m} / \mathrm{s}$ extended through a capping inversion to $2-3 \mathrm{~km}$ altitude, with winds decreasing toward the surface in the boundary layer due to friction. 
The width (crosswind dimensions) of the jets was $250-400 \mathrm{~km}$, with windward orientation roughly parallel with the sea ice edge. Jakobson et al. (2008) document LLJ characteristics more generally over the Arctic, with a jet noted and described on 10 August 2007 near the end of the mission (at about $86^{\circ} \mathrm{N}$ and $150^{\circ} \mathrm{W}$ ) off the drifting ice station Tara, not far from the ice margin, as seen in Figure 14 (along the transect delineated in red).

Figure 14. Red transect near the sea ice edge to geolocate the LU via cross section on August 10, 2007.

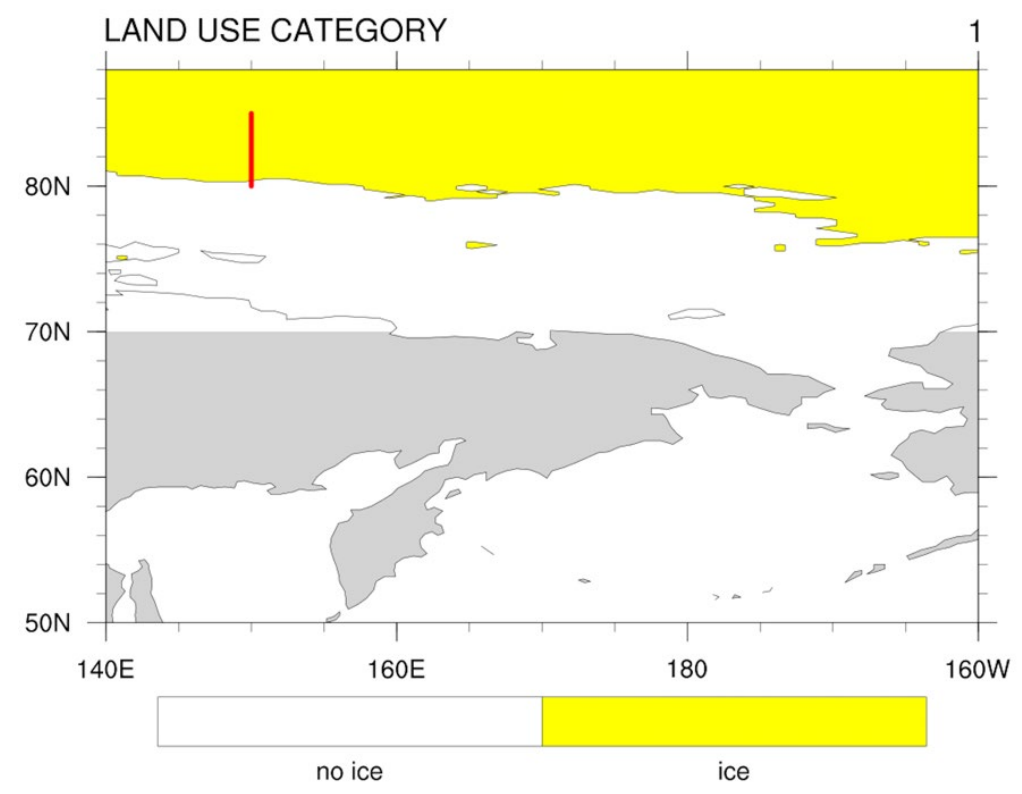

Figure 15 and in Figure 16, where relative extrema for wind and temperature are evident above and below the jet core (see Jakobson et al. 2008 for more details). The panel in Figure 15 is a cross section through the jets and relative minima and maxima of wind blowing in significant part parallel to the ice edge and temperature and density, the isoclines of which being oriented parallel to the ice edge. The jets are generally into/out of the panel. Baroclinicity (density gradients in vertical and horizontal) sets up parallel to the ice edge, resulting in a jet parallel to the ice edge (i.e., density/pressure gradient and Coriolis act together to steer flow/wind parallel to ice edge). 
Figure 15. Several jets (the blue, finger-like protrusions of high wind speed) in a vertical cross section near the sea ice edge, with a characteristic temperature inversion over and near the near-surface jet core.

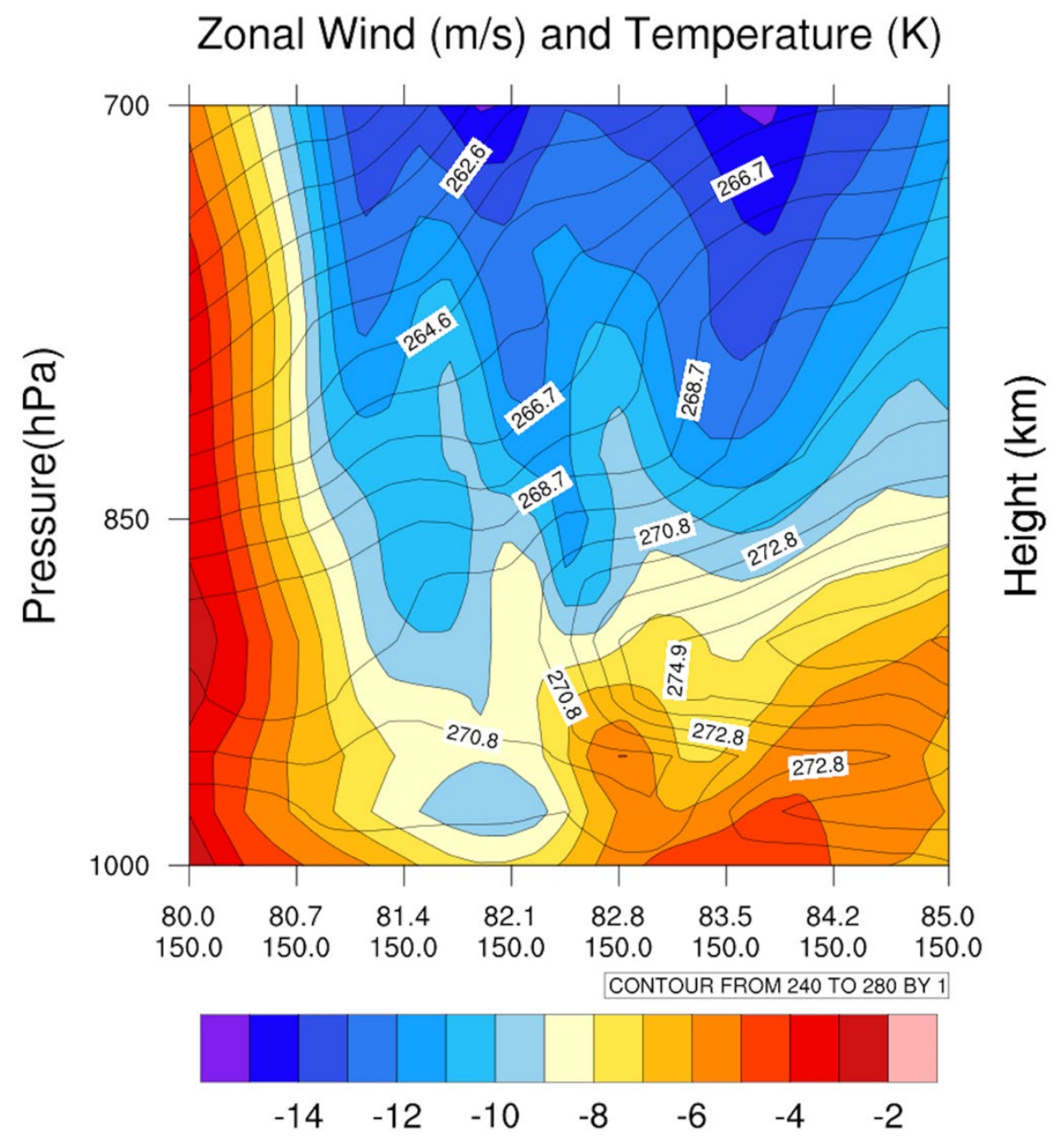


Figure 16. A longitudinal view of wind and temperature inversions associated with the $L \sqcup$ in Figure 15.

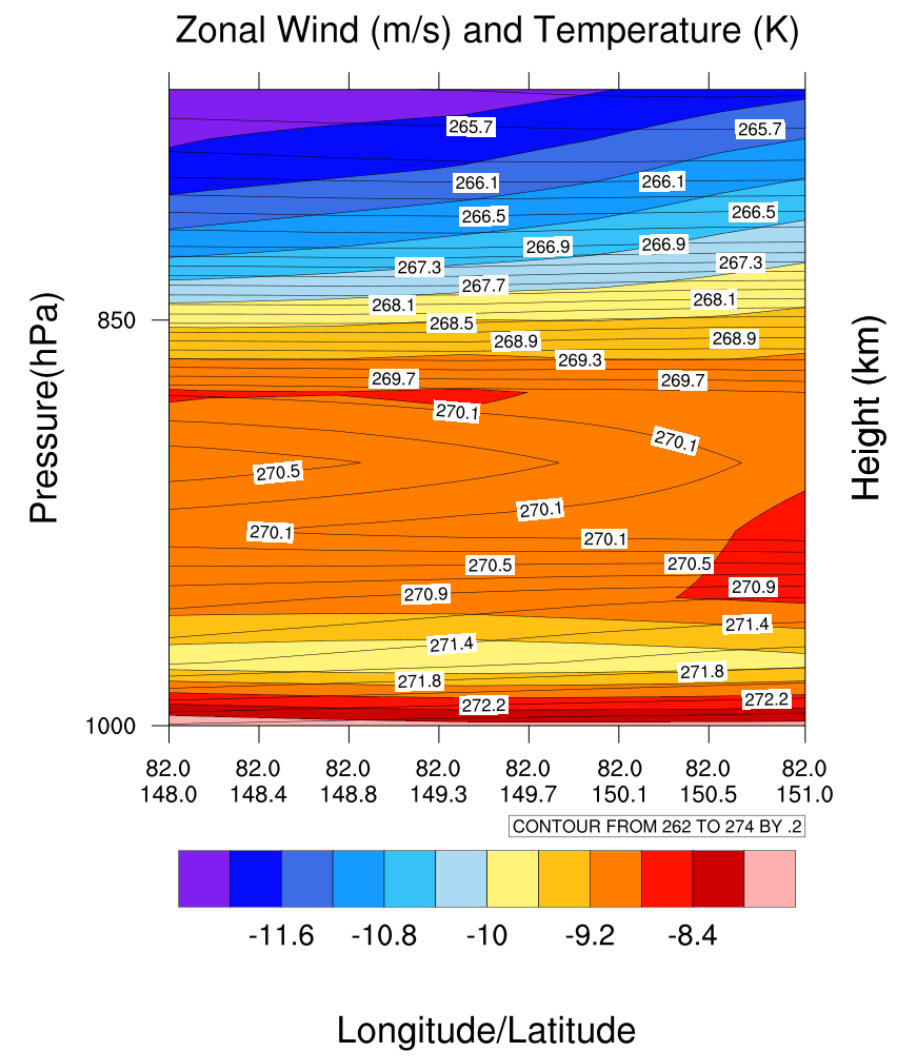

\subsection{Infrasound propagation}

In the following, we consider infrasound propagation calculations at two particular locations, which were identified based on the analyses in

Section 5.1. One of these is a cyclonic disturbance in the East Siberian Sea, and the other involves a strong katabatic flow in northern Greenland. We will see that propagation predictions differ dramatically between the two locations, as might be expected owing to the especially intense gradients encountered in these cases as can often occur in the Arctic. Although the 
ASRv2 provides fully 3-D fields, here we have simply extracted the profiles for the two locations and calculated the propagation under the idealization of a horizontal homogeneous atmosphere and ground surface.

\subsubsection{Polar low in East Siberian Sea}

As an example of infrasound propagation in the vicinity of a polar low, let us consider the atmospheric analysis on 6 January 2013, as was highlighted in Section 5.1, Figure 7-Figure 9. Vertical profiles were extracted from the PWRF simulation for a location in the East Siberian Sea, namely at latitude $73.372^{\circ}$ and longitude $150.555^{\circ}$. The profiles used in the infrasound propagation calculations are shown in Figure 17. Some characteristics of the major SSW event are also evident in the wind profile. The directions in these profiles are in the model coordinates (i.e., the PWRF polar stereographic projection), rather than Earth coordinates. The eastward wind component exhibits maxima at altitudes of about $5 \mathrm{~km}$ and $25 \mathrm{~km}$. The northward wind component exhibits a maximum at an altitude of about $20 \mathrm{~km}$ and reverses signs (from northward flow to southward) at about $27 \mathrm{~km}$. The temperature decreases with height through the troposphere up to $10-15 \mathrm{~km}$, at which altitude the increasing temperature layer (inversion) typical of the stratosphere takes over.

Figure 17. Vertical profiles for the East Siberian Sea, as extracted from the PWRF simulation at $\mathrm{OZ}$ on 6 January 2013 , at $73.372^{\circ}$ latitude and $150.555^{\circ}$ longitude. Height is $\mathrm{km}$ above ground (sea) level.
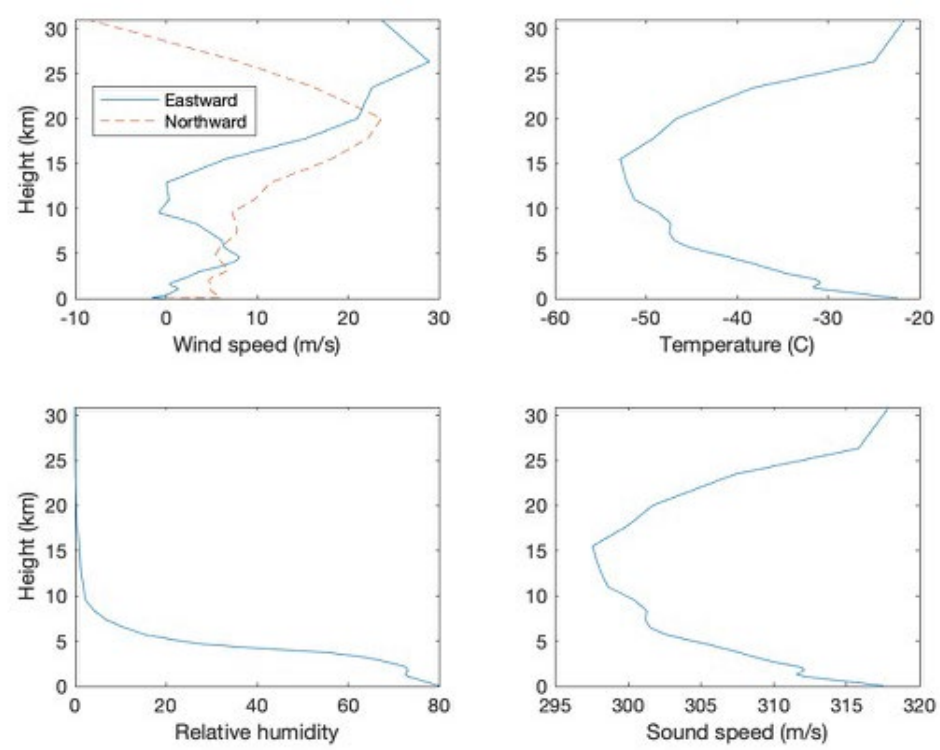
Figure 18 shows transmission loss (TL) calculations for a $5 \mathrm{~Hz}$ source. (TL is defined as the difference between the sound level observed at a particular location and the value that would be observed at a distance of 1 $\mathrm{m}$ from the source if it were radiating into free space.) The source height is set to $5 \mathrm{~m}$. These calculations were made with the WAPE as derived by the Padé $(1,1)$ approximation. The ground surface is assumed to be acoustically rigid, as is appropriate for water or ice. Also shown on the figure are ray paths as calculated with equations for a moving atmosphere, as described in Section 10.3 of Pierce (1981). These equations were integrated using a fourth-order Runge-Kutta method. In Figure 18, strong stratospheric returns are evident to the east and south; to the west and north, refraction is predominantly upward.

Figure 18. WAPE (Padé $(1,1)$ ) calculations for the East Siberian Sea polar low case, for a 5 Hertz $(\mathrm{Hz})$ source at $5 \mathrm{~m}$ height. In the top figure, westward propagation is to the left, eastward is to the right. In the bottom figure, southward propagation is to the left, northward to the right. The color scale represents transmission loss (TL) from $50 \mathrm{~dB}$ to $120 \mathrm{~dB}$. Gray lines are rays launched at $3^{\circ}$ increments.
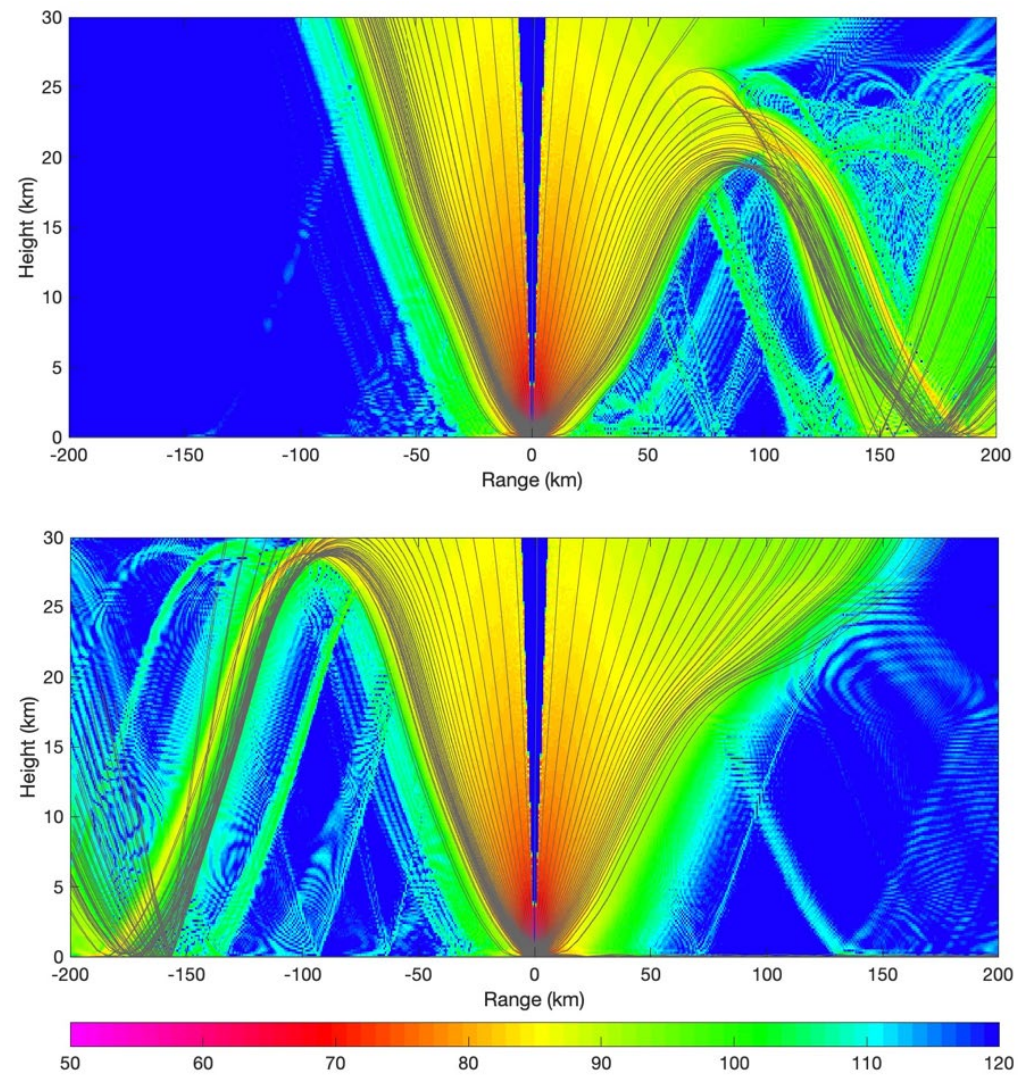
Figure 19 is similar to Figure 18, except that it shows the difference, in decibels (dB), between the WAPE calculations shown in Figure 18 and the corresponding NAPE calculations. Also shown on the plot are dashed lines at angles of $25^{\circ}, 50^{\circ}$, and $80^{\circ}$ and relative to the horizontal. At angles steeper than about $80^{\circ}$, the NAPE and WAPE are in agreement because neither has any propagating energy. At angles between $50^{\circ}$ and $80^{\circ}$, the WAPE predicts lower TLs because the NAPE has no propagating energy at these angles. Interestingly, at angles between roughly $25^{\circ}$ and $50^{\circ}$, the NAPE and WAPE are again in good agreement, apparently because there is minimal refraction at these angles. At angles less than about $25^{\circ}$, the TL difference has a very complicated behavior, probably because of multipath interference effects, for which the two approaches predict somewhat different phases in the various wave contributions.

Figure 19. Similar to Figure 18, except that the difference between the TLs predicted by the WAPE and NAPE calculations is shown. The color scale represents difference in TLs on a scale from $-20 \mathrm{~dB}$ to $20 \mathrm{~dB}$. Dashed lines are shown at angles of $25^{\circ}, 50^{\circ}$, and $80^{\circ}$ and relative to the horizontal. (Note that the horizontal and vertical scales differ so that the plotted angles of the lines differ from the physical angles.)
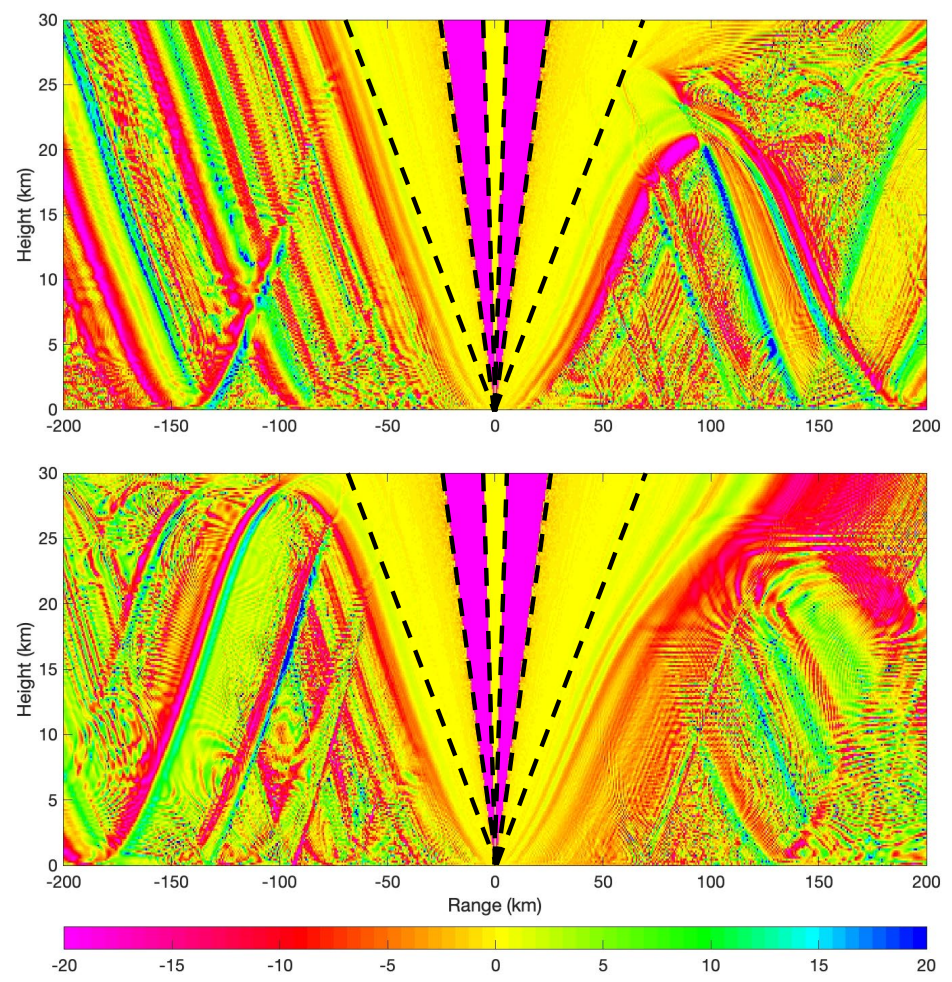

Figure 20 shows a close-up of the propagation to the east, near the range of $100 \mathrm{~km}$ and altitude of $25 \mathrm{~km}$. This close-up includes a well-defined caustic, that is, a region of crossing ray paths, which indicates strong 
focusing of sound energy. The caustic provides a useful feature for comparing the various calculation approaches. Three different combinations of PE calculations and ray traces are compared: the NAPE with ray tracing for a moving medium, the NAPE with ray tracing based on the ESSA, and the WAPE with ray tracing for a moving medium. The WAPE appears in perfect agreement with ray tracing (for a moving medium) regarding the caustic location. The NAPE, however, misses the location of the caustic by several kilometers. The location of the caustic agrees with the NAPE when the ESSA is employed for the ray tracing, which is to be expected since the ESSA is inherent to the NAPE. Taken together, these results provide solid evidence that the new Padé $(1,1)$ approximation is an improvement over the NAPE and ESSA.

Figure 20. Close-up of the eastward propagation in the vicinity of a caustic. Top left is the NAPE calculation with rays calculated using the correct equations for a moving medium. Top right is the NAPE calculation with rays calculated using the ESSA. Bottom is the WAPE calculation with rays calculated using the correct equations for a moving medium. The location of the caustic is indicated by the white ellipses.
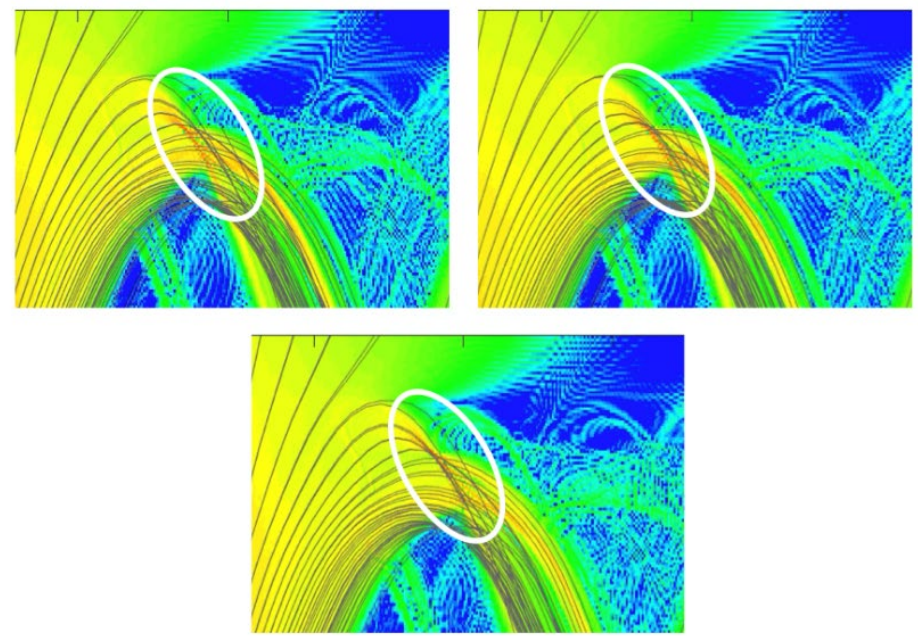

\subsubsection{Katabatic wind in northern Greenland}

Based on a visual analysis of the surface wind data from the ASRv2 data described in Section 5.1 (Figure 7-Figure 9), a katabatic wind was evident around the latitude/longitude of $\left(77.592^{\circ},-49.926^{\circ}\right)$, so this location was selected for more detailed analysis. The local terrain elevation is $2480 \mathrm{~m}$.

The profiles for this case are shown in Figure 21. The profiles indicate multiple temperature inversions, a near-surface katabatic flow, and associated impacts on the sound speed. The katabatic wind is evident as a 
strong LLJ, directed to the northwest, and a temperature inversion adjacent to the ground. Aloft, there is a strong westward jet at an altitude of about $20 \mathrm{~km}$, and a temperature lapse prevails up to this height.

Figure 21. Vertical profiles for northern Greenland, as extracted from the PWRF simulation at $\mathrm{OZ}$ on 6 Jan 2013, at $77.592^{\circ}$ latitude and $-49.926^{\circ}$ longitude. Height is in $\mathrm{km}$ above ground level, which is at $2480 \mathrm{~m}$.
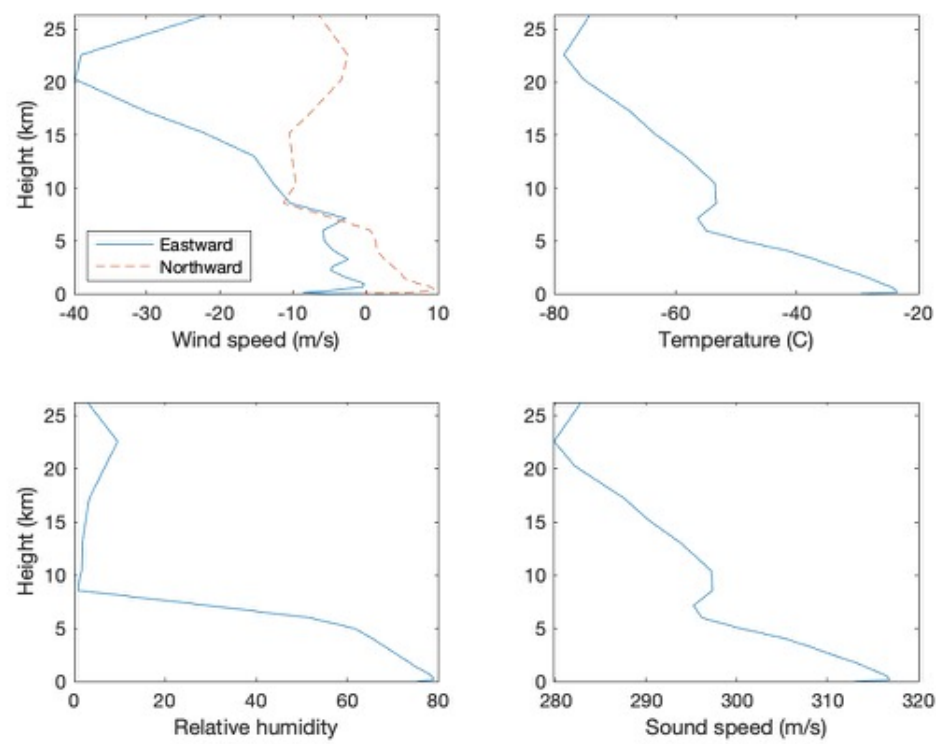

Figure 22 shows the TL calculations for a $5 \mathrm{~Hz}$ source at a height of $5 \mathrm{~m}$, using the WAPE as derived by the Padé $(1,1)$ approximation. A ground surface with properties characteristic of mature snow was used. Close examination of the figure reveals a surface duct at altitudes below $1 \mathrm{~km}$ or so, for propagation in the eastward and northward directions. This is a consequence of the katabatic wind. Above the surface duct, upward refraction prevails in all directions due to the strong lapse rate up to a height of $20 \mathrm{~km}$. 
Figure 22. WAPE (Padé $(1,1)$ ) calculations for the northern Greenland katabatic wind case, for a $5 \mathrm{~Hz}$ source at $5 \mathrm{~m}$ height. See caption to Figure 18 for further explanation.
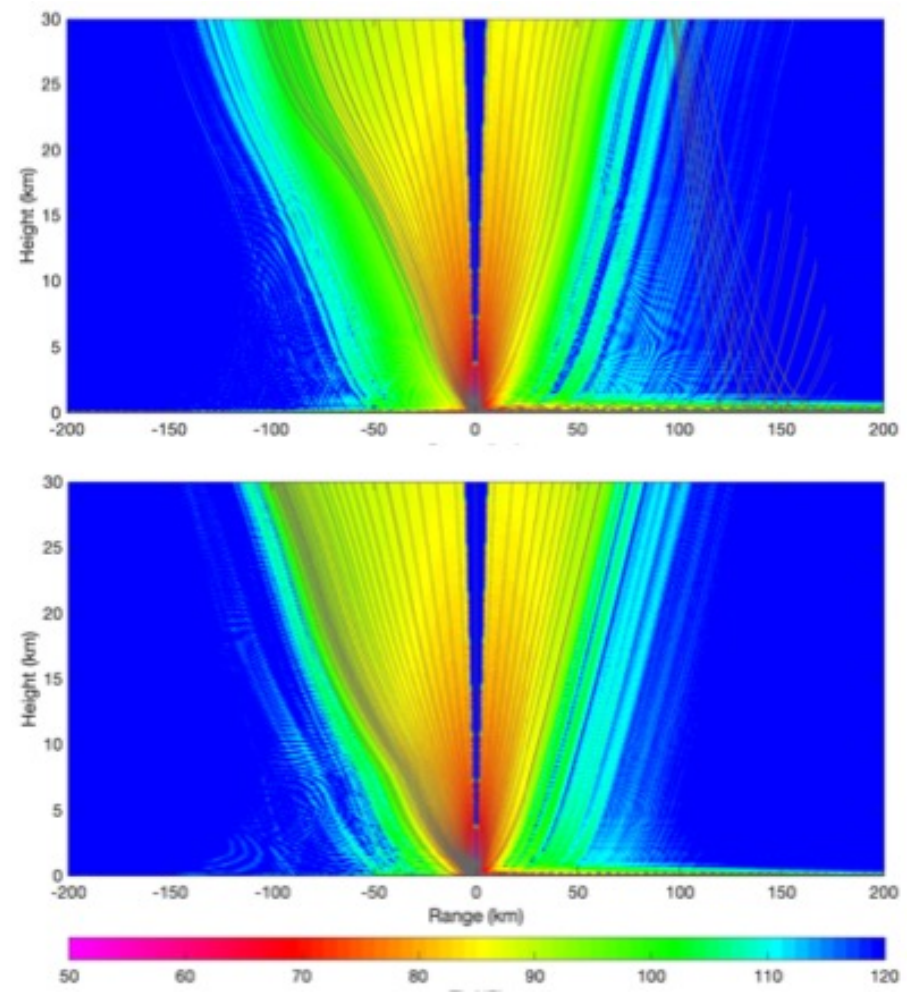

\subsection{Comparison of infrasound propagation at temperate and Arctic locations}

The influences of the Arctic environment on infrasound propagation become clearer when contrasted with more commonly studied environments. In this portion of the project, infrasound propagation was simulated for three distinct locations: Jackson, Mississippi $\left(32^{\circ} 17^{\prime} 56^{\prime \prime} \mathrm{N}\right.$, $\left.90^{\circ} 11^{\prime} 05^{\prime \prime} \mathrm{W}\right)$; Fairbanks, Alaska ( $\left.64^{\circ} 50^{\prime} 37^{\prime \prime} \mathrm{N}, 147^{\circ} 43^{\prime} 23^{\prime \prime} \mathrm{W}\right)$; and Danmarkshavn, Greenland $\left(76^{\circ} 46^{\prime} 8^{\prime \prime N}, 18^{\circ} 39^{\prime} 53^{\prime \prime} \mathrm{W}\right)$. These represent a temperate environment (Jackson), an inland Arctic environment (Fairbanks), and a coastal Arctic environment (Danmarkshavn).

The extreme northern latitudes present significant changes in meteorology over the annual cycle. To capture the range of conditions, four time periods of one week each during 2018 were chosen for analysis. The selected weeks surrounded the Spring Equinox (17-23 March 2018), Summer Solstice (18-24 June 2018), Fall Equinox (19-25 September 2018), and Winter Solstice (18-24 December 2018). The following sections describe the meteorological profiles and the infrasound propagation results for these time periods at each location. The final 
section compares results across the three locations and provides some insights into the similarities and differences between them.

\subsubsection{Atmospheric profiles}

For this study, meteorological data were obtained from archived radiosonde and ground station measurements. Two soundings were obtained for each location and day, at $\mathrm{OZ}$ and 12Z. Additionally, for each week, a mean profile was calculated for each of the two time periods. This resulted in up to 16 profiles for each week (14 individual soundings and 2 mean soundings) at each location. The radiosonde launch data were downloaded from the University of Wyoming, Department of Atmospheric Science (http://weather.uwyo.edu/upperair/sounding.html). The ground station information was pulled from the Weather Underground website, which hosts historical meteorological data for airports around the world (https://www.wunderground.com).

Although the soundings sample the atmosphere evenly in time, each launch samples a different set of altitudes. To ensure that the same altitudes are averaged, the radiosonde data were interpolated (using the Matlab interp1 function) to produce evenly sampled data with respect to altitude. The interpolated data were then averaged for each week to produce a mean profile. This procedure was applied independently for both the $\mathrm{oZ}$ and $12 \mathrm{Z}$ hours. Only radiosondes reaching above $25 \mathrm{~km}$ were included in the mean profiles. A Matlab script was developed to automate this process.

The sound speed in air was calculated from the temperature according to:

$$
c=20.05 \sqrt{T},
$$

where $T$ is the temperature in $\mathrm{K}$ and $c$ is in units of $\mathrm{m} / \mathrm{s}$. (Note that the impact of humidity on temperature is not included in the calculation.) The effective sound speed is determined as the sum of the actual sound speed and the horizontal wind component in the azimuthal propagation direction $\theta$; that is,

$$
c_{\mathrm{eff}}=c+W_{z} \cos \theta+W_{M} \sin \theta,
$$

where $\theta$ is given in the Cartesian convention ( $0 \mathrm{rad}$ to the east, positive counterclockwise), $W_{z}$ is the zonal (eastward) wind component, and $W_{M}$ is 
the meridional (northward) wind component. The equations relating wind speed and direction to the vector components are

$$
W_{z}=-W_{S} \sin \left(W_{D} \frac{\pi}{180}\right)
$$

and

$$
W_{M}=-W_{S} \cos \left(W_{D} \frac{\pi}{180}\right),
$$

where $W_{S}$ is the wind speed (m/s) and $W_{D}$ is the wind direction (deg) in the meteorological convention, namely $0^{\circ}$ when the wind is blowing from the north and positive clockwise (i.e., $90^{\circ}$ ) when the wind is blowing from the east).

Ground station data were obtained from the airports nearest the radiosonde launches. Since the radiosonde data begin around $100 \mathrm{~m}$ altitude, the ground station measurements serve as the anchor to the ground for the meteorological profiles. Simple linear interpolation was used to extend the profiles for temperature and wind to the ground. The interpolated and anchored meteorological profiles for each location are shown in the following figures. Each figure (Figure 23-Figure 34) shows the adiabatic sound speed profile $(\mathrm{m} / \mathrm{s})$, zonal (eastward), and meridional (northward) wind components $(\mathrm{m} / \mathrm{s}$ ) for each of the three locations at $12 \mathrm{Z}$ during each of the four 1-week-long sample periods. In each plot, the thin colored lines are profiles for single days, whereas the thick black lines are the mean profiles averaged over the week of interest.

The most consistent trend identified in the meteorological profiles is that the altitude of the tropopause (the boundary between the troposphere and stratosphere, where the temperature lapse of the troposphere gives way to increasing temperature in the stratosphere, and hence the sound-speed gradient tends to switch from negative to positive) is significantly lower at the northern latitude locations than at the temperate location. It is also lower at colder times of year. For Jackson during the week in June (Figure 28), the height of the transition is around $17 \mathrm{~km}$, while for Fairbanks during the week in December (Figure 33), it is around $9 \mathrm{~km}$. This behavior suggests that the so-called "zone of silence" characteristic of infrasound propagation out to ranges of $100 \mathrm{~km}-200 \mathrm{~km}$ (e.g., Pierce 1981) will be 
much more pronounced at temperate latitudes during the summer than at northern latitudes during the winter.

A further important trend in the profile data is the existence of a strong, persistent, ground-based temperature inversion in December for the Danmarkshavn and Fairbanks locations (Figure 32 and Figure 33, respectively), which manifests itself as a positive sound-speed gradient extending to an altitude of roughly $2 \mathrm{~km}$. As we will see shortly, this gradient can result in a strong surface duct and efficient long-range propagation. Since this feature is not present at the Jackson location (Figure 34), it appears to be an important characteristic of the wintertime atmosphere in the Arctic. The March and December profiles for Jackson also exhibit a high mean zonal wind speed (faster than $30 \mathrm{~m} / \mathrm{s}$ ), which is likely due to a southerly dip in the jet stream. The mean zonal wind speeds at the two northern latitude locations are relatively low during this interval. 
Figure 23. Radiosonde profiles for Danmarkshavn, Greenland, $12 Z$ March 2018. Shown are the sound speed (left), eastward (vx) wind component (center), and northward (vy) wind component (right). Each color is a different daily profile over the course of a single week. The thick black line is the mean profile for the week.
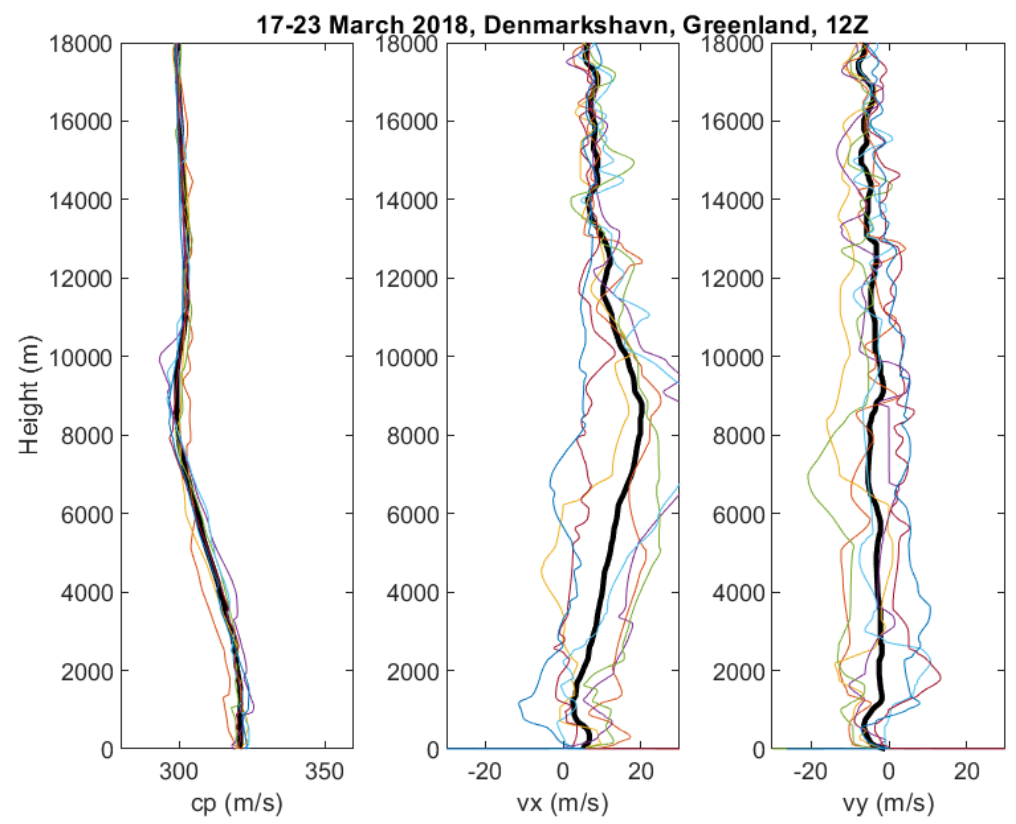

Figure 23.

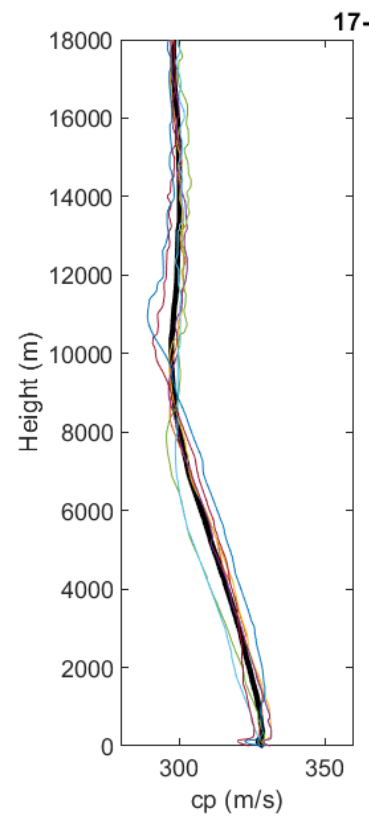

17-23 March 2018, Fairbanks, AK, $12 Z$

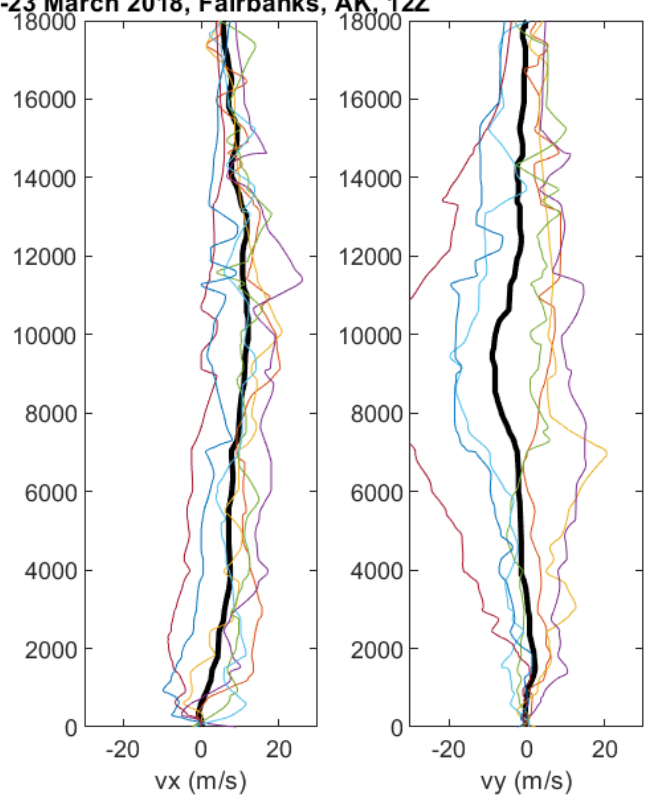


Figure 25. Radiosonde profiles for Jackson, MS, $12 Z$ March 2018. The profiles and colors are explained in Figure 23.

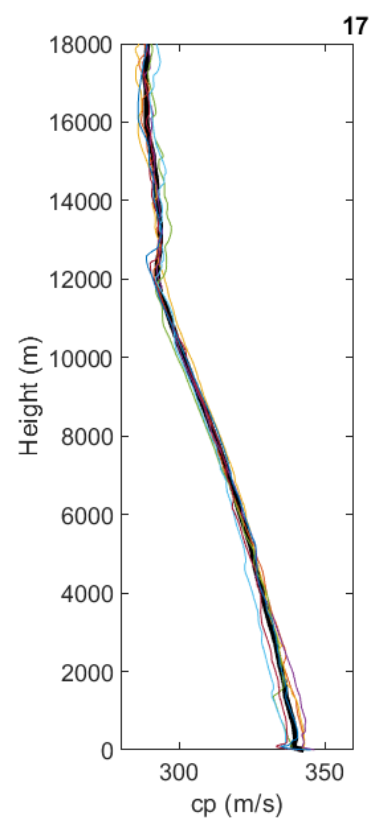

17-23 March 2018, Jackson, MS, $12 Z$
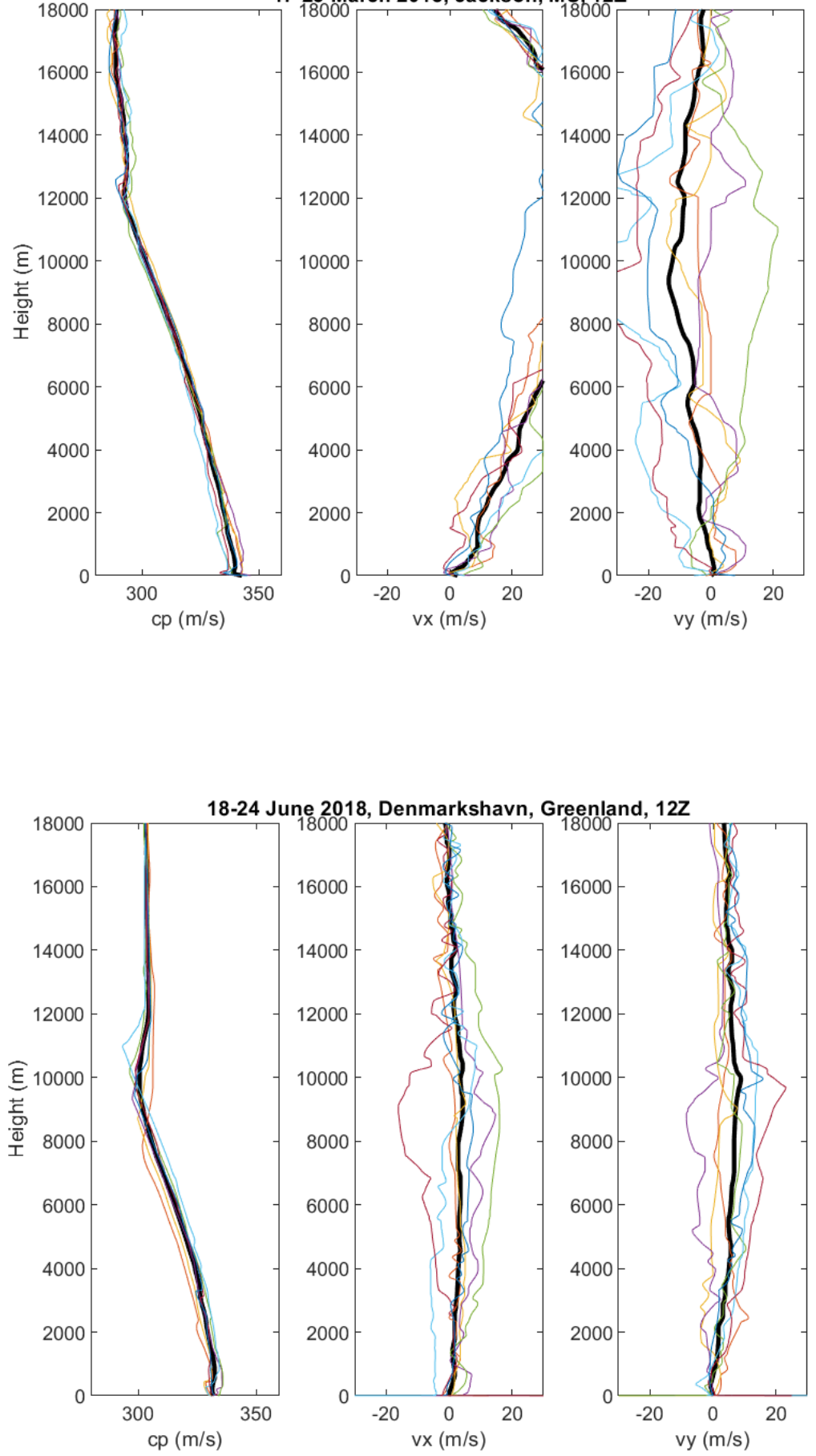
Figure 27. Radiosonde profiles for Fairbanks, AK, $12 Z$ June 2018. The profiles and colors are explained in Figure 23.

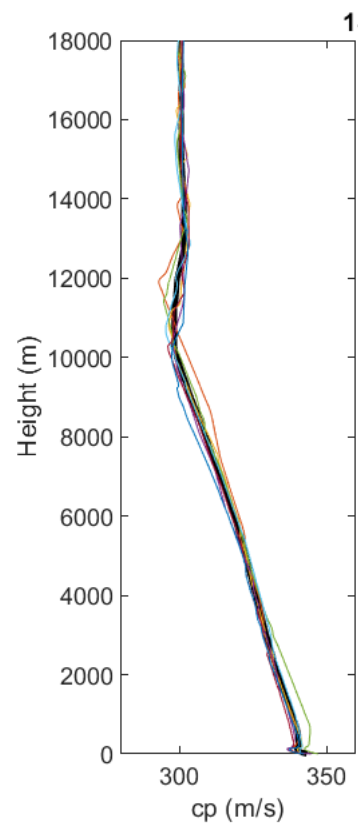

18-24 June 2018, Fairbanks, AK $12 Z$
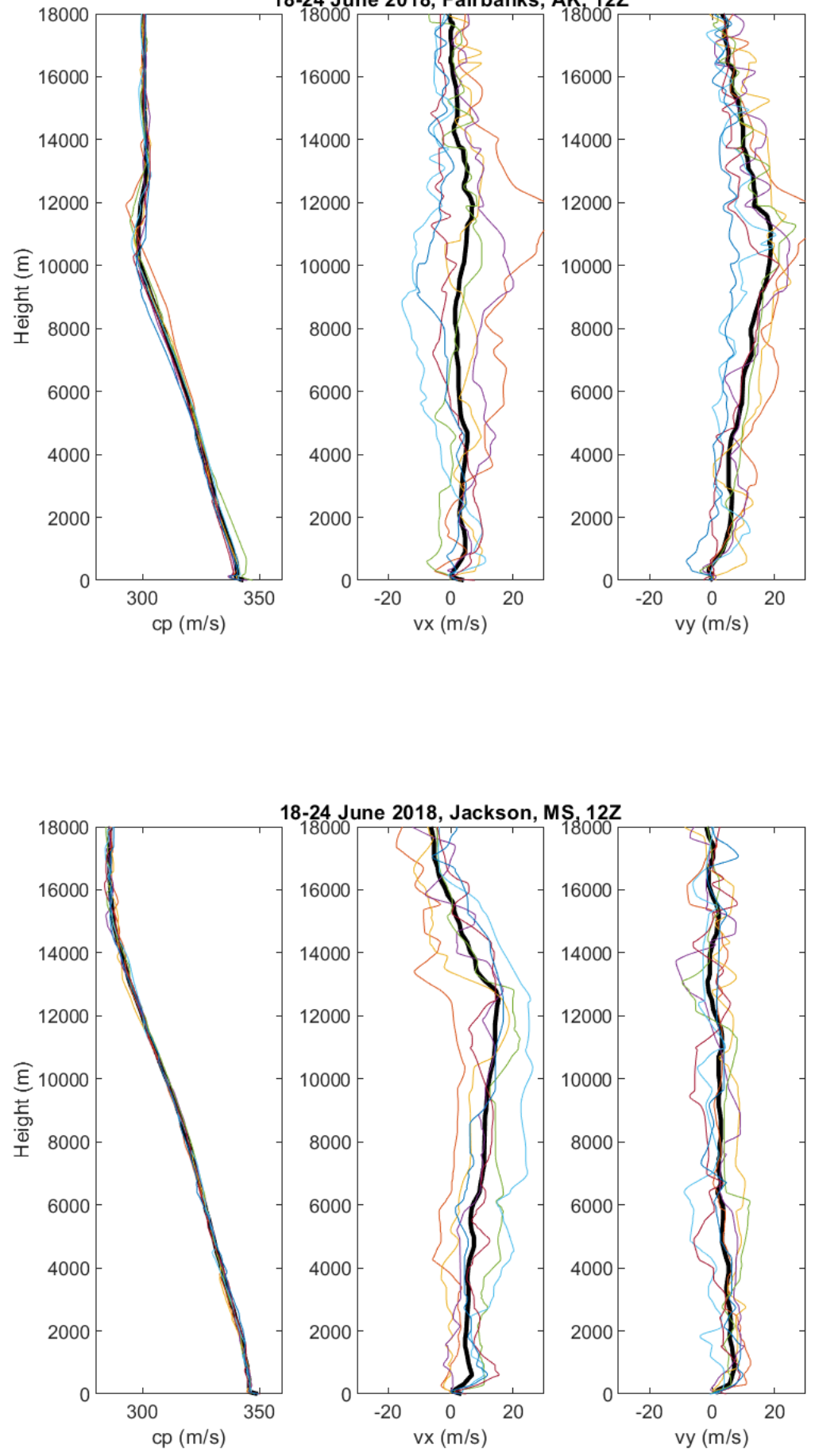

18-24 June 2018, Jackson, MS, $12 Z$

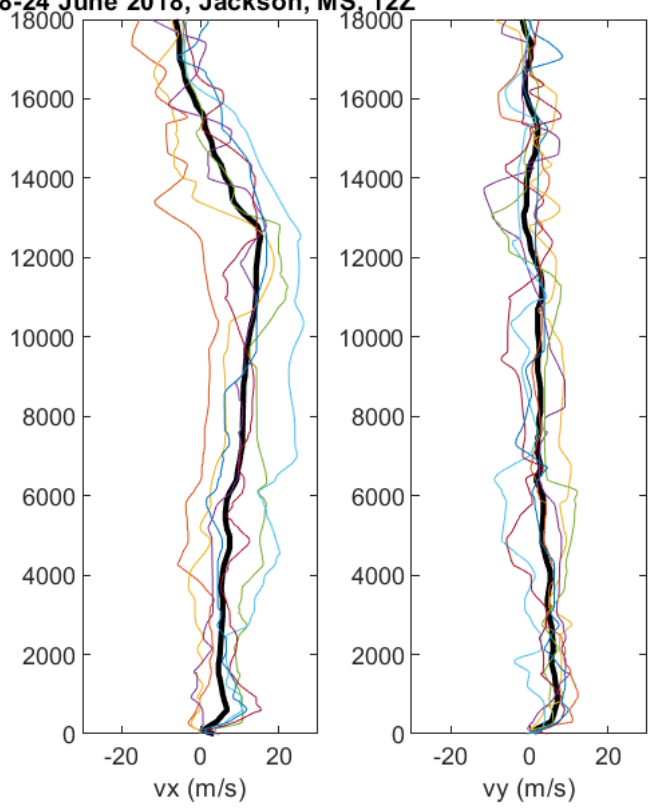


Figure 29. Radiosonde profiles for Danmarkshavn, Greenland, $12 Z$ September 2018. The profiles and colors are explained in Figure 23.
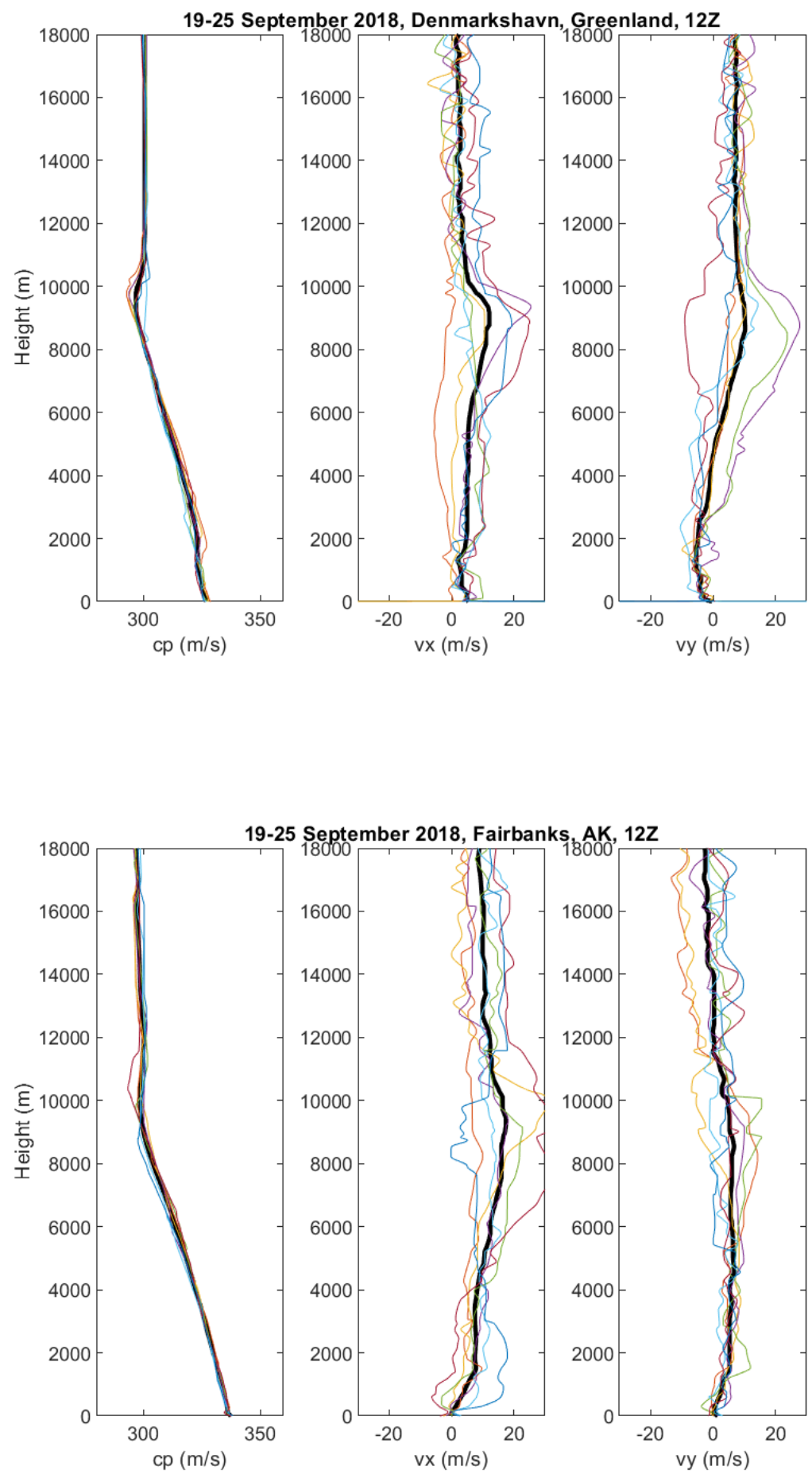

9-25 September 2018, Fairbanks, AK, $12 Z$

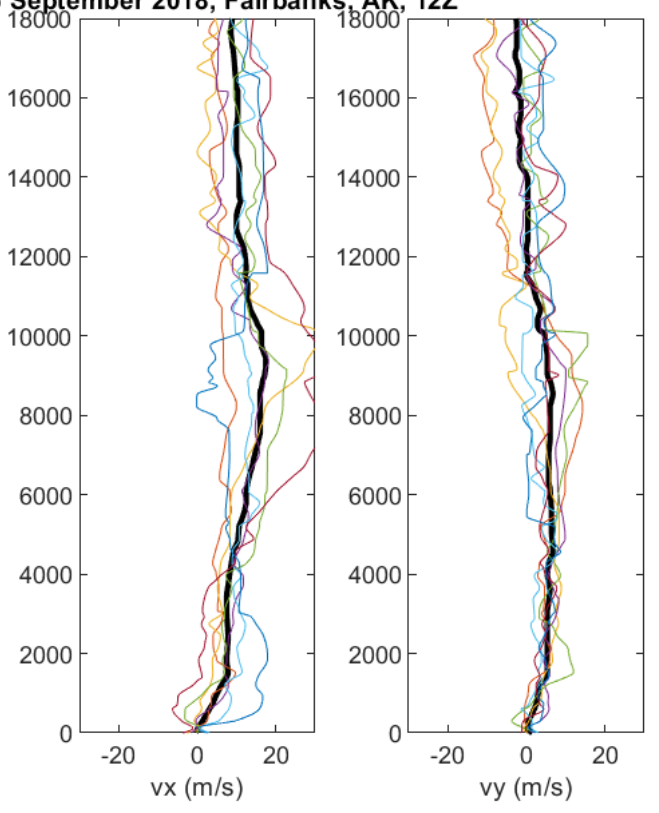


Figure 31. Radiosonde profiles for Jackson, MS, $12 Z$ September 2018. The profiles and colors are explained in Figure 23.
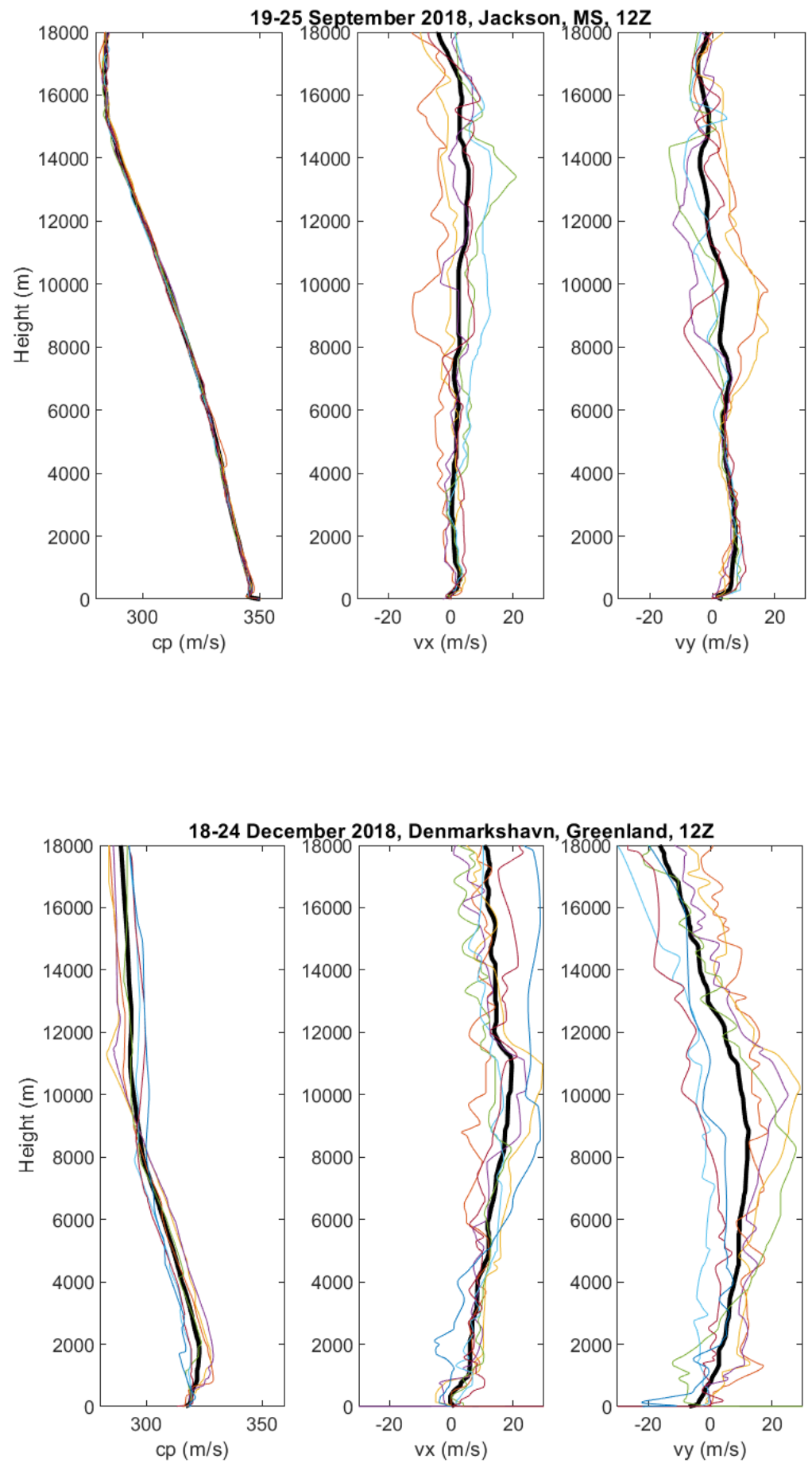
Figure 33. Radiosonde profiles for Fairbanks, AK, $12 Z$ December 2018. The profiles and colors are explained in Figure 23.

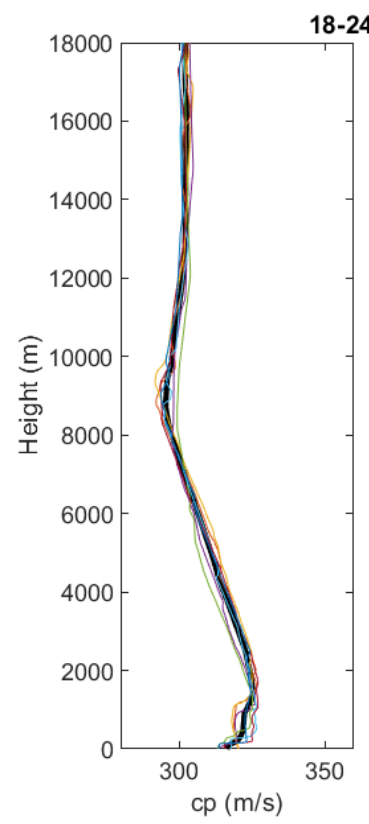

8-24 December 2018, Fairbanks, AK, $12 Z$
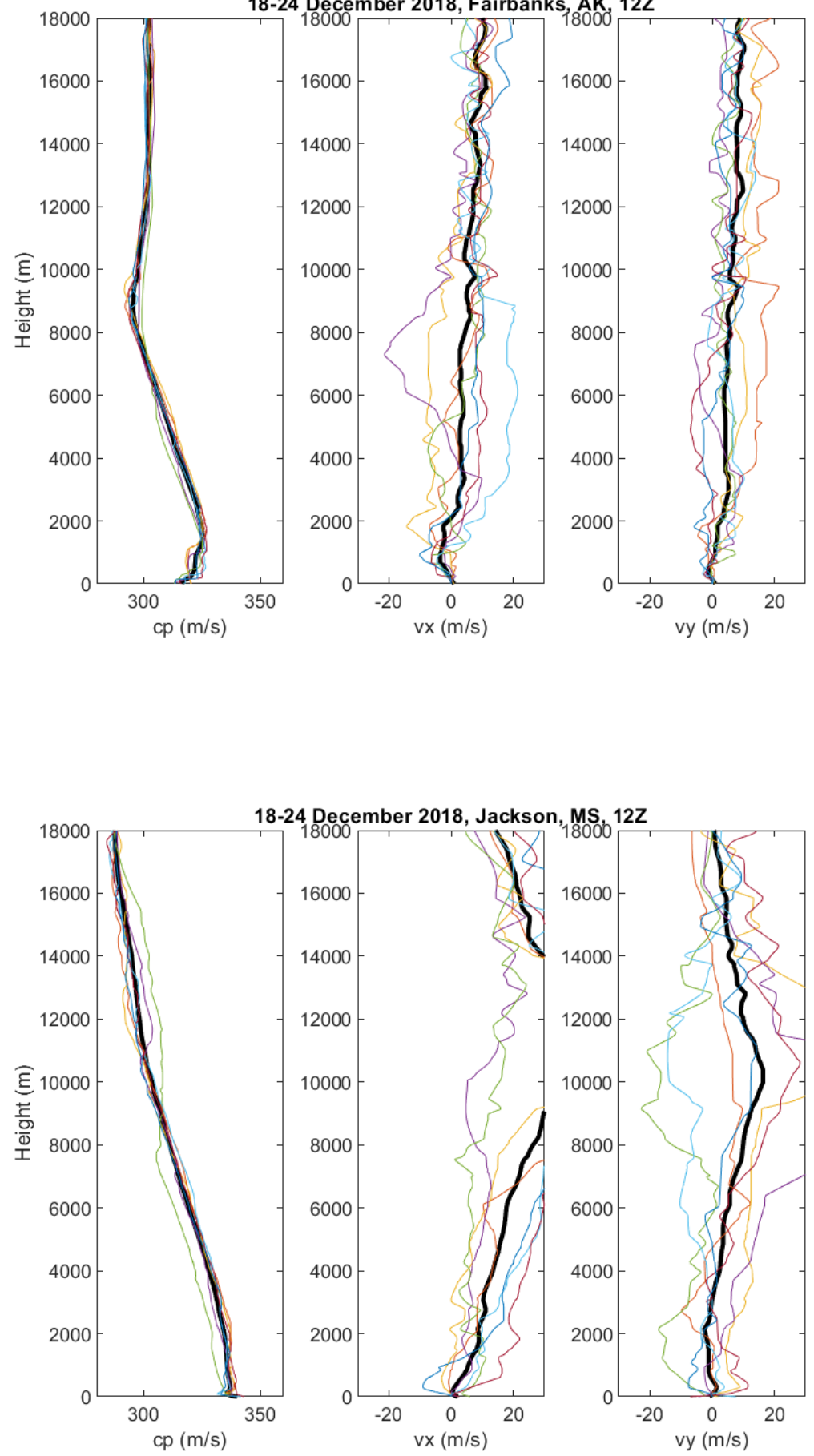

8-24 December 2018, Jackson, MS, $12 Z$

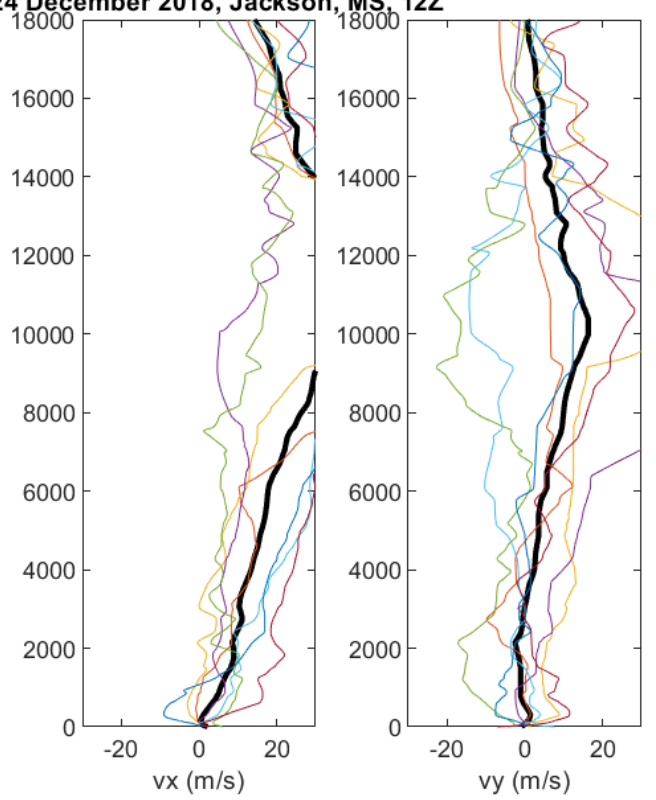




\subsubsection{Infrasound propagation simulations}

A wide-angle Crank-Nicholson PE implementation was selected for this portion of the study. This set of simulations was performed simultaneously with development of the EWAPE; because the EWAPE was unavailable at the time of these calculations, ESSA was used. Because this portion of the project was directly investigating the influence of the unique meteorology on infrasound propagation, the ground was assumed to be flat and rigid rather than including more location-specific terrain and topography. Three (3) frequencies (2, 4, and $8 \mathrm{~Hz}$ ) and 12 propagation directions (every $30^{\circ}$ ) were considered. Due to constraints on the maximum altitude measured with the radiosondes, a maximum calculation altitude of $15 \mathrm{~km}$ and horizontal range of $120 \mathrm{~km}$ was selected. A drawback of this limitation is that it potentially misses stratospheric returns such as those shown to the east and south in Figure 18.

In this report, the weeks surrounding the summer and winter solstices and spring and fall equinoxes, at $12 Z$, are shown for each location for propagation of a $4 \mathrm{~Hz}$ signal in each of the four cardinal directions. (The other frequencies and propagation directions mentioned in the preceding paragraph produced similar results and are not included for brevity.) In the following figures, the top plot shows propagation to the west (left) and east (right), and the bottom plot shows propagation to the south (left) and north (right). The line plots are all taken just above the ground boundary. Simulations are presented in chronological order (winter, spring, summer, and fall). This set of figures accomplishes two different things. First, the line plots provide an indication of the variability in propagation during the weeks of interest, as well as providing some insights into the effectiveness of using a mean profile for predictions. Second, the 2-D plots visualize the refractive properties of the mean profiles in range versus distance slices for each of the four propagation directions.

\subsubsection{Discussion}

Examining the line plots of TL versus distance (Figure 35, Figure 37, Figure 39, Figure 41, Figure 43, Figure 45, Figure 47, Figure 49, Figure 51, Figure 53, Figure 55, and Figure 57), we can see that the mean profiles adequately capture the average propagation characteristics in a given direction. However, they do not capture the substantial day-to-day variability. The day-to-day variability is large and most likely due to 
variations in the synoptic weather conditions (i.e., the passages of storm systems and fronts).

All three locations exhibit significant variability in the propagation characteristics. The temperate location (Jackson, MS) has a higher number of strongly upward-refracting conditions compared to the Arctic conditions (Alaska and Greenland). However, these simulations were (1) performed using a standard wide-angle PE and (2) only considered propagation out to $120 \mathrm{~km}$ and vertical profiles to an altitude of $15 \mathrm{~km}$. As can be seen from the profiles in Section 5.2, atmospheric phenomena occurring at higher altitudes, within the stratosphere, can strongly impact the propagation, particularly for distances exceeding roughly $100 \mathrm{~km}$.

The propagation characteristics at the Danmarkshavn and Fairbanks locations in December (Figure 54 and Figure 56, respectively), and to a somewhat lesser extent in March, are particularly notable. Recall from Section 5.3.1 that these locations exhibit a surface-based, positive soundspeed gradient at this time. The impact on the propagation is quite evident. Strong surface ducting occurs in all four directions in Fairbanks. In Danmarkshavn, the wind plays a somewhat greater role, with ducting in three of four propagation directions. Surface-based ducting does occur intermittently at other times of year at the northern latitude locations (Fairbanks and Danmarkshavn) and at the temperate latitude location (Jackson). In those cases, however, the persistent positive sound-speed gradient of the northern latitude wintertime is less evident, and ducting is attributable to both sound-speed and wind gradients, sometimes in combination. The wind-driven ducting is evident when the propagation direction and wind coincide.

For Jackson in March (Figure 40), there is a particularly deep and persistent duct, extending up to an altitude of about $5 \mathrm{~km}$, for eastward propagation. A similar feature is evident, although not so deep, for Jackson in December (Figure 58). Since this feature appears only in one direction, it must be caused by the strong wind-velocity gradients evident in the meteorological profiles, which are apparently linked to the jet stream. 
Figure 35. TL versus distance, 17-23 March 2018, 12Z, Danmarkshavn, Greenland. Thick black line represents the mean profile for the week, all other lines represent individual days. Top plot shows propagation to the west (left) and east (right). Bottom plot shows propagation to the south (left) and north (right). Frequency is $4 \mathrm{~Hz}$.
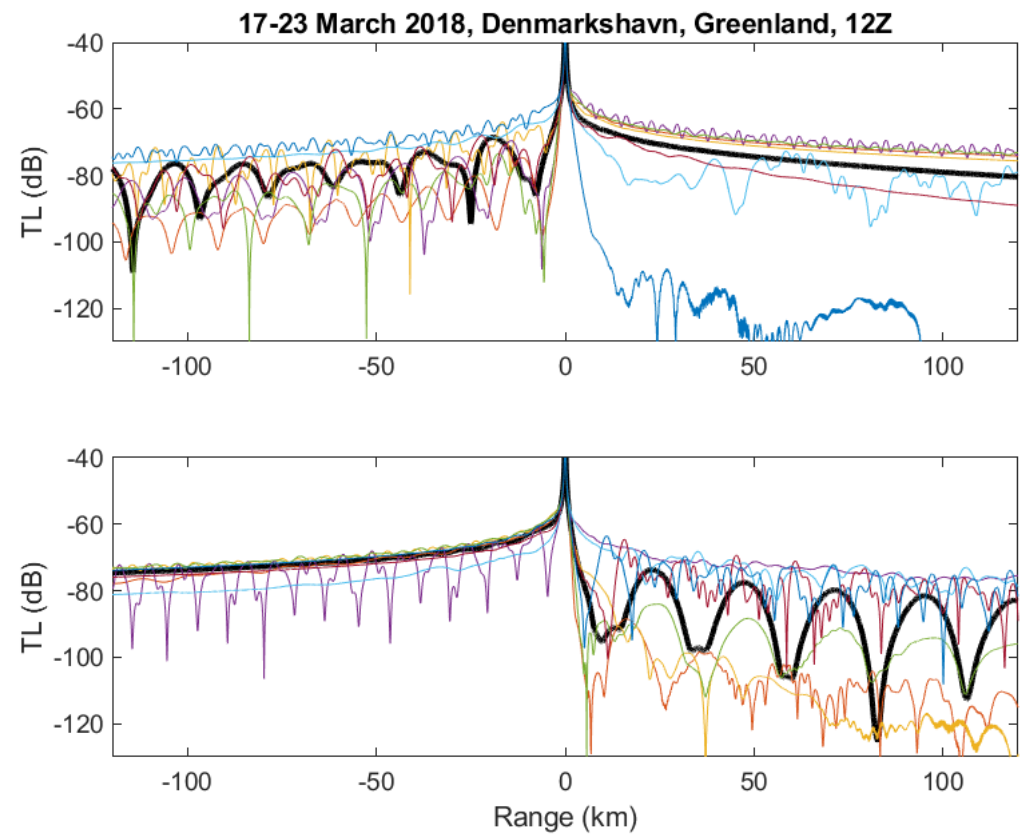

Figure 36. TL as a function of range and altitude, mean profile for the 17-23 March 2018 time period, 12Z, in Danmarkshavn, Greenland. Top plot shows propagation to the west (left) and east (right). Bottom plot shows propagation to the south (left) and north (right). Frequency is $4 \mathrm{~Hz}$.
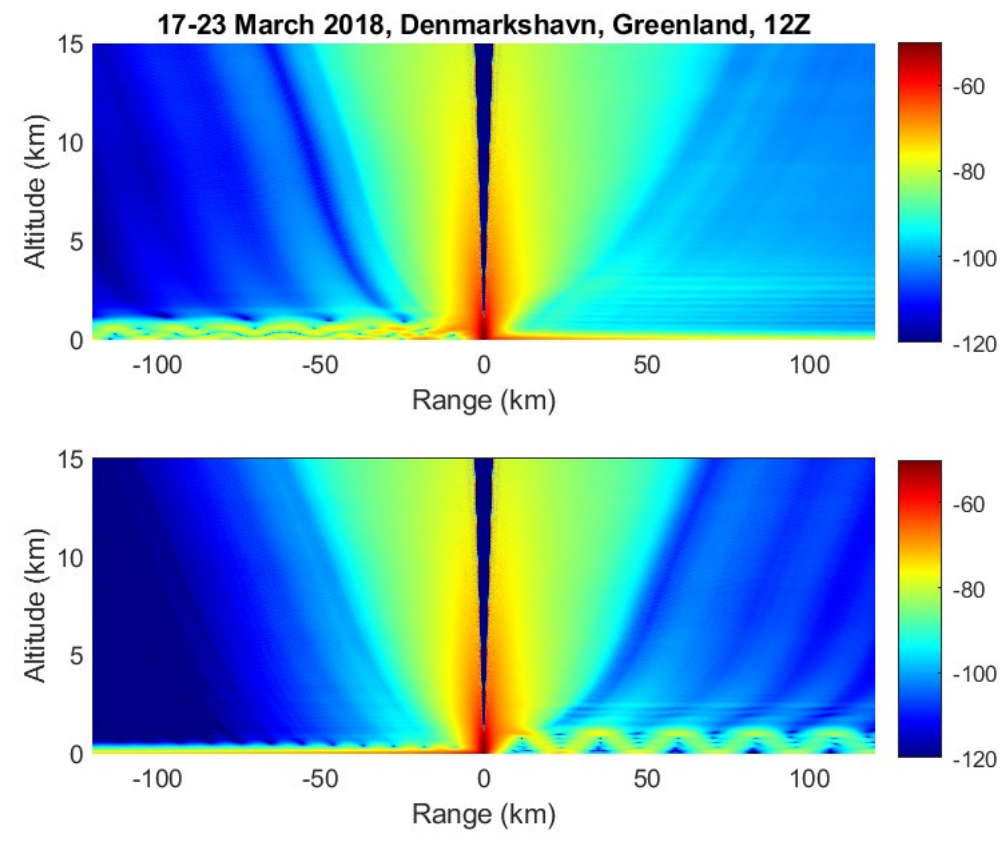
Figure 37. TL versus distance, 17-23 March 2018, 12Z, Fairbanks, AK. Thick black line represents the mean profile for the week, all other lines represent individual days. Top plot shows propagation to the west (left) and east (right). Bottom plot shows propagation to the south (left) and north (right). Frequency is $4 \mathrm{~Hz}$.
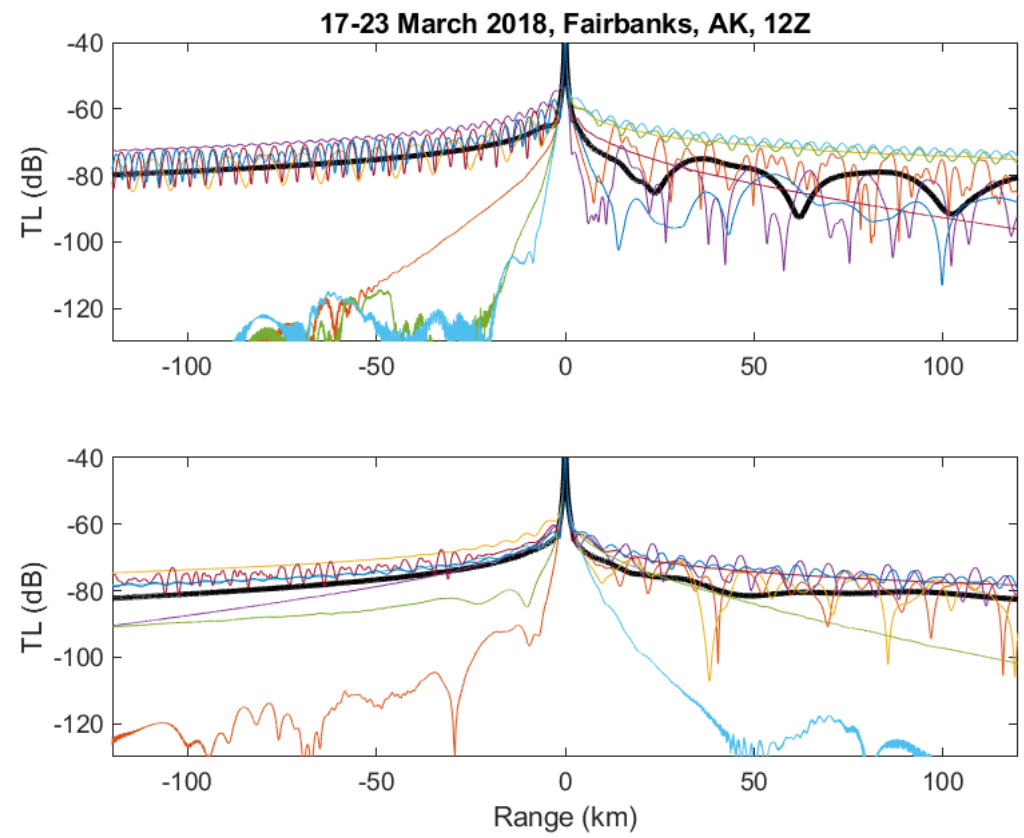

Figure 38. TL as a function of range and altitude, mean profile for the 17-23 March 2018 time period, 12Z, in Fairbanks, AK. Top plot shows propagation to the west (left) and east (right). Bottom plot shows propagation to the south (left) and north (right). Frequency is $4 \mathrm{~Hz}$.
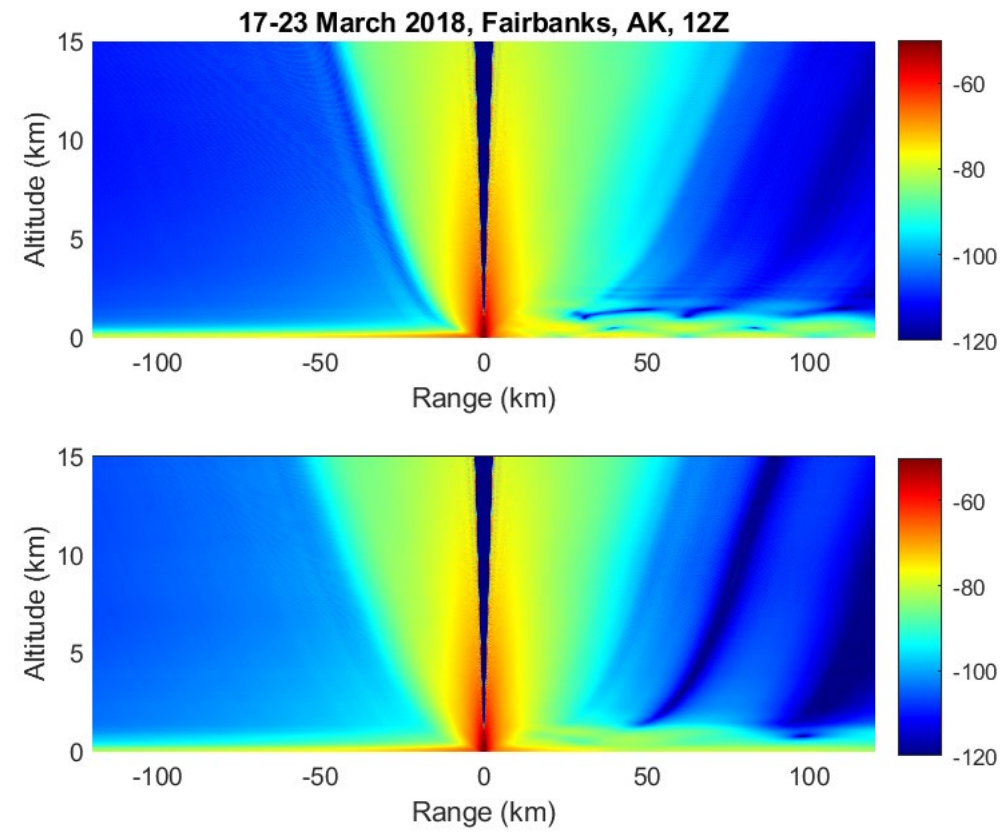
Figure 39. TL versus distance, 17-23 March 2018, 12Z, Jackson, MS. Thick black line represents the mean profile for the week, all other lines represent individual days. Top plot shows propagation to the west (left) and east (right). Bottom plot shows propagation to the south (left) and north (right). Frequency is $4 \mathrm{~Hz}$.
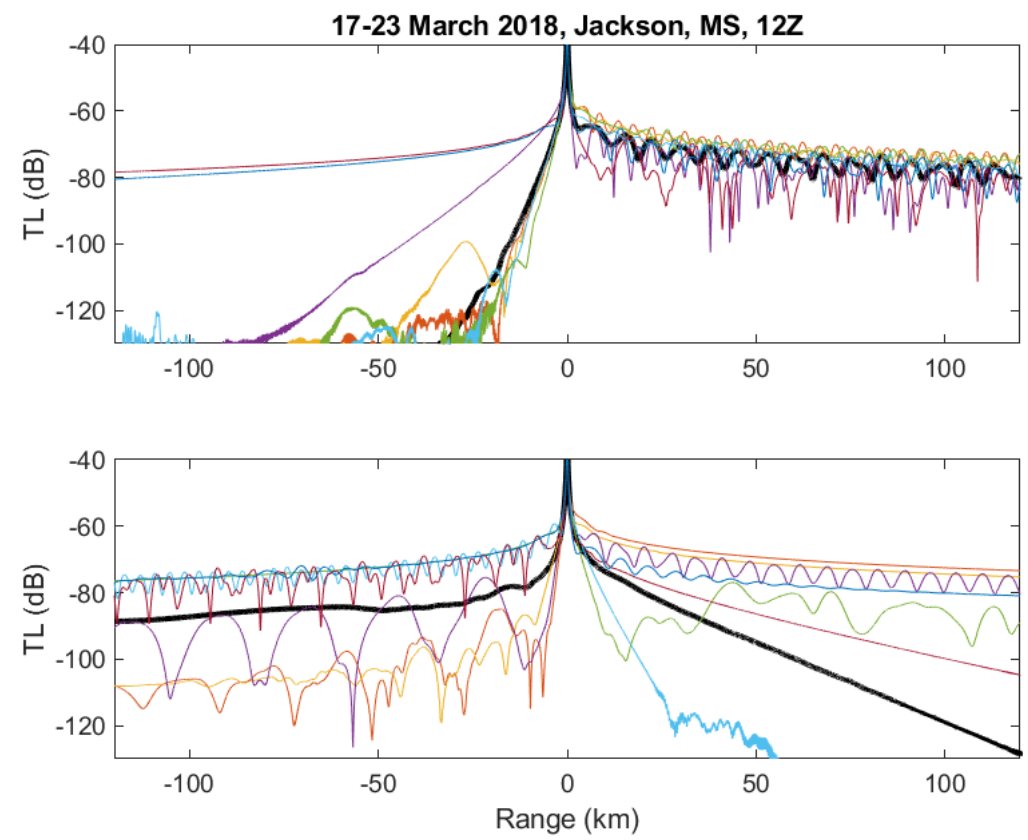

Figure 40. TL as a function of range and altitude, mean profile for the 17-23 March 2018 time period, 12Z, in Jackson, MS. Top plot shows propagation to the west (left) and east (right). Bottom plot shows propagation to the south (left) and north (right). Frequency is $4 \mathrm{~Hz}$.
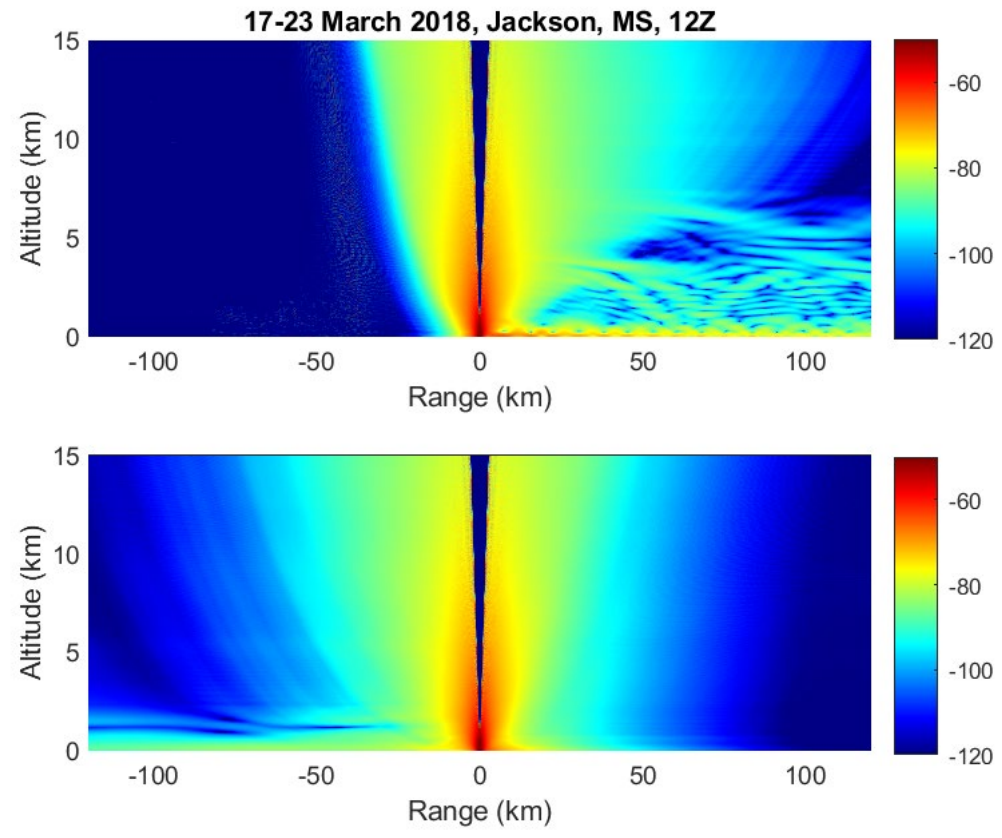
Figure 41. TL versus distance, 18-24 June 2018, 12Z, Danmarkshavn, Greenland. Thick black line represents the mean profile for the week, all other lines represent individual days. Top plot shows propagation to the west (left) and east (right). Bottom plot shows propagation to the south (left) and north (right). Frequency is $4 \mathrm{~Hz}$.
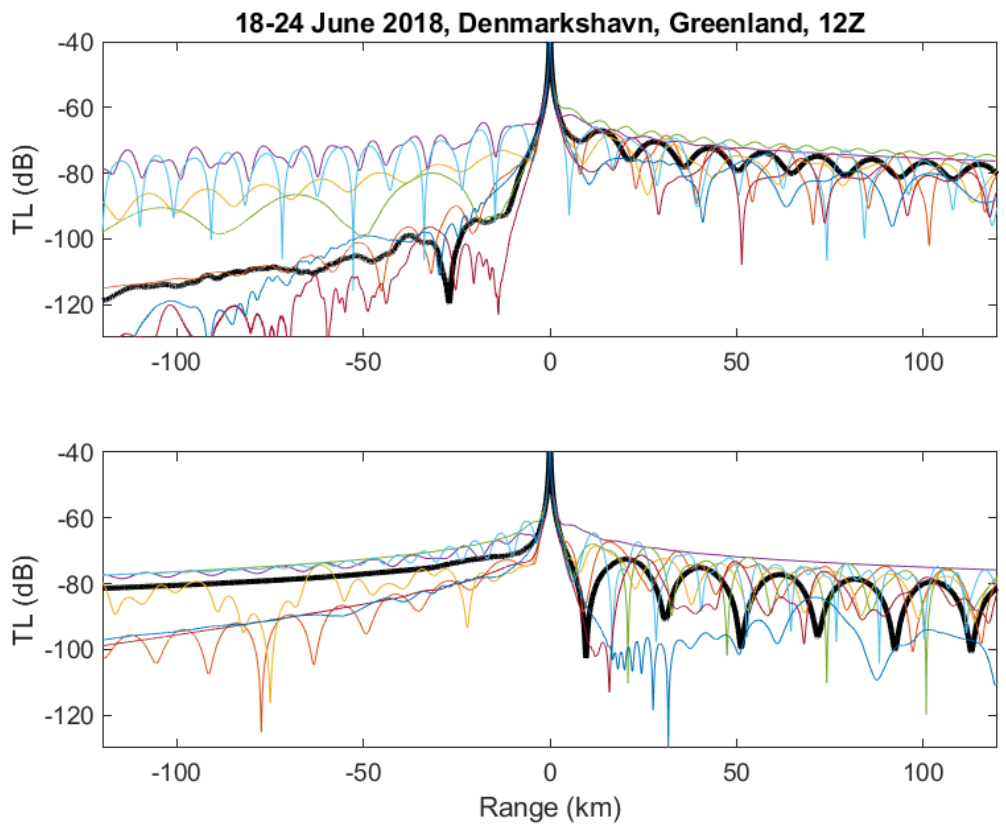

Figure 42. TL as a function of range and altitude, mean profile for the 18-24 June 2018 time period, 12Z, in Danmarkshavn, Greenland. Top plot shows propagation to the west (left) and east (right). Bottom plot shows propagation to the south (left) and north (right). Frequency is 4 $\mathrm{Hz}$.
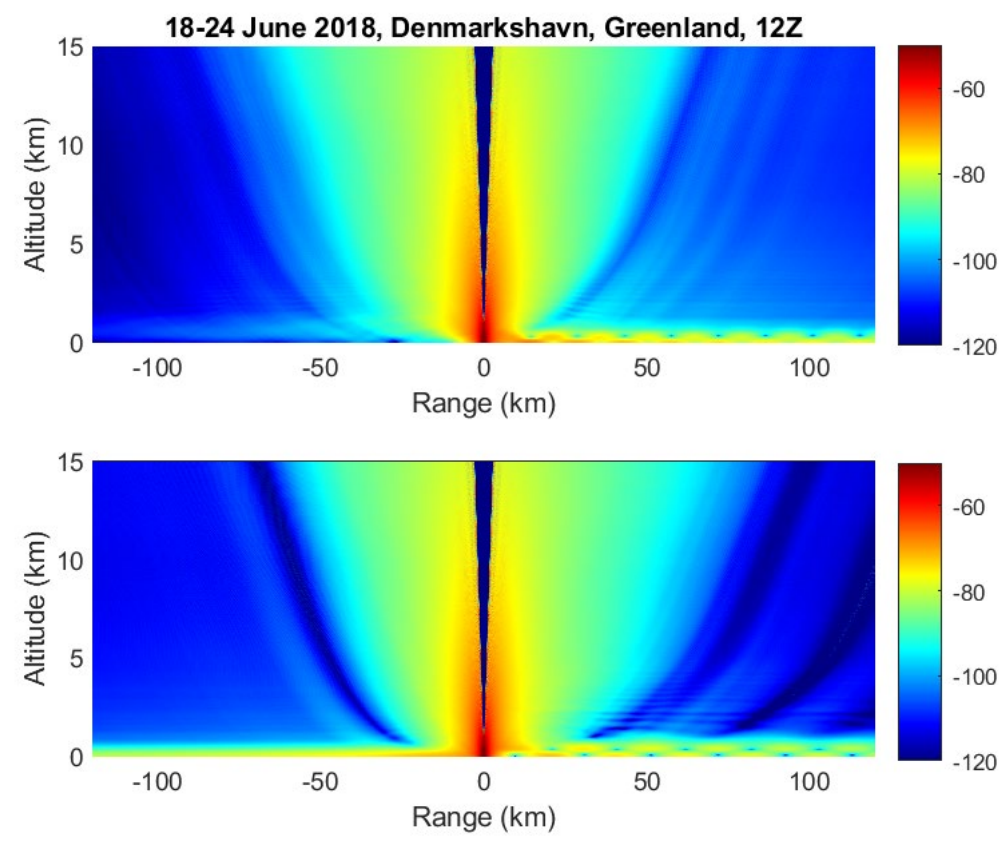
Figure 43. TL versus distance, 18-24 June 2018, 12Z, Fairbanks, AK. Thick black line represents the mean profile for the week, all other lines represent individual days. Top plot shows propagation to the west (left) and east (right). Bottom plot shows propagation to the south (left) and north (right). Frequency is $4 \mathrm{~Hz}$.
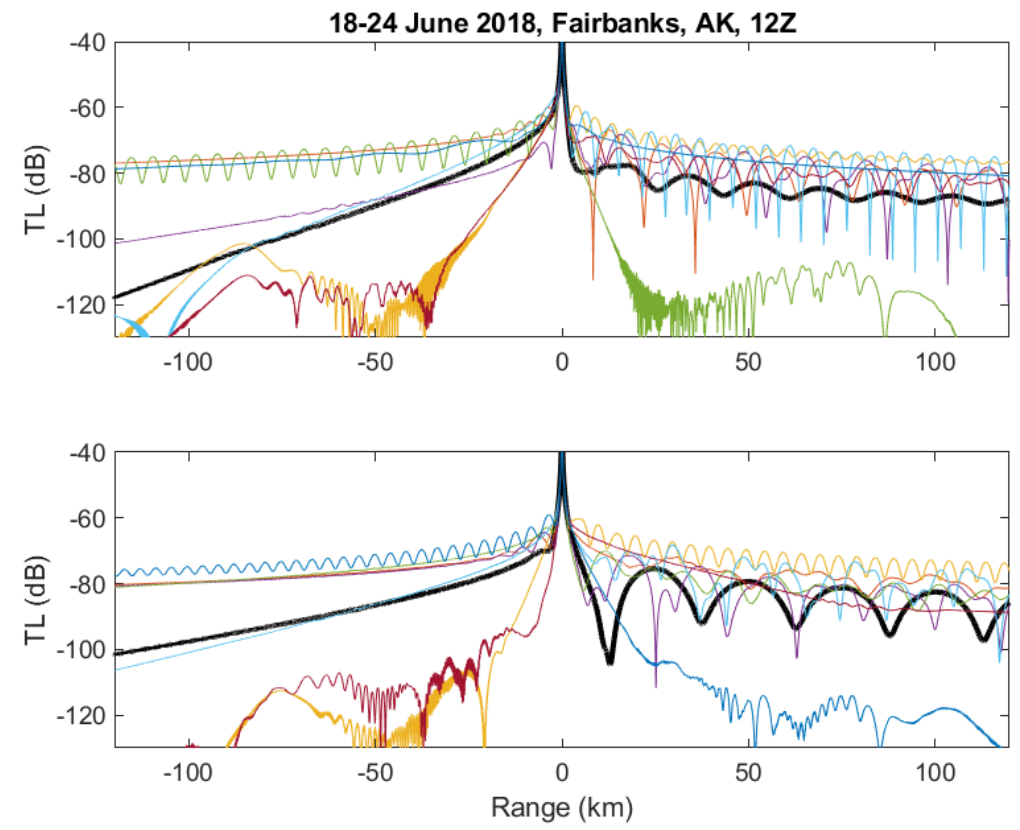

Figure 44. TL as a function of range and altitude, mean profile for the 18-24 June 2018 time period, 12Z, in Fairbanks, AK. Top plot shows propagation to the west (left) and east (right).

Bottom plot shows propagation to the south (left) and north (right). Frequency is $4 \mathrm{~Hz}$.
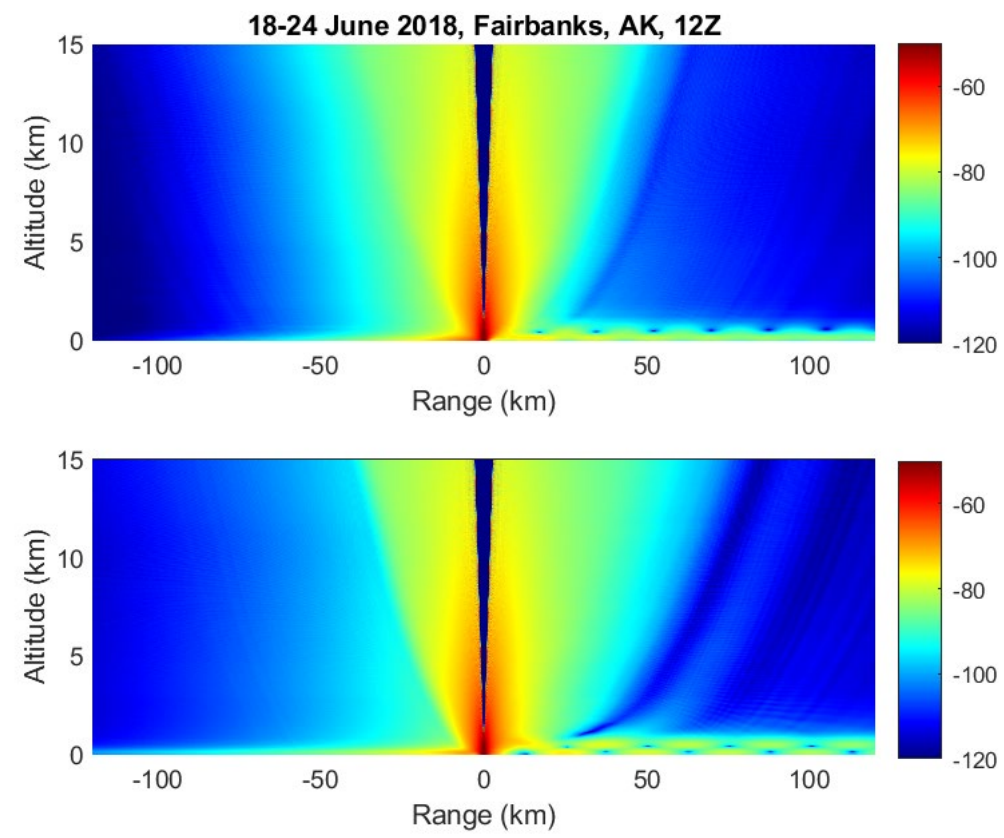
Figure 45. TL versus distance, 18-24 June 2018, 12Z, Jackson, MS. Thick black line represents the mean profile for the week, all other lines represent individual days. Top plot shows propagation to the west (left) and east (right). Bottom plot shows propagation to the south (left) and north (right). Frequency is $4 \mathrm{~Hz}$.
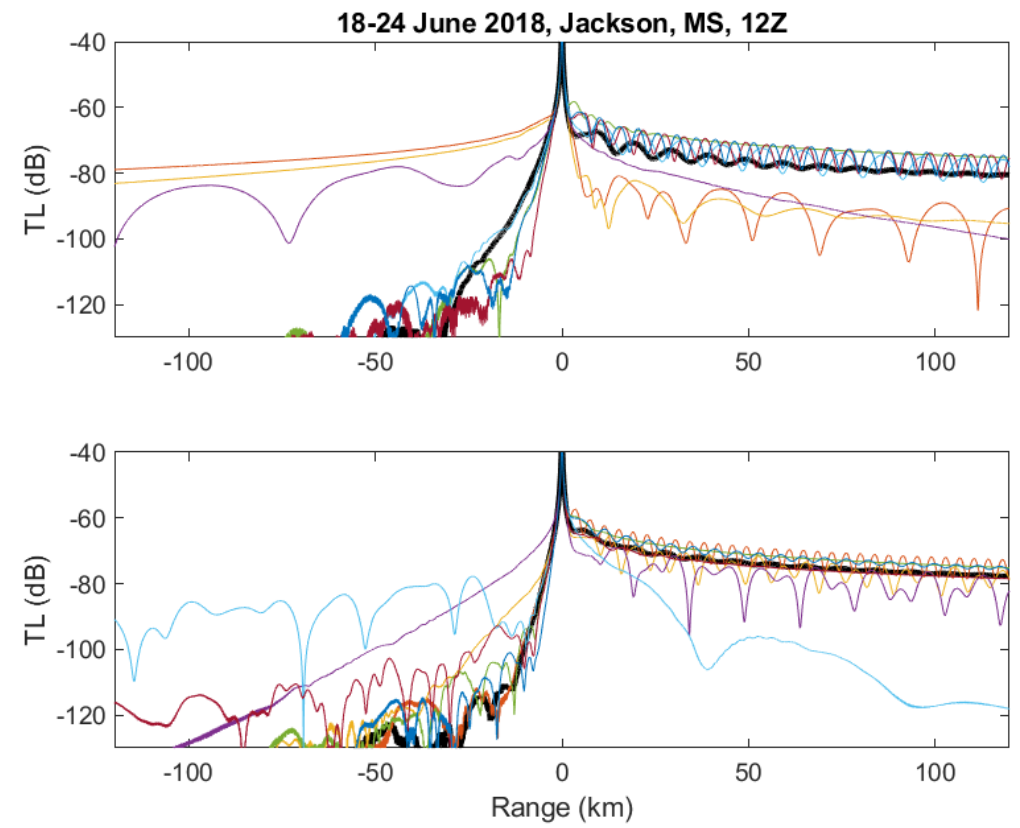

Figure 46. TL as a function of range and altitude, mean profile for the 18-24 June 2018 time period, 12Z, in Jackson, MS. Top plot shows propagation to the west (left) and east (right).

Bottom plot shows propagation to the south (left) and north (right). Frequency is $4 \mathrm{~Hz}$.
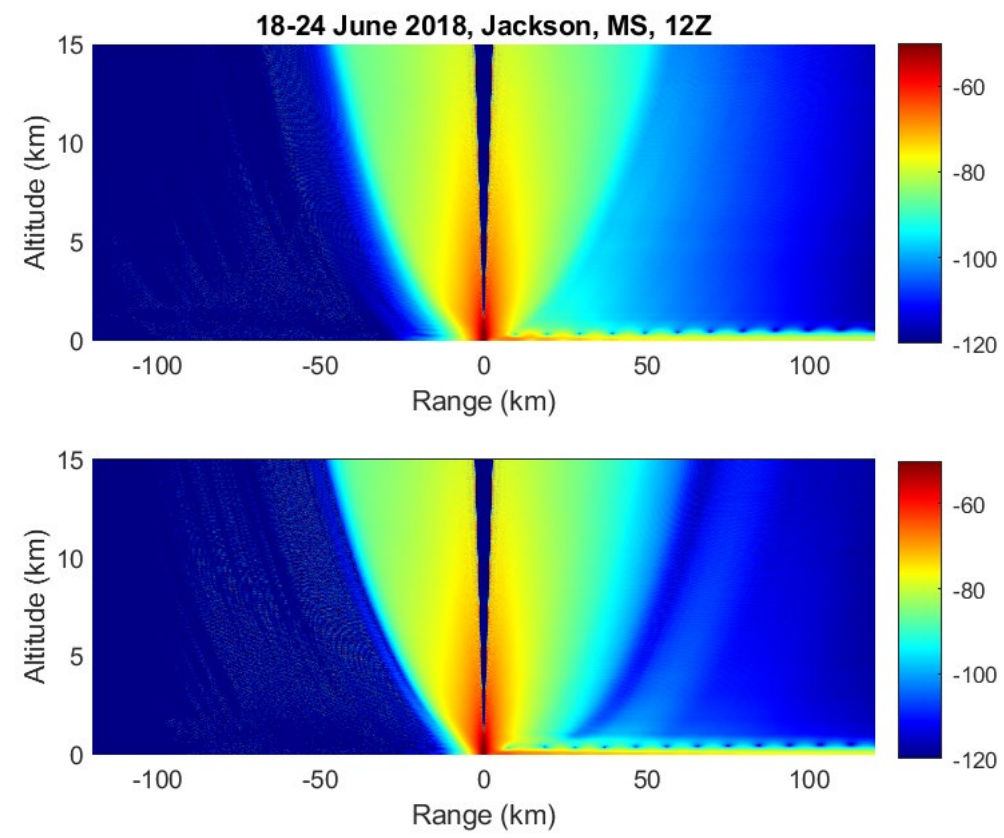
Figure 47. TL versus distance, 19-25 September 2018, 12Z, Danmarkshavn, Greenland. Thick black line represents the mean profile for the week, all other lines represent individual days. Top plot shows propagation to the west (left) and east (right). Bottom plot shows propagation to the south (left) and north (right). Frequency is $4 \mathrm{~Hz}$.
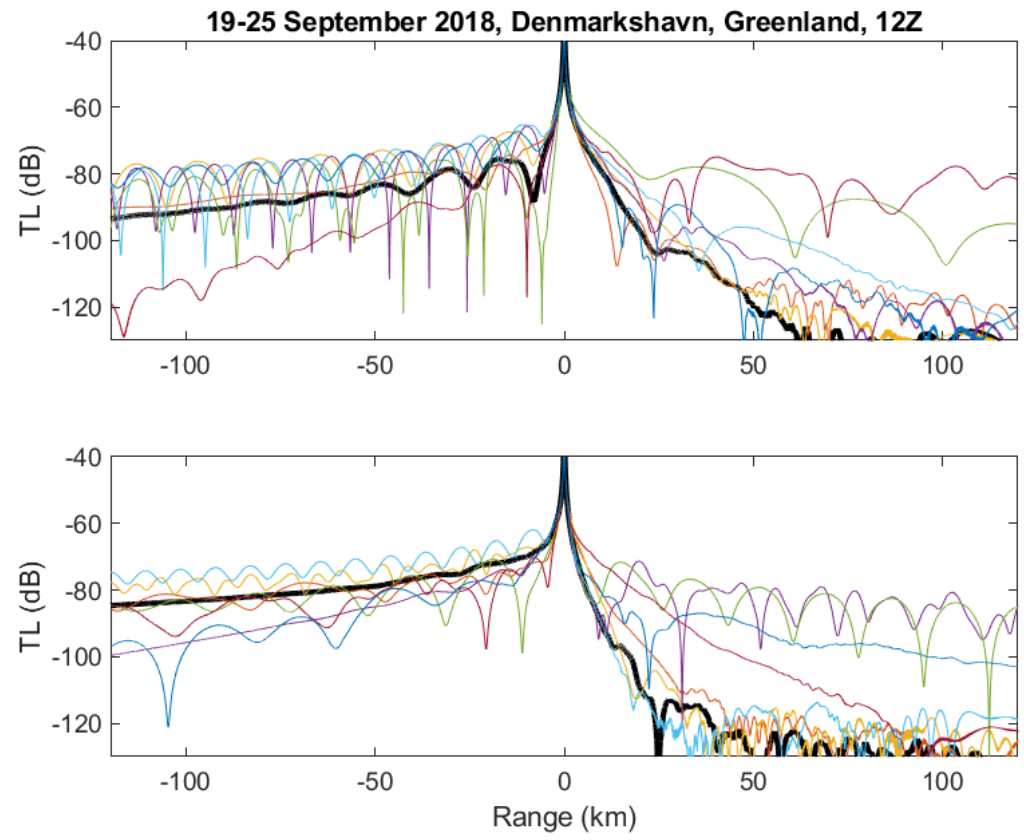

Figure 48. TL as a function of range and altitude, mean profile for the 19-25 September 2018 time period, 12Z, in Danmarkshavn, Greenland. Top plot shows propagation to the west (left) and east (right). Bottom plot shows propagation to the south (left) and north (right).

Frequency is $4 \mathrm{~Hz}$.
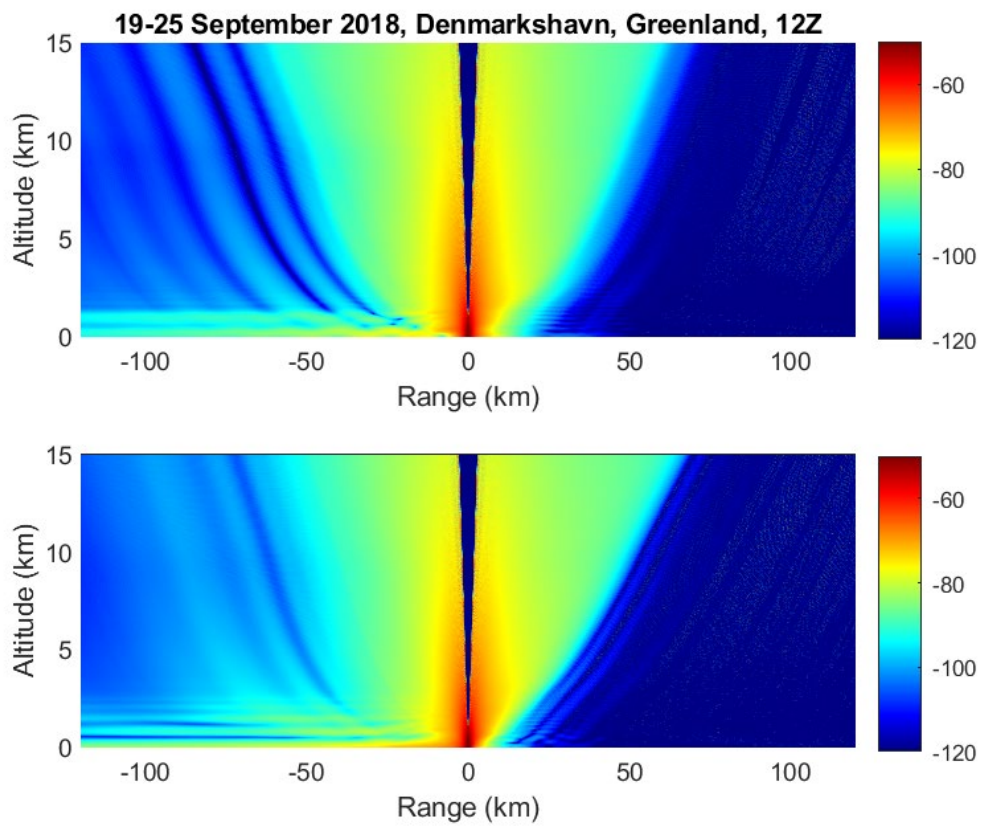
Figure 49. TL versus distance, 19-25 September 2018, 12Z, Fairbanks, AK. Thick black line represents the mean profile for the week, all other lines represent individual days. Top plot shows propagation to the west (left) and east (right). Bottom plot shows propagation to the south (left) and north (right). Frequency is $4 \mathrm{~Hz}$.
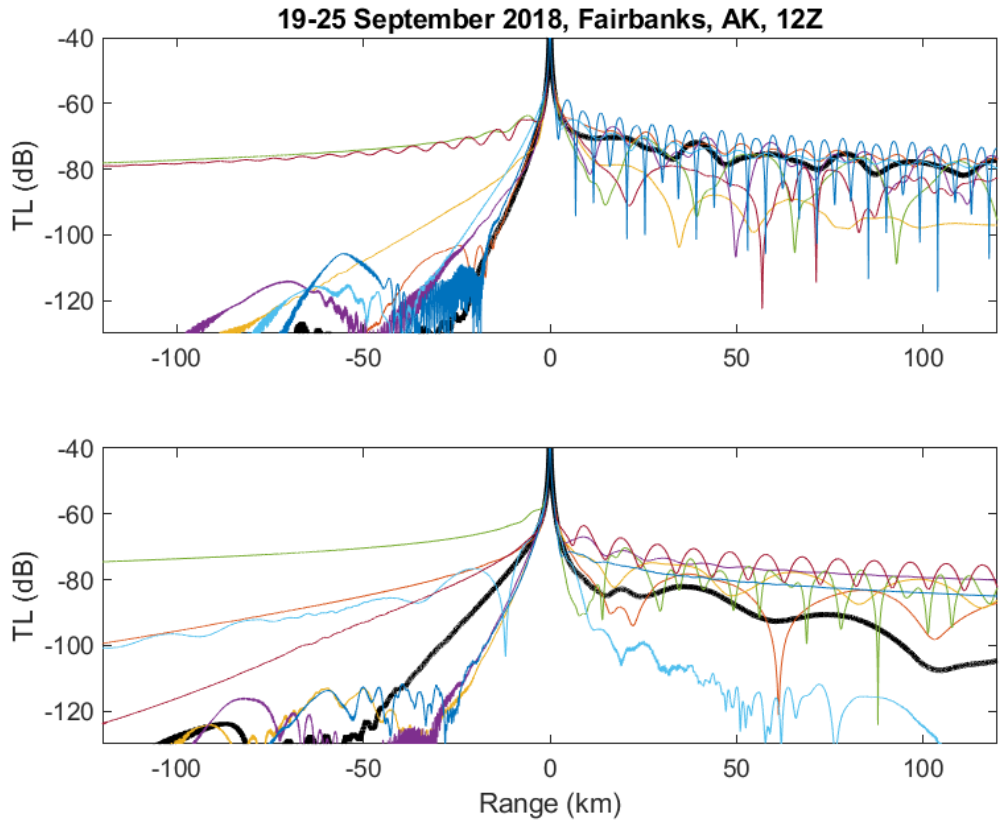

Figure 50. TL as a function of range and altitude, mean profile for the 19-25 September 2018 time period, 12Z, in Fairbanks, AK. Top plot shows propagation to the west (left) and east (right). Bottom plot shows propagation to the south (left) and north (right). Frequency is 4 $\mathrm{Hz}$.
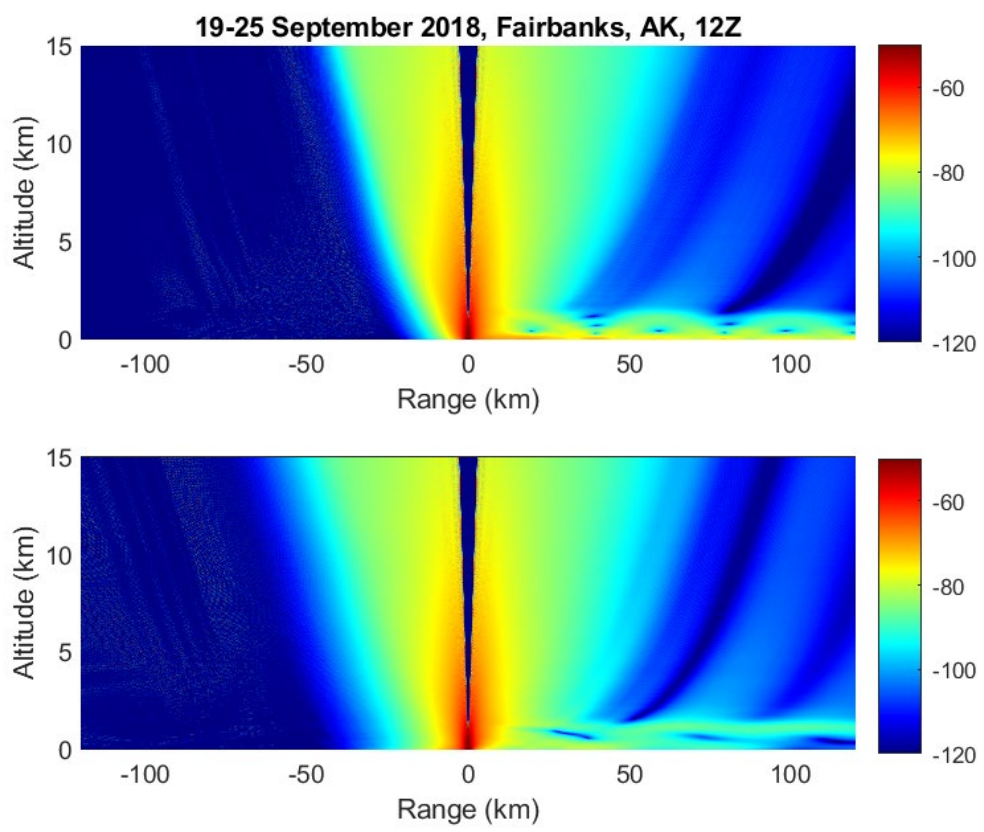
Figure 51. TL versus distance, 19-25 September 2018, 12Z, Jackson, MS. Thick black line represents the mean profile for the week, all other lines represent individual days. Top plot shows propagation to the west (left) and east (right). Bottom plot shows propagation to the south (left) and north (right). Frequency is $4 \mathrm{~Hz}$.
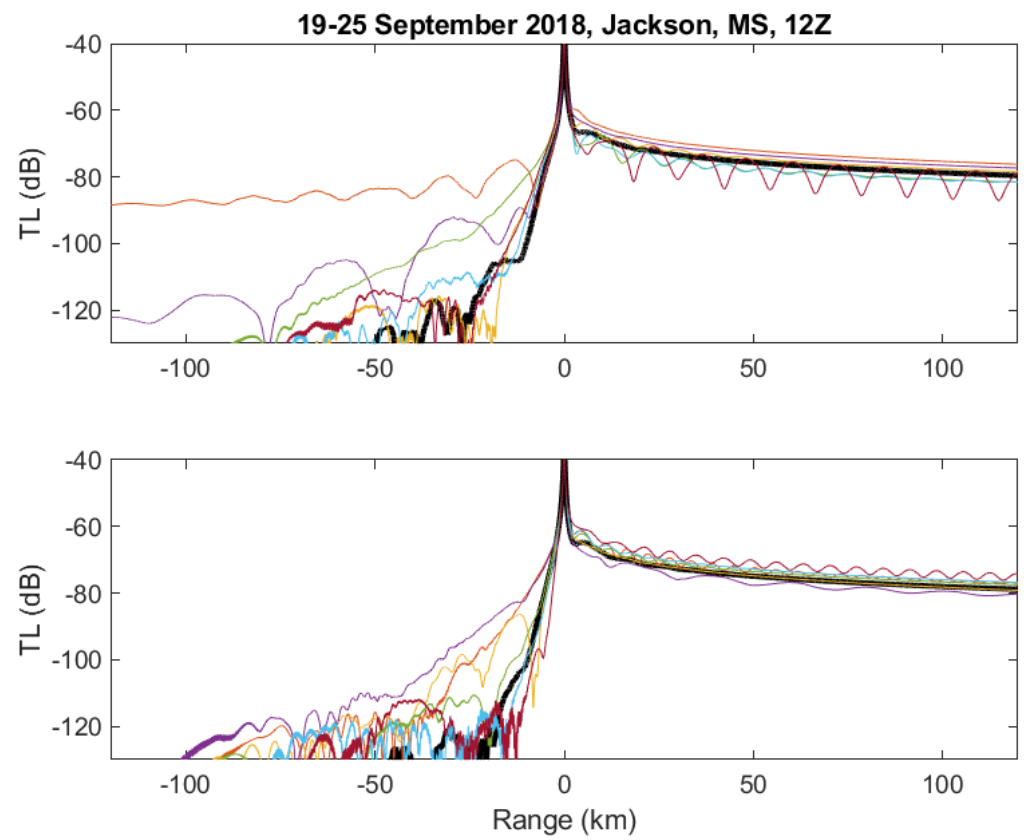

Figure 52. TL as a function of range and altitude, mean profile for the 19-25 September 2018 time period, 12Z, in Jackson, MS. Top plot shows propagation to the west (left) and east (right). Bottom plot shows propagation to the south (left) and north (right). Frequency is $4 \mathrm{~Hz}$.
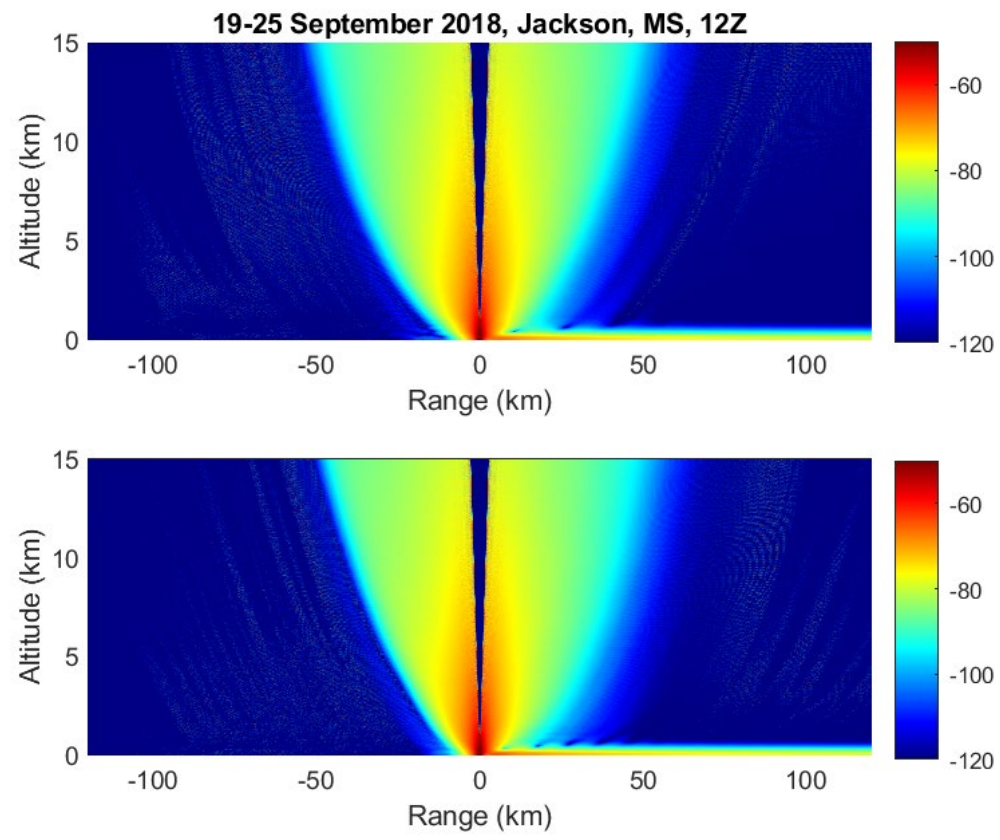
Figure 53. TL versus distance, 18-24 December 2018, 12Z, Danmarkshavn, Greenland. Thick black line represents the mean profile for the week, all other lines represent individual days. Top plot shows propagation to the west (left) and east (right). Bottom plot shows propagation to the south (left) and north (right). Frequency is $4 \mathrm{~Hz}$.
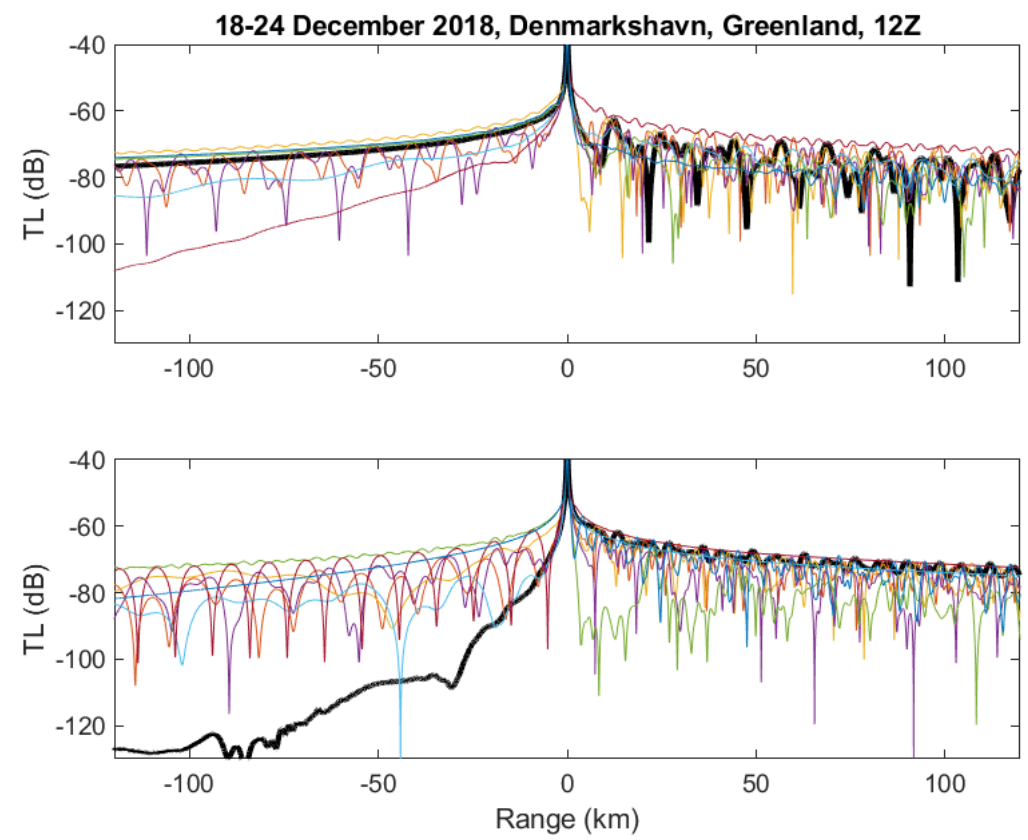

Figure 54. TL as a function of range and altitude, mean profile for the 18-24 December 2018 time period, 12Z, in Danmarkshavn, Greenland. Top plot shows propagation to the west (left) and east (right). Bottom plot shows propagation to the south (left) and north (right). Frequency is $4 \mathrm{~Hz}$.
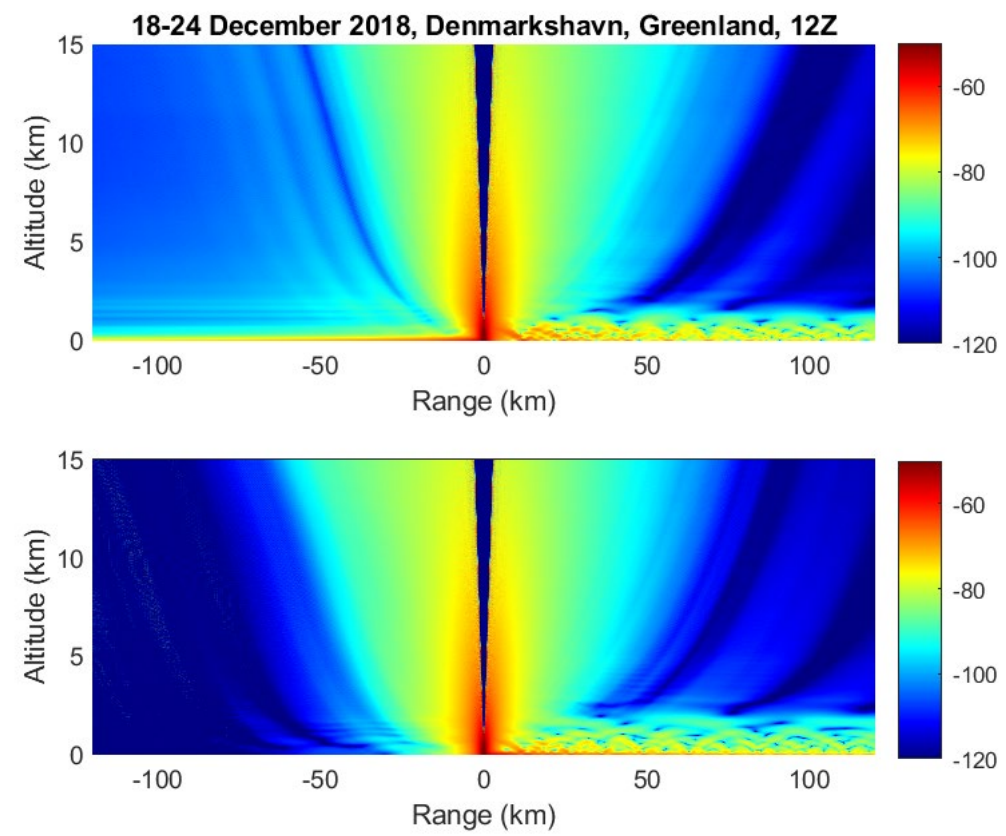
Figure 55. TL versus distance, 18-24 December 2018, 12Z, Fairbanks, AK. Thick black line represents the mean profile for the week, all other lines represent individual days. Top plot shows propagation to the west (left) and east (right). Bottom plot shows propagation to the south (left) and north (right). Frequency is $4 \mathrm{~Hz}$.
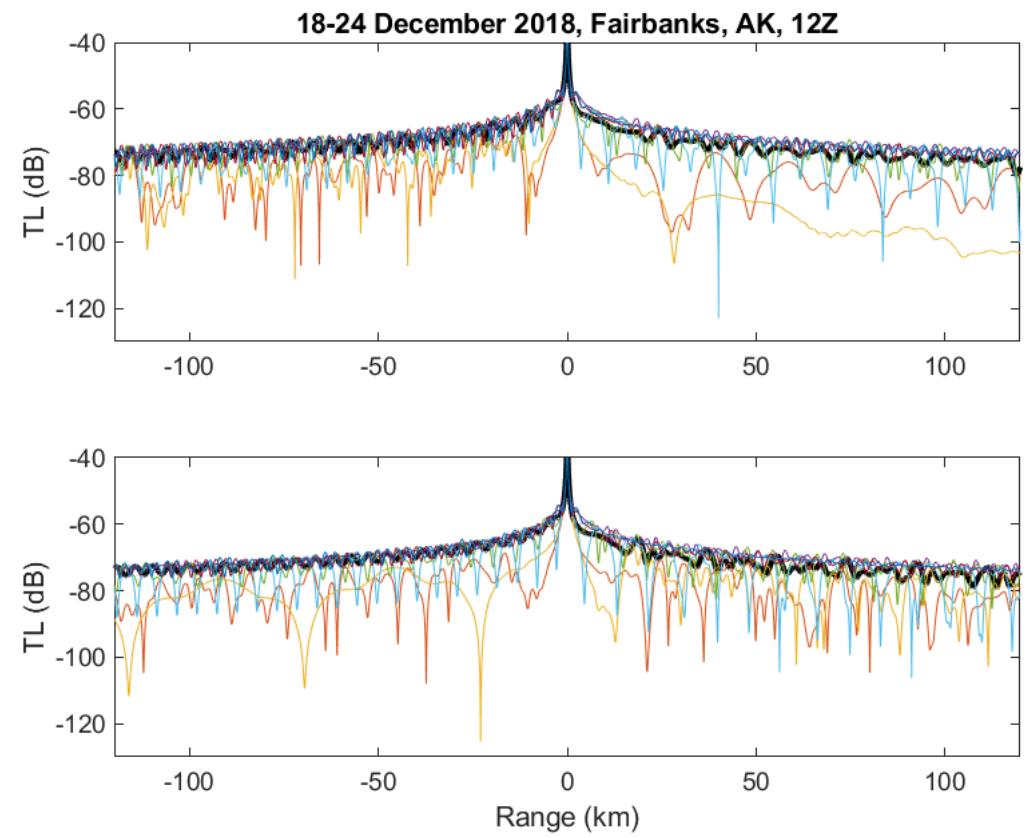

Figure 56. TL as a function of range and altitude, mean profile for the 18-24 December 2018 time period, 12Z, in Fairbanks, AK. Top plot shows propagation to the west (left) and east (right). Bottom plot shows propagation to the south (left) and north (right). Frequency is $4 \mathrm{~Hz}$.
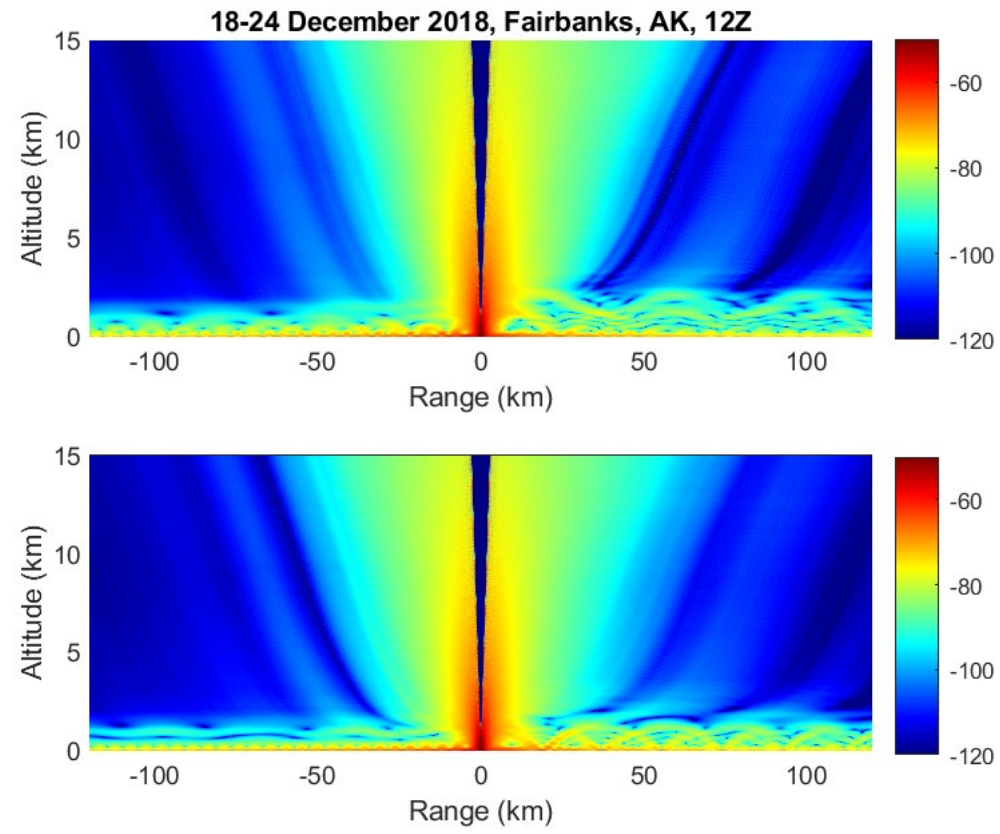
Figure 57. TL versus distance, 18-24 December 2018, 12Z, Jackson, MS. Thick black line represents the mean profile for the week, all other lines represent individual days. Top plot shows propagation to the west (left) and east (right). Bottom plot shows propagation to the south (left) and north (right). Frequency is $4 \mathrm{~Hz}$.
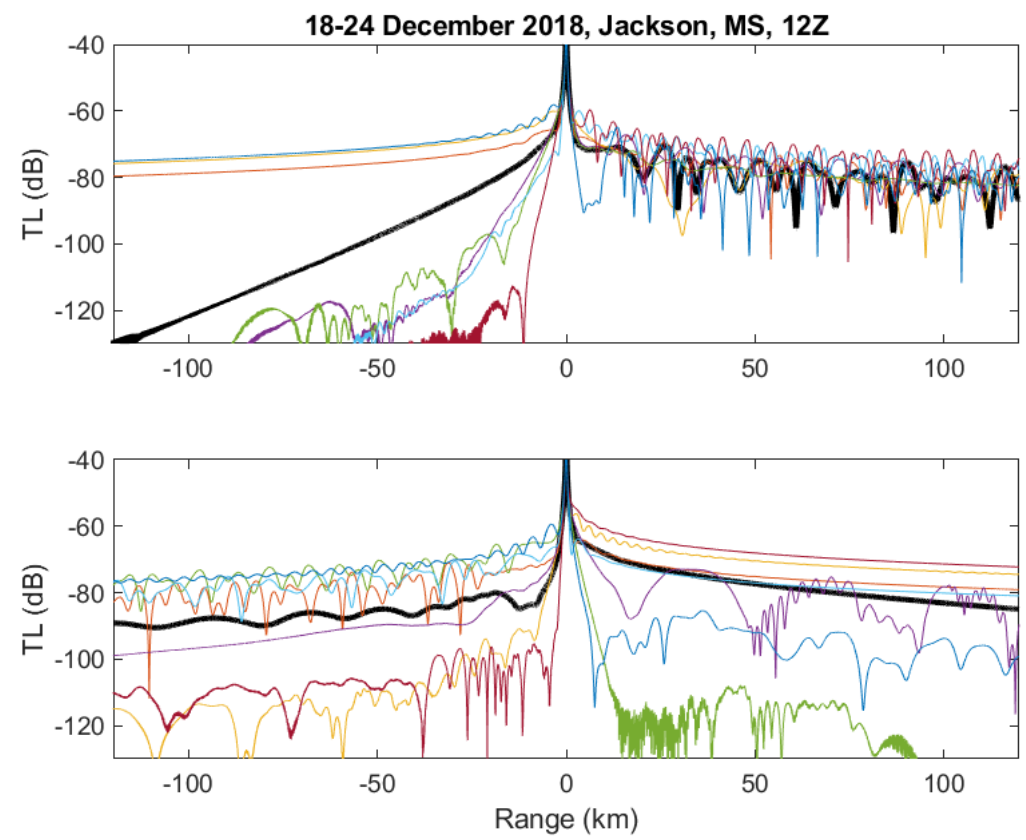

Figure 58. TL as a function of range and altitude, mean profile for the 18-24 December 2018 time period, 12Z, in Jackson, MS. Top plot shows propagation to the west (left) and east (right). Bottom plot shows propagation to the south (left) and north (right). Frequency is $4 \mathrm{~Hz}$.
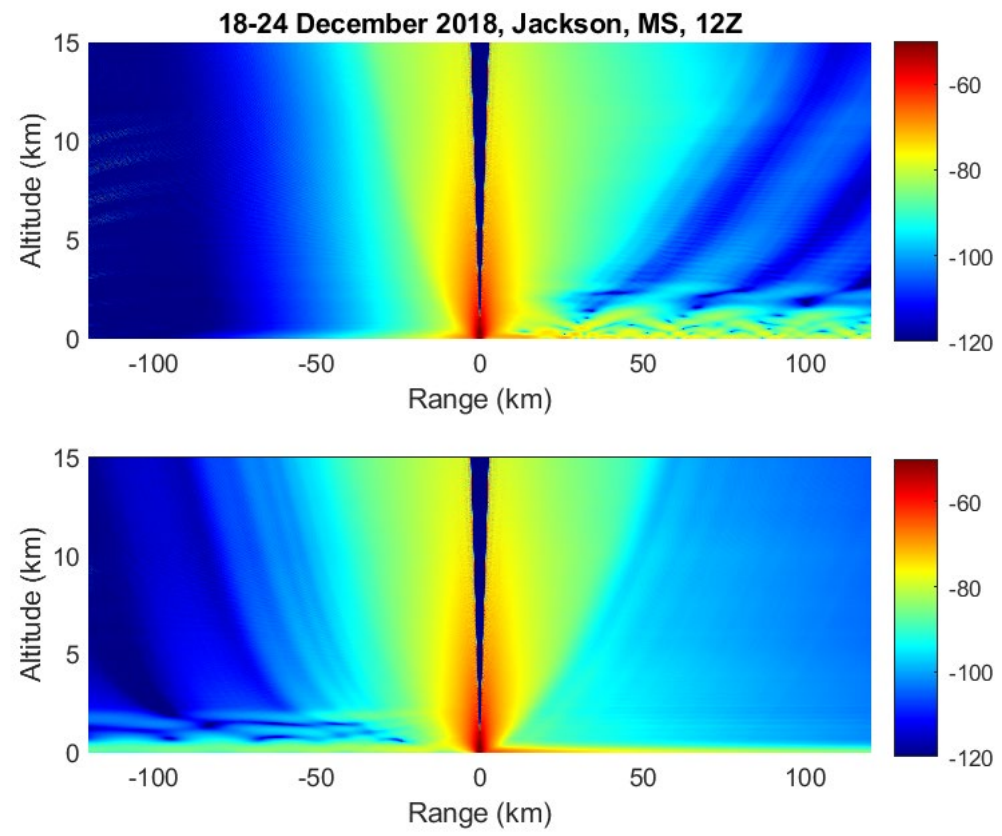


\section{Conclusion}

This project provided a baseline understanding of infrasound propagation in the Arctic, including characteristic horizontal propagation distances, frequency dependencies, and conditions leading to enhanced propagation. This information is useful for devising strategies to monitor natural and man-made phenomena. For example, it may be possible to employ infrasonic systems to remotely sense the upper atmosphere in the Arctic to provide early warning of the polar vortex or to discern industrial and military activities at a long distance.

This project emphasized numerical modeling approaches as an initial step toward improving understanding of the basic phenomenology of infrasonic propagation in the Arctic. Specifically, it combined mesoscale numerical weather forecasts from the PWRF model with advanced acoustic propagation techniques suitable for an atmosphere with high winds.

From an acoustical modeling perspective, this project produced significant advances with regard to parabolic equation modeling of sound propagation in a windy atmosphere. The starting point was a new EWAPE, which is valid for essentially any propagation angle but is rather challenging to solve numerically. From the EWAPE, a new WAPE solution was derived using a Padé $(1,1)$ approximation. Unlike conventional NAPEs, which are widely used at present and suitable only for propagation angles up to $15^{\circ}-20^{\circ}$, the new WAPE is suitable for propagation angles up to roughly $35^{\circ}-40^{\circ}$. Furthermore, the WAPE does not involve the effective sound speed approximation. It is valid for low Mach numbers and wellsuited to many applications involving refraction and scattering from elevated layers in a windy atmosphere. Conveniently, the new WAPE can be implemented with very simple changes to conventional CrankNicholson NAPE codes and is no more computationally intensive.

The new WAPE was combined with PWRF modeling of the Arctic atmosphere. We focused in this report on two examples that illustrate particularly interesting propagation effects, namely a polar low ("Arctic hurricane") in the East Siberian Sea and a katabatic wind in northern Greenland. For the polar low, some interesting interactions with the stratosphere were found, which could possibly be used to provide early warning of strong stratospheric warming events (i.e., the polar vortex). The katabatic wind resulted in a very strong low-level duct that, when 
combined with a highly reflective icy ground surface, would lead to efficient long-distance propagation.

A comparative study was also conducted of propagation in four different seasonal weather conditions (around the summer and winter solstices, and the spring and fall equinoxes) at three different characteristic locations: Fairbanks, Alaska (an inland Arctic environment); Danmarkshavn, Greenland (a coastal Arctic environment); and Jackson, Mississippi (a temperate environment). The results showed that, at the Arctic locations, a persistently strong temperature inversion during a week in December was the primary cause of strong surface ducting and long-range propagation that is evident only intermittently at other times of year and at temperate latitudes. In contrast, the wintertime propagation characteristics in Jackson appear to be heavily impacted by the jet stream.

This project casts light on how the differing atmospheric and land surface conditions at northern latitudes impact infrasound propagation in comparison to temperate latitudes. In particular, the simulations predict the existence of unusually strong and persistent long-range propagation effects. With these insights, productive experiments can be conducted to validate the predictions. The modeling advances also lay a foundation for applied research involving assimilation of numerical weather forecast data into operational infrasound predictions or, conversely, for assimilation of infrasound data into numerical weather forecasts. 


\section{References}

Andreas, E. L., 1988. "Estimating Cn2 over Snow and Sea Ice from Meteorological Data." Journal of the Optical Society of America A 5 (4): 481-95.

Asming, V. E., S. V. Baranov, A. N. Vinogradov, and A. V. Fedorov. 2016. "Using an Infrasonic Method to Monitor the Destruction of Glaciers in Arctic Conditions." Acoustical Physics 62: 583-92.

Blanc-Benon, P., L. Dallois, and D. Juvé. 2001. "Long Range Sound Propagation in a Turbulent Atmosphere within the Parabolic Approximation." Acta Acustica united with Acustica 87 (6): 659-69.

Bromwich, D. H., A. B. Wilson, L. Bai, Z. Liu, M. Barlage, C. Shih, S. Maldonado, et al. 2018. "The Arctic System Reanalysis, Version 2." Bulletin of the American Meteorological Society 99: 805-28.

Butler, A. H., J. P. Sjoberg, D. J. Seidel, and K. H. Rosenlof. 2017. "A Sudden Stratospheric Warming Compendium.” Earth System Science Data 9: 63-76. https://doi.org/10.5194/essd-9-63-2017.

Center for Strategic and International Studies, 2020. Russia's Military Presence in the Arctic. https://www.csis.org/features/ice-curtain-russias-arctic-military-presence.

Collins, M. D. 1991. "Higher-Order Padé Approximations for Accurate and Stable Elastic Parabolic Equations with Application to Interface Wave Propagation.” Journal of the Acoustical Society of America 89: 1050-57.

Collins, M. D. 1993. “A Split-Step Padé Solution for the Parabolic Equation Method.” Journal of the Acoustical Society of America 93(4): 1736-42.

Coy, L., and S. Pawson. 2015. "The Major Stratospheric Sudden Warming of January 2013: Analyses and Forecasts in the GEOS-5 Data Assimilation System." Monthly Weather Review 143(2): 491-510.

Drob, D. P., J. M. Picone, and M. Garcés. 2003. "Global Morphology of Infrasound Propagation.” Journal of Geophysical Research: Atmospheres 108 (D21).

Farmer, D. M., and Y. Xie. 1989. "The Sound Generated by Propagating Cracks in Sea Ice. Journal of the Acoustical Society of America 85 (4): 1489-1500.

Fornberg, B. 1988. "Generation of Finite Difference Formulas on Arbitrarily Spaced Grids.” Mathematics of Computation 51 (184): 699-706.

Gavrilov, A. N., and P. N. Mikhalevsky. 2006. "Low-Frequency Acoustic Propagation Loss in the Arctic Ocean: Results of the Arctic Climate Observations Using Underwater Sound Experiment.” Journal of the Acoustical Society of America 119 (6): 3694706. https://doi.org/10.1121/1.2195255.

Gibbons, S. J., and F. Ringdal. 2010. "Databases for Studies of Infrasound Propagation in the European Arctic.” EGU General Assembly Conference Abstracts 12: 4661.

Abstract only. 
Gibson, R. G., and D. P. Drob. 2005. "Infrasound Propagation Calculation Techniques Using Synoptic and Mesoscale Atmospheric Specifications." In Proceedings of the 27th Seismic Research Review: Ground-Based Nuclear Explosion Monitoring Technologies, Tech. Rep. LA-UR-05-6407: 828-37. Los Alamos, NM: Los Alamos National Laboratory.

Gilbert K. E., and M. J. White. 1989. "Application of the Parabolic Equation to Sound Propagation in a Refracting Atmosphere." Journal of the Acoustical Society of America 85 (2):630-37.

Gilbert, K. E., and X. Di. 1993. “A Fast Green's Function Method for One-Way Sound Propagation in the Atmosphere.” Journal of the Acoustical Society of America 94 (4): 2343-52.

Guest, P., P. O. G. Persson, S. Wang, M. Jordan, Y. Jin, B. Blomquist, and C. Fairall. 2018. "Low-Level Baroclinic Jets over the New Arctic Ocean." Journal of Geophysical Research: Oceans 123: 4074-91.

Hines, K. M., and D. Bromwich. 2008. "Development and Testing of Polar Weather Research and Forecasting (WRF) Model. Part I: Greenland Ice Sheet Meteorology." Monthly Weather Review 136(6): 1971-89.

Hines, K. M., D. H. Bromwich, L. Bai, C. M. Bitz, J. G. Powers and K. W. Manning. 2015. "Sea Ice Enhancements to Polar WRF." Monthly Weather Review 143 (6): 236385.

Jakobson, L., T. Vihma, E. Jakobson, T. Palo, A. Männik, and J. Jaagus. 2013. "Low-Level Jet Characteristics over the Arctic Ocean in Spring and Summer." Atmospheric Chemistry and Physics 13: 11089-99.

Lingevitch, J. F., M. D. Collins, D. K. Dacol, D. P. Drob, J. C. W. Rogers, and W. L. Siegmann. 2002. "A Wide Angle and High Mach Number Parabolic Equation. Journal of the Acoustical Society of America 111 (2): 729-34.

McKenna, M. H., B. W. Stump, and C. Hayward. 2008. "Effect of Time-Varying Tropospheric Models on Near-Regional and Regional Infrasound Propagation as Constrained by Observational Data." Journal of Geophysical Research: Atmospheres 113: D11111.

McKenna, M., R. Gibson, B. Walker, J. McKenna, N. Winslow, and A. Kofford. 2012. "Topographic Effects on Infrasound Propagation." Journal of the Acoustical Society of America 131 (1): 35-46.

National Center for Atmospheric Research/University Corporation for Atmospheric Research, and Polar Meteorology Group/Byrd Polar and Climate Research Center/The Ohio State University. 2017. "Arctic System Reanalysis version 2." Research Data Archive at the National Center for Atmospheric Research, Computational and Information Systems Laboratory. https://rda.ucar.edu/datasets/ds631.1/.

Okada, Y., K. Yoshihisa, and K. Tatsuda. 2016. “Annual Fluctuation of Atmospheric Absorption of Sound in Various World Regions." Acoustical Science and Technology 37 (2): 66-74. 
Olson, J. V., and C. A. Szuberla. 2005. "Distribution of Wave Packet Sizes in Microbarom Wave Trains Observed in Alaska." Journal of the Acoustical Society of America 117 (3): 1032-37.

Ostashev, V. E., D. Juvé, and P. Blanc-Benon. 1997. "Derivation of a Wide-Angle Parabolic Equation for Sound Waves in Inhomogeneous Moving Media." Acta Acustica United with Acustica 83 (3): 455-60.

Ostashev, V. E., and D. K. Wilson. 2015. Acoustics in Moving Inhomogeneous Media. Boca Raton, FL: CRC Press.

Ostashev, V. E., D. K. Wilson, and M. B. Muhlestein. 2020. "Wave and Extra-Wide-Angle Parabolic Equations for Sound Propagation in a Moving Atmosphere." Journal of the Acoustical Society of America 147: 3969-84. doi:10.1121/10.0001397.

Ostashev, V. E., M. B. Muhlestein, and D. K. Wilson. 2019. "Extra-Wide-Angle Parabolic Equations in Motionless and Moving Media." Journal of the Acoustical Society of America 145: 1031-47. doi:10.1121/1.5091011.

Ostashev V. E., P. Blanc-Benon, D. Juvé, and L. Dallois. 2002. "Wide Angle Parabolic Equation for Sound Waves in a Refractive, Turbulent Atmosphere." Proceedings of the 1oth International Symposium on Long Range Sound Propagation: 6272. Grenoble, France.

Pierce, A. D. 1981. Acoustics: An Introduction to Its Physical Principles and Applications. New York: McGraw-Hill.

Ringdal, F., T. Kværna, S. J. Gibbons, S. Mykkeltveit, and J. Schweitzer. 2010. Basic Research on Seismic and Infrasonic Monitoring of the European Arctic. Kjeller, Norway: NORSAR.

Saad, Y., and D. Lee. 1986. "A New Algorithm for Solving the Wide Angle Wave Equation.” Research Report YALEU/DCS/RR-485.

Salomons, E. 2001. Computational Atmospheric Acoustics. Dordrecht: Kluwer Academic.

Simmonds, I., and I. Rudeva. 2012. "The Great Arctic Cyclone of August 2012." Geophysical Research Letters 39: L23709.

Szuberla, C. A., and J. V. Olson. 2004. "Uncertainties Associated with Parameter Estimation in Atmospheric Infrasound Arrays. Journal of the Acoustical Society of America 115 (1): 253-58.

van As, D., T. Fausto, K. Steffen, A. Ahlstrøm, S. Andersen, M. Andersen, J. Box, et al. 2014. "Katabatic Winds and Piteraq Storms: Observations from the Greenland Ice Sheet. Geologic Survey of Denmark and Greenland 31: 83-86.

van den Broeke, M. R., P.G. Duynkerke, and J. Oerlemans. 1994. "The Observed Katabatic Flow at the Edge of the Greenland Ice Sheet during GIMEX-91." Global and Planetary Change 9 (1-2): 3-15.

Waxler, R., C. L. Talmadge, S. Dravida, and K. E. Gilbert. 2006. “The Near-Ground Structure of the Nocturnal Sound Field. Journal of the Acoustical Society of America 119 (1): 86-95. 
West, M., K. Gilber, and R. A. Sack. 1992. "A Tutorial on the Parabolic Equation (PE) Model Used for Long Range Sound Propagation in the Atmosphere." Applied Accoustics 37 (1): 31-49.

Wilson, A. B., D. H. Bromwich, and K. M. Hines. 2011. "Evaluation of Polar WRF Forecasts on the Arctic System Reanalysis Domain: Surface and Upper Air Analysis." Journal of Geophysical Research 116: D11112. doi: 10.1029/2010JD015013.

Wilson, C. R., J. V. Olson, and H. C. Stenbaek-Nielsen. 2005. "High Trace-Velocity Infrasound from Pulsating Auroras at Fairbanks, Alaska. Geophysical Research Letters 32: L14810.

Wilson, D. K., D. J. Breton, L. E. Waldrop, D. R. Glaser, R. E. Alter, C. R. Hart, W. M. Barnes, et al. 2021. Signal Propagation Modeling in Complex, ThreeDimensional Environments (SPRUCE). ERDC/CRREL TR-21-6. Hanover, NH: U.S. Army Engineer Research and Development Center.

Wilson, D. K., D. J. Breton, R. E. Alter, L. E. Waldrop, W. M. Barnes, M. B. Muhlestein, V. E. Ostashev. 2018. "Modeling RF and Acoustic Signal Propagation in Complex Environments.” Ground/Air Multisensor Interoperability, Integration, and Networking for Persistent ISR IX, SPIE Defense and Commercial Sensing, Orlando, 15-19 Apr. 2018, paper 1063519.

Wilson, D. K., and D. W. Thomson. 1991. "Natural Temporal Variability of Atmospheric Acoustic Absorption Coefficients. Applied Acoustics 34 (2): 111-21.

Wilson, D.K., J. M. Noble, and M. A. Coleman. 2003. "Sound Propagation in the Nocturnal Boundary Layer.” Journal of the Atmospheric Sciences 60 (20): 247386.

Wilson, D. K., and K. K. Yamamoto. 2014. Environmental Awareness for Sensor and Emitter Employment (EASEE): Software Design Version 2. ERDC/CRREL TR14-27. Hanover, NH: U.S. Army Engineer Research and Development Center.

Wilson, D. K., M. B. Muhlestein, V. E. Ostashev, M. J. Shaw, M. E. Swearingen, and S. L. McComas. 2019. "Solution of Wide-Angle Parabolic Equations for Long-Range Sound Propagation in a Moving Medium." Proceedings of the International Congress on Acoustics. Aachen, Germany.

Yevick, D., and D. J. Thomson. 200o. "Complex Padé Approximants for Wide-Angle Acoustic Propagators.” Journal of the Acoustical Society of America 108 (6): 2784-90. 


\section{Acronyms and Abbreviations}

ASR

ARW

COAMPS

CPC

CRREL

DoD

EASEE

ERDC

ESSA

EUCOM

EWAPE

GALWEM

GFPE

$\mathrm{Hz}$

ICA

K

LLJ

NAPE

NetCDF

NOAA

NORAD

NORTHCOM
Arctic System Reanalysis

Advanced Research WRF

Coupled Ocean/Atmosphere Mesoscale Prediction System

Climate Prediction Center

U.S. Army Cold Regions Research and Engineering Laboratory

U.S. Department of Defense

Environmental Awareness for Sensor and Emitter Employment

U.S. Army Engineer Research and Development Center

effective sound-speed approximation

U.S. European Command

extra-wide-angle parabolic equation

Global Air-Land Weather Exploitation Model

Green's function parabolic equation

Hertz

International Congress on Acoustics

Kelvin

low-level jet

narrow-angle parabolic equation

Network Common Data Form

National Oceanic and Atmospheric Administration

North American Aerospace Defense Command

U.S. Northern Command 


$\begin{array}{ll}\text { PE } & \text { parabolic equation } \\ \text { PWRF } & \text { Polar WRF } \\ \text { RF } & \text { radio frequency } \\ \text { SSW } & \text { sudden stratospheric warming } \\ \text { TL } & \text { transmission loss } \\ \text { UTC } & \text { Universal Coordinated Time } \\ \text { WAPE } & \text { wide-angle parabolic equation } \\ \text { WRF } & \text { Weather Research and Forecasting model }\end{array}$




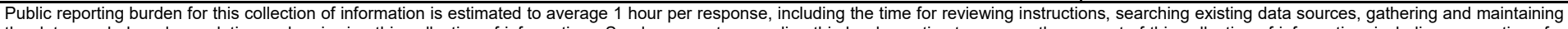

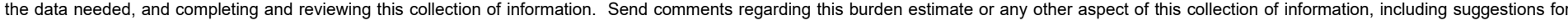

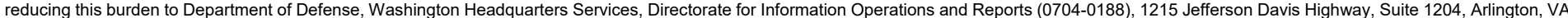

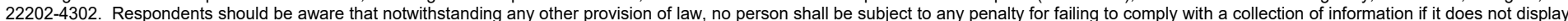
a currently valid OMB control number. PLEASE DO NOT RETURN YOUR FORM TO THE ABOVE ADDRESS.

\section{REPORT DATE (DD-MM-YYYY) \\ December 2021 \\ 2. REPORT TYPE \\ Technical Report / Final}

\section{TITLE AND SUBTITLE}

Infrasound Propagation in the Arctic

3. DATES COVERED (From - To) FY18-FY19

5a. CONTRACT NUMBER

5b. GRANT NUMBER

5c. PROGRAM ELEMENT NUMBER

0601102A

\section{AUTHOR(S)}

D. Keith Wilson, Vladimir E. Ostashev, Michael J. Shaw, Michael B. Muhlestein, John W.

Weatherly, Michelle E. Swearingen, and Sarah L. McComas

5d. PROJECT NUMBER

$\mathrm{T} 22$

5e. TASK NUMBER

02

5f. WORK UNIT NUMBER

\section{PERFORMING ORGANIZATION REPORT}

ERDC-TR-21-22
U.S. Army Engineer Research and Development Center (ERDC)

Cold Regions Research and Engineering Laboratory (CRREL)

72 Lyme Road

Hanover, NH 03755-1290

Construction Engineering Research Laboratory (CERL)

U.S. Army Engineer Research and Development Center (ERDC)

2902 Newmark Drive

Champaign, IL 61822

Geotechnical and Structures Laboratory (GSL)

U.S. Army Engineer Research and Development Center (ERDC)

3909 Halls Ferry Road

Vicksburg, MS 39180-6199

\section{SPONSORING / MONITORING AGENCY NAME(S) AND ADDRESS(ES)}

U.S. Army Engineer Research and Development Center (ERDC)

Cold Regions Research and Engineering Laboratory (CRREL)

72 Lyme Road

Hanover, NH 03755-1290

\section{DISTRIBUTION / AVAILABILITY STATEMENT}

Approved for public release; distribution is unlimited.

\section{SUPPLEMENTARY NOTES}

\section{ABSTRACT}

This report summarizes results of the basic research project "Infrasound Propagation in the Arctic." The scientific objective of this project was to provide a baseline understanding of the characteristic horizontal propagation distances, frequency dependencies, and conditions leading to enhanced propagation of infrasound in the Arctic region. The approach emphasized theory and numerical modeling as an initial step toward improving understanding of the basic phenomenology, and thus lay the foundation for productive experiments in the future. The modeling approach combined mesoscale numerical weather forecasts from the Polar Weather Research and Forecasting model with advanced acoustic propagation calculations. The project produced significant advances with regard to parabolic equation modeling of sound propagation in a windy atmosphere. For the polar low, interesting interactions with the stratosphere were found, which could possibly be used to provide early warning of strong stratospheric warming events (i.e., the polar vortex). The katabatic wind resulted in a very strong low-level duct, which, when combined with a highly reflective icy ground surface, leads to efficient long-distance propagation. This information is useful in devising strategies for positioning sensors to monitor environmental phenomena and human activities.

\section{SUBJECT TERMS}

Infrasonic waves, Sound-waves, Arctic regions, Sound--Propagation

\section{SECURITY CLASSIFICATION OF:}

\section{a. REPORT}

Unclassified

\section{b. ABSTRACT}

Unclassified c. THIS PAGE

Unclassified

\section{LIMITATION} OF ABSTRACT

None

\section{NUMBER} OF PAGES

90 19a. NAME OF RESPONSIBLE PERSON

19b. TELEPHONE NUMBER (include area code) 